\title{
Photocatalytic ATRA Reaction Promoted by lodo-Bodipy and Sodium Ascorbate
}

\section{Electronic Supplementary Information}

G. Magagnano, ${ }^{a, b}$ A. Gualandi, ${ }^{* a}$ M. Marchini, ${ }^{a, c}$ L. Mengozzi, ${ }^{a}$ P. Ceroni, ${ }^{* a, c}$ and P. G. Cozzi*a

a. Dipartimento di Chimica "G. Ciamician" ALMA MATER STUDIORUM, Università di Bologna, Via Selmi 2, 40126 Bologna, Italy

b. Institute of Chemical Research of Catalonia (ICIQ), Av. Països Catalans 16, 43007 Tarragona, Spain

c. Centro Inter Universitario per la Conversione Chimica dell'Energia Solare (SOLAR-CHEM), 44121 Ferrara, Italy 
Table of contents:

General methods and materials

Photocatalytic ATRA reaction: optimization

General procedure for the photocatalytic ATRA reaction:

Evidences of radical mechanism

Photophysical measurements

Reaction in presence of triethylamine

References 
General methods. ${ }^{1} \mathrm{H}$ NMR spectra were recorded on Varian Mercury 400 spectrometer. Chemical shifts are reported in ppm from TMS with the solvent resonance as the internal standard (deuterochloroform: $\delta=$ $7.27 \mathrm{ppm}$ ). Data are reported as follows: chemical shift, multiplicity $(\mathrm{s}=$ singlet, $\mathrm{d}=$ duplet, $\mathrm{t}=$ triplet, $\mathrm{q}=$ quartet, $d d=$ double duplet, $d t=$ double triplet, $b s=$ broad signal, $m=$ multiplet $)$, coupling constants $(\mathrm{Hz})$. ${ }^{13} \mathrm{C}$ NMR spectra were recorded on Varian MR400 spectrometer. Chemical shifts are reported in ppm from TMS with the solvent as the internal standard (deuterochloroform: $\delta=77.0 \mathrm{ppm}$ ). The elemental composition of the compounds was determined by using an elemental analyzer (Thermo Scientific, Flash 2000, Organic Elemental Analyzer) by means of the flash combustion technique. Chromatographic purification was done with $240-400$ mesh silica gel. Purification on preparative thin layer chromatography was done on Merck TLC silica gel $60 \mathrm{~F}_{254}$.

All reactions were set up under an argon atmosphere in oven-dried glassware using standard Schlenk techniques. Synthesis grade solvents were used as purchased and the reaction mixtures were degassed by three cycles of freeze-pump-thaw.

Materials. If not otherwise stated, all reactions were carried out in flame dried glassware under nitrogen atmosphere. Anhydrous solvents were supplied by Aldrich in Sureseal ${ }^{\circledR}$ bottles and were used as received avoiding further purification. Bodipy $\mathbf{1 b}$ was synthetized following reported procedure. ${ }^{1}$ Precursor $1,3,5,7-$ tetramethyl-8-phenyl-dipyrromethane was prepared using the procedure reported by Zoli and Cozzi. ${ }^{2}$

Compound $\mathbf{3} \mathbf{b}^{3}$ and $\mathbf{3} \mathbf{d}^{4}$ were prepared according to literature procedures. 


\section{Photocatalytic ATRA reaction: optimization}

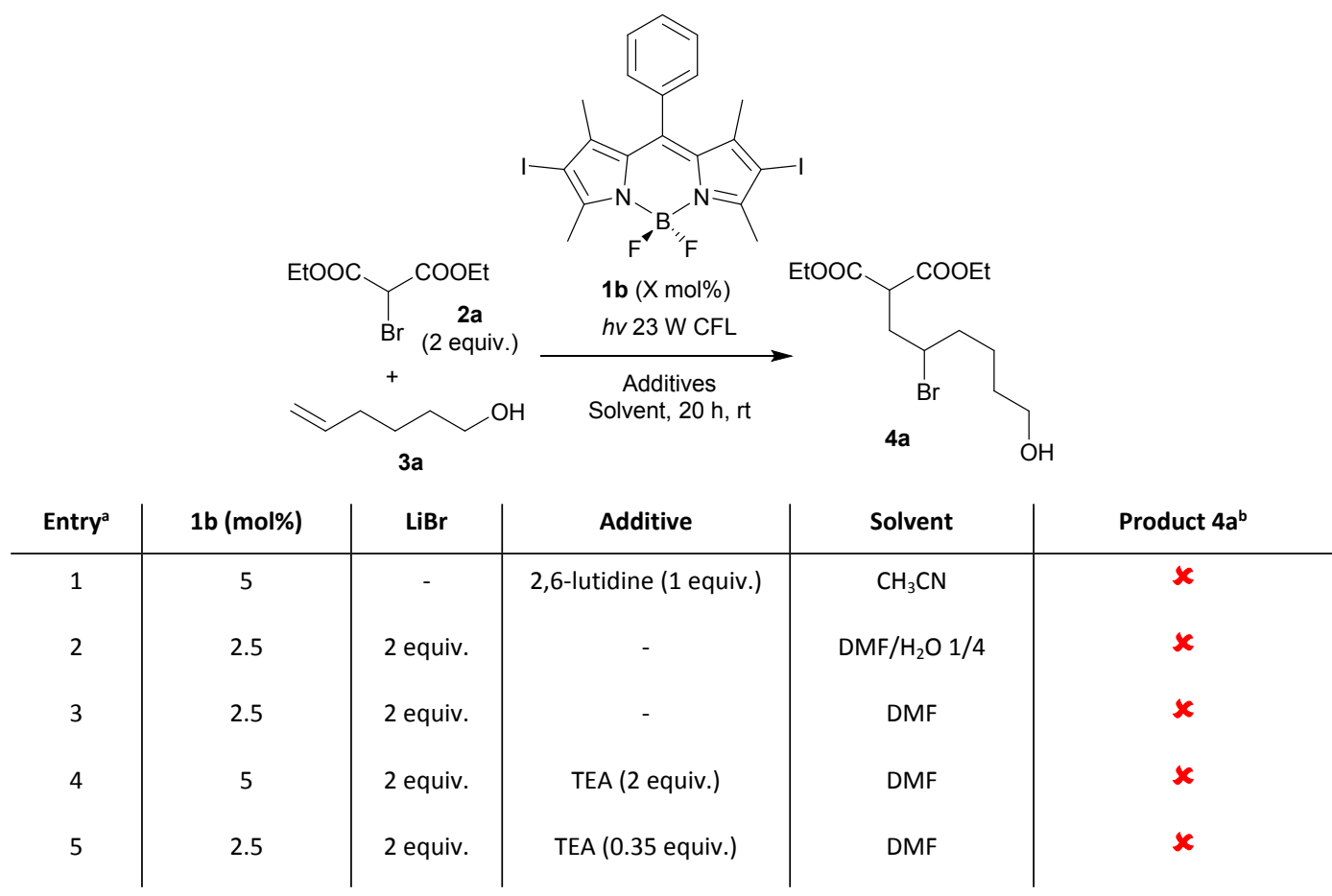

Table S1. a) The reactions were performed using $\mathbf{2 a}(0.2 \mathrm{mmol}), \mathbf{3 a}(0.1 \mathrm{mmol})$ and $\mathbf{1} \mathbf{b}$ in the reported amount, in $200 \mu \mathrm{L}$ of solvent; b) Determined by ${ }^{1} \mathrm{H}-\mathrm{NMR}$ and TLC of the crude.

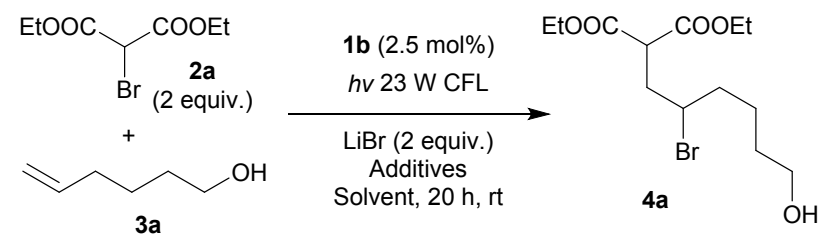

\begin{tabular}{|c|c|c|c|}
\hline Entry ${ }^{a}$ & Additive & Solvent & Product $4 a^{b}$ \\
\hline 1 & TEA ( 0.35 equiv.) & DMF & $\boldsymbol{x}$ \\
\hline 2 & NaAscorbate ( 0.35 equiv.) & $\mathrm{DMF} / \mathrm{H}_{2} \mathrm{O} 4 / 1$ & $\sqrt{(42 \%)}$ \\
\hline 3 & Hantzsch ester ${ }^{c}$ ( 0.35 equiv.) & DMF & $x$ \\
\hline 4 & $\begin{array}{l}\text { NaAscorbate (1 equiv.) } \\
\text { 2,6-lutidine ( } 2 \text { equiv.) }\end{array}$ & $\mathrm{DMF} / \mathrm{H}_{2} \mathrm{O} 4 / 1$ & $x$ \\
\hline 5 & NaAscorbate (1 equiv.) & $\mathrm{DMF} / \mathrm{H}_{2} \mathrm{O} 1 / 1$ & $\sqrt{ }(97 \%)$ \\
\hline
\end{tabular}

Table S2. a) The reactions were performed using $2 \mathrm{a}(0.2 \mathrm{mmol}), 3 \mathrm{a}(0.1 \mathrm{mmol}), \mathrm{LiBr}$ $(0.25 \mathrm{mmol})$, the additives and $\mathbf{1 b}(0.0025 \mathrm{mmol})$ in $200 \mu \mathrm{L}$ of solvent; b) Determined by ${ }^{1} \mathrm{H}-\mathrm{NMR}$ and TLC of the crude, conversion inside brackets; c) Diethyl 1,4-dihydro-2,6dimethyl-3,5-pyridinedicarboxylate. 


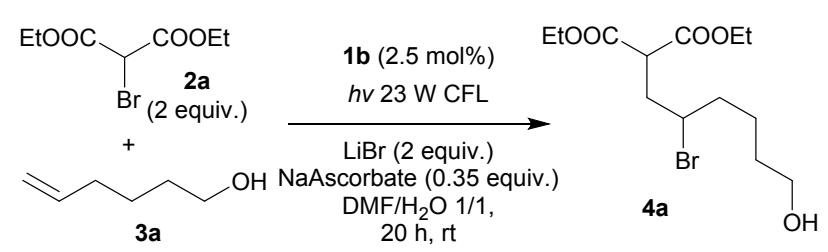

\begin{tabular}{|c|c|c|c|c|c|c|}
\hline Entry ${ }^{a}$ & $1 b$ & $\mathrm{LiBr}$ & NaAscorbate & $h v$ & Air & Product $4 a^{b}$ \\
\hline 1 & Yes & Yes & Yes & Yes & No & $\checkmark(97 \%)$ \\
\hline 2 & No & Yes & Yes & Yes & No & $x$ \\
\hline 3 & Yes & No & Yes & Yes & No & $\checkmark_{(56 \%)}$ \\
\hline 4 & Yes & Yes & No & Yes & No & $x$ \\
\hline 5 & Yes & Yes & Yes & No & No & $x$ \\
\hline 5 & Yes & Yes & Yes & Yes & Yes & $x$ \\
\hline
\end{tabular}

Table S3. a) The reactions were performed using $2 \mathrm{a}(0.2 \mathrm{mmol}), \mathbf{3 a}(0.1 \mathrm{mmol}), \mathrm{LiBr}(0.25 \mathrm{mmol})$, sodium ascorbate $(0.035$ mmol) and $1 \mathbf{b}(0.0025 \mathrm{mmol})$ in $200 \mu \mathrm{L}$ of solvent; b) Determined by ${ }^{1} \mathrm{H}-\mathrm{NMR}$ and TLC of the crude, conversion inside brackets.

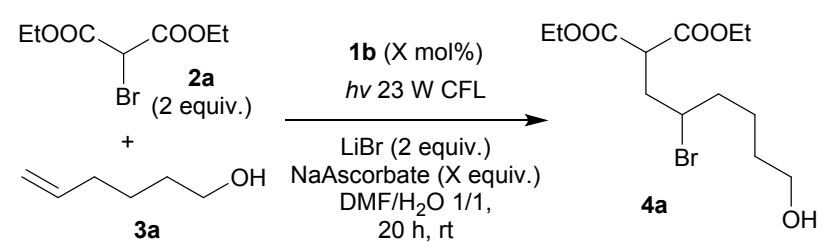

\begin{tabular}{c|c|c|c} 
Entry $^{\mathbf{a}}$ & mol\% 1b & Eq. NaAscorbate & Yield (\%) $^{\mathbf{b}}$ \\
\hline 1 & 2.5 & 1 & 92 \\
2 & 2.5 & 0.35 & 92 \\
3 & 1 & 0.35 & 94
\end{tabular}

Table S4. a) The reactions were performed using $2 \mathrm{a}(0.2 \mathrm{mmol}), 3 \mathrm{a}(0.1 \mathrm{mmol}), \mathrm{LiBr}(0.25$ $\mathrm{mmol}$ ), sodium ascorbate and $\mathbf{1 b}$ in $200 \mu \mathrm{L}$ of solvent; b) After column chromatography.

\section{General procedure for the photocatalytic ATRA reaction:}
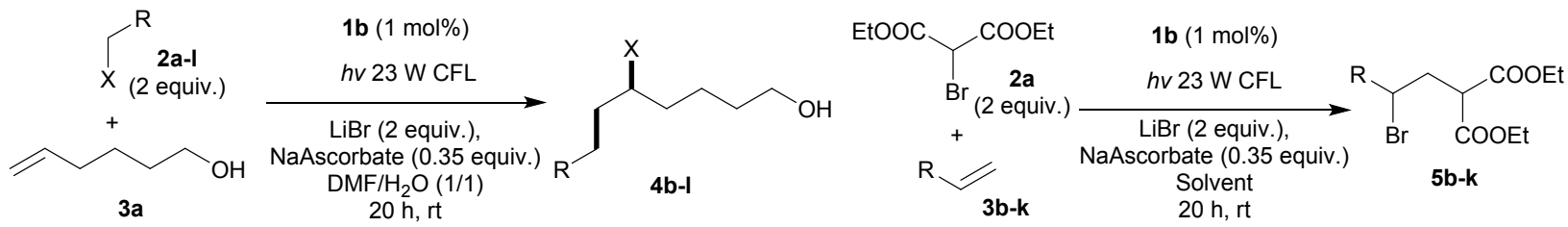

A dry $10 \mathrm{~mL}$ Schlenk tube, equipped with a Rotaflo Stopcock, magnetic stirring bar and an argon supply tube, was charged in order and under argon with the photocatalyst $1 \mathrm{~b}(1 \mathrm{~mol} \%, 0.002 \mathrm{mmol}, 1.2 \mathrm{mg})$, sodium ascorbate ( 0.35 equiv., $0.07 \mathrm{mmol}, 14 \mathrm{mg}$ ), $\mathrm{LiBr}$ ( 2 equiv., $0.4 \mathrm{mmol}, 35 \mathrm{mg}$ ), DMF (200 $\mu \mathrm{L}$ or if 
specified $\mathrm{CH}_{3} \mathrm{CN}$ ), alkyl halide (0.4 mmol, 2 equiv.), olefin ( $0.2 \mathrm{mmol}, 1$ equiv.) and water $(200 \mu \mathrm{L}$, or $\mathrm{MeOH}$ if specified). The reaction mixture was degassed via freeze pump thaw (x3), and the vessel refilled with argon. The reaction mixture was positioned approximately $10 \mathrm{~cm}$ away from the light source $(23 \mathrm{~W} \mathrm{CFL}$ lamp). After vigorous stirring for $20 \mathrm{~h}$, the mixture was transferred in a separator funnel and extracted with AcOEt $(3 \times 5 \mathrm{~mL})$. The combined organic layers were washed with brine $(10 \mathrm{~mL})$, dried over $\mathrm{Na}_{2} \mathrm{SO}_{4}$ and concentrated under reduced pressure to give the crude products. The residue was purified by flash column chromatography $\left(\mathrm{SiO}_{2}\right)$ to afford the title compounds in the stated yields.

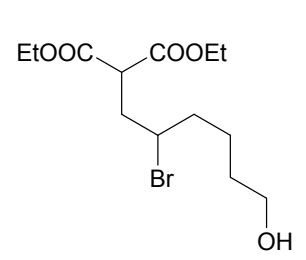

4a: $94 \%$ yield; the reaction was performed following the general procedure using the photocatalyst $\mathbf{1 b}$ (1\% mol, $1.2 \mathrm{mg})$, sodium ascorbate $(0.07 \mathrm{mmol}, 14 \mathrm{mg}), \mathrm{LiBr}(0.4$ mmol, $35 \mathrm{mg})$, DMF (200 $\mu \mathrm{L}), 2 \mathrm{a}(0.4 \mathrm{mmol}, 68 \mu \mathrm{L}), 3 \mathrm{a}(0.2 \mathrm{mmol}, 24 \mu \mathrm{L})$ and $\mathrm{H}_{2} \mathrm{O}(200$ $\mu \mathrm{L})$. Purification by flash column chromatography (7:3 cyclohexane:AcOEt) afforded the title compound $4 \mathrm{a}$ ( $94 \%$ yield, $64 \mathrm{mg}$ ) as a colourless oil. Spectral properties were according to the literature. ${ }^{5}$ HRMS (ESI): calculated for $\mathrm{C}_{13} \mathrm{H}_{23} \mathrm{BrNaO}_{5}{ }^{+}[\mathrm{M}+\mathrm{Na}]^{+}$361.0621, found 361.0625 .

$\overbrace{\text { EtOOC }}^{\mathrm{Br}} \mathrm{CH}_{4}$ 4b: $42 \%$ yield; the reaction was performed following the general procedure using the photocatalyst $1 \mathbf{b}(1 \% \mathrm{~mol}, 1.2 \mathrm{mg})$, sodium ascorbate $(0.07 \mathrm{mmol}, 14 \mathrm{mg}), \mathrm{LiBr}(0.4$ mmol, $35 \mathrm{mg})$, DMF (200 $\mu \mathrm{L}), 2 \mathrm{~b}(0.4 \mathrm{mmol}, 76 \mu \mathrm{L}), 3 \mathbf{a}(0.2 \mathrm{mmol}, 24 \mu \mathrm{L})$ and $\mathrm{H}_{2} \mathrm{O}(200$ $\mu \mathrm{L})$. Purification by flash column chromatography (7:3 cyclohexane:AcOEt) afforded the title compound $\mathbf{4 b}$ ( $42 \%$ yield, $30 \mathrm{mg}$ ) as a colourless oil. Spectral properties were according to the literature. ${ }^{5}$ HRMS (ESI): calculated for $\mathrm{C}_{14} \mathrm{H}_{25} \mathrm{BrNaO}_{5}{ }^{+}[\mathrm{M}+\mathrm{Na}]^{+} 375.0778$, found 375.0782 .<smiles>CCOC(=O)CC(Br)C(Br)C(=O)O</smiles>
4c: $54 \%$ yield; the reaction was performed following the general procedure using the photocatalyst $\mathbf{1 b}(1 \% \mathrm{~mol}, 1.2 \mathrm{mg})$, sodium ascorbate $(0.07 \mathrm{mmol}, 14 \mathrm{mg}), \mathrm{LiBr}(0.4$ mmol, $35 \mathrm{mg})$, DMF (200 $\mu \mathrm{L}), 2 \mathrm{c}(0.4 \mathrm{mmol}, 76 \mu \mathrm{L}), 3 a(0.2 \mathrm{mmol}, 24 \mu \mathrm{L})$ and $\mathrm{H}_{2} \mathrm{O}(200$ $\mu \mathrm{L})$. Purification by flash column chromatography (8:2 cyclohexane:AcOEt) afforded the title compound $4 \mathrm{c}$ ( $54 \%$ yield, $45 \mathrm{mg}$ ) as a colourless oil. Spectral properties were according to the literature. ${ }^{5}$ HRMS (ESI): calculated for $\mathrm{C}_{13} \mathrm{H}_{22} \mathrm{Br}_{2} \mathrm{NaO}_{5}{ }^{+}[\mathrm{M}+\mathrm{Na}]^{+} 438.9726$, found 438.9734 . 
Etooc $\int_{F_{F}^{\prime \prime F}}^{\mathrm{F}_{4}}{ }^{\mathrm{OH}}$

4d: $34 \%$ yield; the reaction was performed following the general procedure using the photocatalyst $1 \mathrm{~b}(1 \% \mathrm{~mol}, 1.2 \mathrm{mg})$, sodium ascorbate $(0.07 \mathrm{mmol}, 14 \mathrm{mg}), \operatorname{LiBr}(0.4$ $\mathrm{mmol}, 35 \mathrm{mg}), \mathrm{DMF}(200 \mu \mathrm{L}), 2 \mathrm{~d}(0.4 \mathrm{mmol}, 76 \mu \mathrm{L}), 3 \mathrm{a}(0.2 \mathrm{mmol}, 24 \mu \mathrm{L})$ and $\mathrm{H}_{2} \mathrm{O}(200$ $\mu \mathrm{L})$. Purification by flash column chromatography (8:2 cyclohexane:AcOEt) afforded the title compound $\mathbf{4 d}$ ( $34 \%$ yield, $21 \mathrm{mg}$ ) as a colourless oil. Spectral properties were according to the literature. ${ }^{6}$ HRMS (ESI): calculated for $\mathrm{C}_{10} \mathrm{H}_{17} \mathrm{BrF}_{2} \mathrm{NaO}_{3}{ }^{+}[\mathrm{M}+\mathrm{Na}]^{+} 325.0221$, found 325.0230 .<smiles>CCOC(=O)CC(Br)C(=O)O</smiles>

4e: $89 \%$ yield; the reaction was performed following the general procedure using the photocatalyst 1b (1\% mol, $1.2 \mathrm{mg})$, sodium ascorbate $(0.07 \mathrm{mmol}, 14 \mathrm{mg}), \mathrm{LiBr}(0.4$ $\mathrm{mmol}, 35 \mathrm{mg})$, DMF $(200 \mu \mathrm{L}), 2 \mathrm{e}(0.4 \mathrm{mmol}, 47 \mu \mathrm{L})$, 3a $(0.2 \mathrm{mmol}, 24 \mu \mathrm{L})$ and $\mathrm{H}_{2} \mathrm{O}(200$ $\mu \mathrm{L})$. Purification by flash column chromatography (7:3 cyclohexane:AcOEt) afforded the title compound $4 \mathrm{e}$ ( $89 \%$ yield, $52 \mathrm{mg}$ ) as a colourless oil. Spectral properties were according to the literature. ${ }^{5}$ HRMS (ESI): calculated for $\mathrm{C}_{10} \mathrm{H}_{18} \mathrm{BrFNaO}_{3}{ }^{+}[\mathrm{M}+\mathrm{Na}]^{+}$307.0316, found 307.0312.<smiles>CCOC(=O)CCC(O)Br</smiles>
4f: $72 \%$ yield; the reaction was performed following the general procedure using the photocatalyst 1b (1\% mol, $1.2 \mathrm{mg})$, sodium ascorbate $(0.07 \mathrm{mmol}, 14 \mathrm{mg}), \mathrm{LiBr}(0.4$ $\mathrm{mmol}, 35 \mathrm{mg})$, DMF $(200 \mu \mathrm{L}), 2 \mathrm{f}(0.4 \mathrm{mmol}, 44 \mu \mathrm{L}), 3 \mathrm{a}(0.2 \mathrm{mmol}, 24 \mu \mathrm{L})$ and $\mathrm{H}_{2} \mathrm{O}(200$ $\mu \mathrm{L})$. Purification by flash column chromatography (7:3 cyclohexane:AcOEt) afforded the title compound $\mathbf{4 f}$ ( $72 \%$ yield, $53 \mathrm{mg}$ ) as a colourless oil. ${ }^{1} \mathrm{H}$ NMR $\left(400 \mathrm{MHz}, \mathrm{CDCl}_{3}, 25^{\circ} \mathrm{C}\right): \delta=4.15(\mathrm{q}, \mathrm{J}=7.1,2 \mathrm{H}), 4.12-4.04(\mathrm{~m}$, $1 H), 3.68(t, J=6.1,2 H), 2.66-2.43(m, 2 H), 2.26-2.15(m, 1 H), 2.14-2.00(m, 1 H), 1.94-1.80(m, 2 H)$, $1.73-1.57(\mathrm{~m}, 4 \mathrm{H}), 1.28(\mathrm{t}, J=7.1,3 \mathrm{H}) ;{ }^{13} \mathrm{C}$ NMR $\left(100 \mathrm{MHz}, \mathrm{CDCl}_{3}, 25^{\circ} \mathrm{C}\right): \delta=172.8,62.6,60.6,56.9,38.9$, 33.9, 32.3, 32.0, 23.9, 14.2; HRMS (ESI): calculated for $\mathrm{C}_{10} \mathrm{H}_{19} \mathrm{BrNaO}_{3}{ }^{+}[\mathrm{M}+\mathrm{Na}]^{+}$289.0410, found 289.0417 .<smiles>CCOC(=O)CC(Br)CO</smiles>
4g: $65 \%$ yield; the reaction was performed following the general procedure using the photocatalyst $\mathbf{1 b}(1 \% \mathrm{~mol}, 1.2 \mathrm{mg})$, sodium ascorbate $(0.07 \mathrm{mmol}, 14 \mathrm{mg}), \mathrm{LiBr}(0.4$ $\mathrm{mmol}, 35 \mathrm{mg})$, DMF $(200 \mu \mathrm{L}), 2 \mathrm{~g}(0.4 \mathrm{mmol}, 55 \mu \mathrm{L})$, 3a $(0.2 \mathrm{mmol}, 24 \mu \mathrm{L})$ and $\mathrm{H}_{2} \mathrm{O}(200$ $\mu \mathrm{L}$ ). Purification by flash column chromatography (gradient eluent from 8:2 to 6:4 cyclohexane:AcOEt) afforded the title compound $\mathbf{4 g}$ ( $65 \%$ yield, $37 \mathrm{mg}$ ) as a colourless oil. Spectral properties were according to the literature. ${ }^{5} \mathrm{HRMS}(\mathrm{ESI})$ : calculated for $\mathrm{C}_{11} \mathrm{H}_{21} \mathrm{BrNaO}_{3}{ }^{+}[\mathrm{M}+\mathrm{Na}]^{+} 303.0566$, found 303.0556 . 
$\int_{N C}^{\mathrm{Br}} \int_{4}^{\mathrm{OH}}$

4h: $94 \%$ yield; the reaction was performed following the general procedure using the photocatalyst 1b (1\% mol, $1.2 \mathrm{mg})$, sodium ascorbate $(0.07 \mathrm{mmol}, 14 \mathrm{mg}), \mathrm{LiBr}(0.4 \mathrm{mmol}$, $35 \mathrm{mg}), \mathrm{DMF}(200 \mu \mathrm{L}), 2 \mathrm{~h}(0.4 \mathrm{mmol}, 28 \mu \mathrm{L}), 3 \mathrm{a}(0.2 \mathrm{mmol}, 24 \mu \mathrm{L})$ and $\mathrm{H}_{2} \mathrm{O}(200 \mu \mathrm{L})$. Purification by flash column chromatography (7:3 cyclohexane:AcOEt) afforded the title compound $4 \mathrm{~h}$ (94 $\%$ yield, $41 \mathrm{mg}$ ) as a colourless oil. Spectral properties were according to the literature. ${ }^{5}$ HRMS (ESI): calculated for $\mathrm{C}_{8} \mathrm{H}_{15} \mathrm{BrNO}^{+}[\mathrm{M}+\mathrm{H}]^{+} 220.0332$, found 220.0322 .

$\overbrace{\mathrm{CCl}_{3}}^{\mathrm{Cl}} \mathrm{rr}_{4}^{\mathrm{OH}}$

4i: $68 \%$ yield; the reaction was performed following the general procedure using the photocatalyst $\mathbf{1 b}$ ( $1 \% \mathrm{~mol}, 1.2 \mathrm{mg})$, sodium ascorbate $(0.07 \mathrm{mmol}, 14 \mathrm{mg}), \mathrm{LiCl}(0.4 \mathrm{mmol}, 17$ $\mathrm{mg})$, DMF $(200 \mu \mathrm{L}), \mathbf{2 k}(0.4 \mathrm{mmol}, 40 \mu \mathrm{L}), 3 \mathrm{a}(0.2 \mathrm{mmol}, 24 \mu \mathrm{L})$ and $\mathrm{H}_{2} \mathrm{O}(200 \mu \mathrm{L})$. Purification by flash column chromatography (7:3 cyclohexane:AcOEt) afforded the title compound $\mathbf{4 k}$ (68\% yield, $34 \mathrm{mg}$ ) as a colourless oil. Spectral properties were according to the literature. ${ }^{5}$ Elemental Analysis: Found C, 33.8; $\mathrm{H}, 4.9 \%$. Calc. for $\mathrm{C}_{7} \mathrm{H}_{12} \mathrm{Cl}_{4} \mathrm{O} ; \mathrm{C}, 33.1 ; \mathrm{H}, 4.8 \%$.<smiles>OCC(Br)CC(Br)CO</smiles>
4j: $63 \%$ yield; the reaction was performed following the general procedure using the photocatalyst 1b (1\% mol, $1.2 \mathrm{mg})$, sodium ascorbate $(0.07 \mathrm{mmol}, 14 \mathrm{mg}), \mathrm{LiBr}(0.4 \mathrm{mmol}$, $35 \mathrm{mg})$, DMF (200 $\mu \mathrm{L}), 2 \mathrm{j}(0.4 \mathrm{mmol}, 113 \mathrm{mg})$, 3a $(0.2 \mathrm{mmol}, 24 \mu \mathrm{L})$ and $\mathrm{H}_{2} \mathrm{O}(200 \mu \mathrm{L})$. Purification by flash column chromatography (6:4 cyclohexane:AcOEt) afforded the title compound $\mathbf{4 j}$ (63\% yield, $48 \mathrm{mg}$ ) as a colourless oil. Spectral properties were according to the literature. ${ }^{5}$ HRMS (ESI): calculated for $\mathrm{C}_{8} \mathrm{H}_{15} \mathrm{Br}_{3} \mathrm{NaO}_{2}{ }^{+}[\mathrm{M}+\mathrm{Na}]^{+}$402.8514, found 402.8519 .

$\mathrm{CF}_{3}\left(\mathrm{CF}_{2}\right)_{4} \mathrm{CF}_{2} \mathrm{H}_{4}{ }_{4} \mathrm{OH}$

4k: $35 \%$ yield; the reaction was performed following the general procedure using the photocatalyst $1 \mathbf{b}(1 \% \mathrm{~mol}, 1.2 \mathrm{mg})$, sodium ascorbate $(0.07 \mathrm{mmol}, 14 \mathrm{mg}), \mathrm{LiBr}$ (0.4 mmol, $35 \mathrm{mg})$, DMF (200 $\mu \mathrm{L}), 2 \mathrm{k}(0.4 \mathrm{mmol}, 40 \mu \mathrm{L}), 3 \mathrm{a}(0.2 \mathrm{mmol}, 24 \mu \mathrm{L})$ and $\mathrm{H}_{2} \mathrm{O}(200 \mu \mathrm{L})$. Purification by flash column chromatography (gradient eluent from 8:2 to 6:4 cyclohexane:AcOEt) afforded the title compound $\mathbf{4 k}$ ( $35 \%$ yield, $38 \mathrm{mg}$ ) as a colourless oil. Spectral properties were according to the literature. ${ }^{5}$ Elemental Analysis: Found C, 26.2; $\mathrm{H}, 2.1 \%$; Calc. for $\mathrm{C}_{12} \mathrm{H}_{12} \mathrm{~F}_{13} \mathrm{IO} ; \mathrm{C}, 26.4 ; \mathrm{H}, 2.2 \%$. 


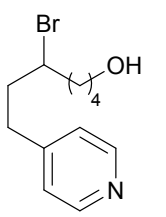

4I: $56 \%$ yield; the reaction was performed following the general procedure using the photocatalyst $\mathbf{1 b}(1 \% \mathrm{~mol}, 1.2 \mathrm{mg})$, sodium ascorbate $(0.07 \mathrm{mmol}, 14 \mathrm{mg})$, LiBr $(0.4 \mathrm{mmol}, 35$ $\mathrm{mg})$, DMF (400 $\mu \mathrm{L}), 2 \mathrm{l}(0.4 \mathrm{mmol}, 101 \mathrm{mg})$, 3a $(0.2 \mathrm{mmol}, 24 \mu \mathrm{L})$ and $\mathrm{H}_{2} \mathrm{O}(400 \mu \mathrm{L})$. After stirring for $20 \mathrm{~h}$, the crude mixture was diluted with $\mathrm{DCM}(15 \mathrm{~mL})$ and $\mathrm{NaOH} 0.1 \mathrm{~N}(15 \mathrm{~mL})$ were added. The phases were separated and the organic layer was extracted with DCM $(2 \times 10 \mathrm{~mL})$. The combined organic layers were washed with brine $(10 \mathrm{~mL})$, dried over $\mathrm{Na}_{2} \mathrm{SO}_{4}$ and concentrated under reduced pressure to give the crude product. The final mixture was purified by flash column chromatography (8:2 AcOEt:cyclohexane) to afford the title compound $4 \mathrm{l}$ ( $56 \%$ yield, $30 \mathrm{mg}$ ) as a colourless oil. ${ }^{1} \mathrm{H}$ NMR (400 $\left.\mathrm{MHz}, \mathrm{CDCl}_{3}, 25^{\circ} \mathrm{C}\right): \delta=8.49$ (bs, $\left.2 \mathrm{H}\right), 7.14(\mathrm{~d}, J=5.2,2 \mathrm{H}), 4.01-3.86(\mathrm{~m}, 1 \mathrm{H}), 3.65(\mathrm{t}, J=5.6,2 \mathrm{H}), 2.96-2.85$ $(\mathrm{m}, 1 \mathrm{H}), 2.74-2.69(\mathrm{~m}, 1 \mathrm{H}), 2.21-1.97(\mathrm{~m}, 2 \mathrm{H}), 1.97-1.78(\mathrm{~m}, 2 \mathrm{H}), 1.73-1.44(\mathrm{~m}, 4 \mathrm{H}) ;{ }^{13} \mathrm{C}$ NMR $(100 \mathrm{MHz}$, $\left.\mathrm{CDCl}_{3}, 25^{\circ} \mathrm{C}\right): \delta 150.0,149.6(2 \mathrm{C}), 124.0(2 \mathrm{C}), 62.3,56.7,39.4,38.9,33.1,32.0,23.9 ; \mathrm{HRMS}$ (ESI): calculated for $\mathrm{C}_{12} \mathrm{H}_{19} \mathrm{BrNO}^{+}[\mathrm{M}+\mathrm{H}]^{+}$272.0645, found 272.0636 .

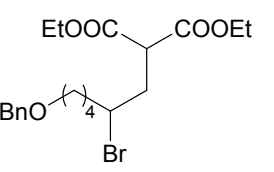

5b: $77 \%$ yield; the reaction was performed following the general procedure using the photocatalyst 1b (1\% mol, $1.2 \mathrm{mg})$, sodium ascorbate $(0.07 \mathrm{mmol}, 14 \mathrm{mg}), \mathrm{LiBr}(0.4$ mmol, $35 \mathrm{mg})$, DMF (200 $\mu \mathrm{L}), 2 \mathrm{a}(0.4 \mathrm{mmol}, 68 \mu \mathrm{L}), 3 \mathbf{b}(0.2 \mathrm{mmol}, 41 \mu \mathrm{L})$ and $\mathrm{H}_{2} \mathrm{O}(200$ $\mu \mathrm{L})$. Purification by flash column chromatography (95:5 cyclohexane:AcOEt) afforded the title compound $\mathbf{5 b}$ (77\% yield, $66 \mathrm{mg}$ ) as a colourless oil. Spectral properties were according to the literature. ${ }^{6}$ HRMS (ESI): calculated for $\mathrm{C}_{20} \mathrm{H}_{29} \mathrm{BrNaO}_{5}{ }^{+}[\mathrm{M}+\mathrm{Na}]^{+}$451.1091, found 451.1083.

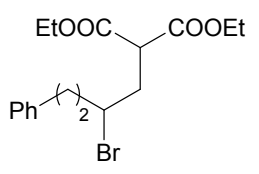

5c: $62 \%$ yield; the reaction was performed following the general procedure using the photocatalyst $1 \mathrm{~b}$ ( $1 \% \mathrm{~mol}, 1.2 \mathrm{mg})$, sodium ascorbate $(0.07 \mathrm{mmol}, 14 \mathrm{mg}), \mathrm{LiBr}(0.4 \mathrm{mmol}$, $35 \mathrm{mg}), \mathrm{CH}_{3} \mathrm{CN}(200 \mu \mathrm{L}), 2 \mathrm{a}(0.4 \mathrm{mmol}, 68 \mu \mathrm{L}), 3 \mathrm{c}(0.2 \mathrm{mmol}, 30 \mu \mathrm{L})$ and $\mathrm{MeOH}(200 \mu \mathrm{L})$. Purification by flash column chromatography (95:5 cyclohexane:AcOEt) afforded the title compound 5c (62\% yield, $46 \mathrm{mg}$ ) as a colourless oil. Spectral properties were according to the literature. ${ }^{5}$ HRMS (ESI): calculated for $\mathrm{C}_{17} \mathrm{H}_{23} \mathrm{BrNaO}_{4}{ }^{+}[\mathrm{M}+\mathrm{Na}]^{+}$393.0672, found 393.0679.

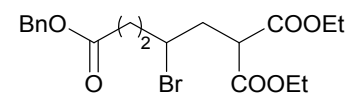

5d: $46 \%$ yield; the reaction was performed following the general procedure using the photocatalyst $1 \mathrm{~b}(1 \% \mathrm{~mol}, 1.2 \mathrm{mg})$, sodium ascorbate $(0.07 \mathrm{mmol}, 14 \mathrm{mg}), \mathrm{LiBr}$ (0.4 mmol, $35 \mathrm{mg})$, DMF (200 $\mu \mathrm{L}), 2 \mathrm{a}(0.4 \mathrm{mmol}, 68 \mu \mathrm{L}), 3 \mathrm{~d}(0.2 \mathrm{mmol}, 41 \mu \mathrm{L})$ and $\mathrm{H}_{2} \mathrm{O}(200 \mu \mathrm{L})$. Purification by flash column chromatography ( $95: 5$ cyclohexane:AcOEt) afforded the title compound $\mathbf{5 d}$ ( $46 \%$ yield, 40 
$\mathrm{mg})$ as a colourless oil; ${ }^{1} \mathrm{H}$ NMR $\left(400 \mathrm{MHz}, \mathrm{CDCl}_{3}, 25^{\circ} \mathrm{C}\right): \delta=7.40-7.32(\mathrm{~m}, 5 \mathrm{H}), 5.10(\mathrm{~s}, 2 \mathrm{H}), 4.28-4.14(\mathrm{~m}$, 4H), $4.13-3.99(\mathrm{~m}, 1 \mathrm{H}), 3.77(\mathrm{dd}, J=10.0,4.4,1 \mathrm{H}), 2.75-2.62(\mathrm{~m}, 1 \mathrm{H}), 2.62-2.54(\mathrm{~m}, 1 \mathrm{H}), 2.54-2.42(\mathrm{~m}$, 1H), $2.37-2.20(\mathrm{~m}, 2 \mathrm{H}), 2.18-2.06(\mathrm{~m}, 1 \mathrm{H}), 1.31-1.25(\mathrm{~m}, 6 \mathrm{H}) ;{ }^{13} \mathrm{C}$ NMR $\left(100 \mathrm{MHz}, \mathrm{CDCl}_{3}, 25{ }^{\circ} \mathrm{C}\right): 172.2$, $168.8,168.5,135.7,128.5$ (2C), 128.3, 128.2 (2C), 66.4, 61.7, 61.7, 53.4, 50.4, 37.8, 34.1, 32.2, 14.0, 14.0; HRMS (ESI): calculated for $\mathrm{C}_{19} \mathrm{H}_{25} \mathrm{BrNaO}_{6}{ }^{+}[\mathrm{M}+\mathrm{Na}]^{+} 451.0727$, found 451.0720 .

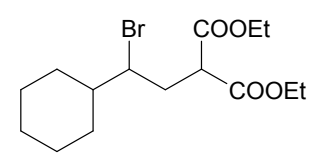

5e: $64 \%$ yield; the reaction was performed following the general procedure using the photocatalyst 1b (1\% mol, $1.2 \mathrm{mg})$, sodium ascorbate $(0.07 \mathrm{mmol}, 14 \mathrm{mg}), \mathrm{LiBr}(0.4$ $\mathrm{mmol}, 35 \mathrm{mg}), \mathrm{CH}_{3} \mathrm{CN}(200 \mu \mathrm{L}), 2 \mathrm{a}(0.4 \mathrm{mmol}, 68 \mu \mathrm{L}), 3 \mathrm{e}(0.2 \mathrm{mmol}, 27 \mu \mathrm{L})$ and $\mathrm{MeOH}$ $(200 \mu \mathrm{L})$. Purification by flash column chromatography (20:1 cyclohexane:AcOEt) afforded the title compound 5 e (64\% yield, $45 \mathrm{mg}$ ) as a colourless oil. Spectral properties were according to the literature. ${ }^{5}$ HRMS (ESI): calculated for $\mathrm{C}_{15} \mathrm{H}_{25} \mathrm{BrNaO}_{4}{ }^{+}[\mathrm{M}+\mathrm{Na}]^{+} 371.0828$, found 371.0832 .

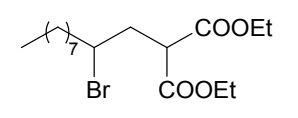

5f: $49 \%$ yield; the reaction was performed following the general procedure using the photocatalyst $1 \mathrm{~b}$ ( $1 \% \mathrm{~mol}, 1.2 \mathrm{mg})$, sodium ascorbate $(0.07 \mathrm{mmol}, 14 \mathrm{mg}), \mathrm{LiBr}(0.4$ $\mathrm{mmol}, 35 \mathrm{mg}), \mathrm{CH}_{3} \mathrm{CN}(200 \mu \mathrm{L}), 2 \mathrm{a}(0.4 \mathrm{mmol}, 68 \mu \mathrm{L}), 3 \mathrm{f}(0.2 \mathrm{mmol}, 38 \mu \mathrm{L})$ and $\mathrm{MeOH}(200 \mu \mathrm{L})$. Purification by flash column chromatography (20:1 cyclohexane:AcOEt) afforded the title compound $\mathbf{5 f}$ ( $49 \%$ yield, 37 $\mathrm{mg}$ ) as a colourless oil. Spectral properties were according to the literature. ${ }^{5}$ HRMS (ESI): calculated for $\mathrm{C}_{17} \mathrm{H}_{31} \mathrm{BrNaO}_{4}{ }^{+}[\mathrm{M}+\mathrm{Na}]^{+} 401.1298$, found 401.1308.

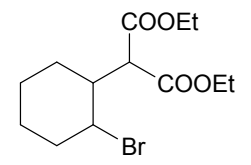

5g: $68 \%$ yield; the reaction was performed following the general procedure using the photocatalyst $1 \mathbf{b}$ ( $1 \% \mathrm{~mol}, 1.2 \mathrm{mg})$, sodium ascorbate $(0.07 \mathrm{mmol}, 14 \mathrm{mg}), \mathrm{LiBr}(0.4 \mathrm{mmol}$, $35 \mathrm{mg}), \mathrm{DMF}(200 \mu \mathrm{L}), 2 \mathrm{a}(0.4 \mathrm{mmol}, 68 \mu \mathrm{L}), 3 \mathrm{~g}(0.2 \mathrm{mmol}, 20 \mu \mathrm{L})$ and $\mathrm{H}_{2} \mathrm{O}(200 \mu \mathrm{L})$. Purification by flash column chromatography (20:1 cyclohexane:AcOEt) afforded the title compound $\mathbf{5 g}$ (64\% yield, $43 \mathrm{mg}$ ) as a colourless oil. Spectral properties were according to literature..$^{5} \mathrm{HRMS}$ (ESI): calculated for $\mathrm{C}_{13} \mathrm{H}_{21} \mathrm{BrNaO}_{4}{ }^{+}[\mathrm{M}+\mathrm{Na}]^{+}$343.0515, found 343.0522 .

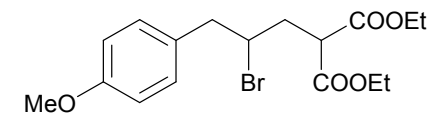

5h: $58 \%$ yield; the reaction was performed following the general procedure using the photocatalyst $\mathbf{1 b}(1 \% \mathrm{~mol}, 1.2 \mathrm{mg})$, sodium ascorbate $(0.07 \mathrm{mmol}, 14$ $\mathrm{mg})$, LiBr (0.4 mmol, $35 \mathrm{mg})$, DMF (200 $\mu \mathrm{L}), 2 \mathrm{a}(0.4 \mathrm{mmol}, 68 \mu \mathrm{L})$, 3h $(0.2 \mathrm{mmol}, 31 \mu \mathrm{L})$ and $\mathrm{H}_{2} \mathrm{O}(200 \mu \mathrm{L})$. 
Purification by flash column chromatography (95:5 cyclohexane:acetone) afforded the title compound $\mathbf{5 h}$ ( $58 \%$ yield, $44 \mathrm{mg}$ ) as a colourless oil; ${ }^{1} \mathrm{H}$ NMR $\left(400 \mathrm{MHz}, \mathrm{CDCl}_{3}, 25^{\circ} \mathrm{C}\right): \delta=7.17-7.10(\mathrm{~m}, 2 \mathrm{H}), 6.89-6.81$ $(\mathrm{m}, 2 \mathrm{H}), 4.27-4.08(\mathrm{~m}, 5 \mathrm{H}), 3.81-3.74(\mathrm{~m}, 1 \mathrm{H}), 3.77(\mathrm{~s}, 3 \mathrm{H}), 3.24-3.07(\mathrm{~m}, 2 \mathrm{H}), 2.50(\mathrm{tdd}, \mathrm{J}=19.4,13.5,5.8$, 1H), 2.23 (ddd, J=14.9, 11.0, 4.0, 1H), $1.32-1.18(\mathrm{~m}, 6 \mathrm{H}) ;{ }^{13} \mathrm{C} \mathrm{NMR}\left(100 \mathrm{MHz}, \mathrm{CDCl}_{3}, 25^{\circ} \mathrm{C}\right): \delta=168.9,168.8$, 158.5, 130.2 (2C), 129.8, 113.8 (2C), 61.7, 61.6, 55.2, 54.6, 50.6, 44.9, 37.0, 14.0 (2C); HRMS (ESI): calculated for $\mathrm{C}_{17} \mathrm{H}_{23} \mathrm{BrNaO}_{5}{ }^{+}[\mathrm{M}+\mathrm{Na}]^{+} 409.0621$, found 409.0616 .

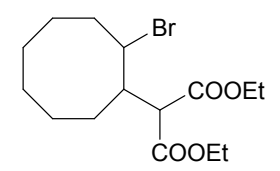

5i: $45 \%$ yield; the reaction was performed following the general procedure using the photocatalyst $\mathbf{1 b}(1 \% \mathrm{~mol}, 1.2 \mathrm{mg})$, sodium ascorbate $(0.07 \mathrm{mmol}, 14 \mathrm{mg}), \mathrm{LiBr}(0.4$ mmol, $35 \mathrm{mg}), \mathrm{CH}_{3} \mathrm{CN}(200 \mu \mathrm{L}), 2 \mathrm{a}(0.4 \mathrm{mmol}, 68 \mu \mathrm{L}), 3 \mathbf{i}(0.2 \mathrm{mmol}, 26 \mu \mathrm{L})$ and $\mathrm{MeOH}$ $(200 \mu \mathrm{L})$. Purification by flash column chromatography (30:1 cyclohexane:AcOEt) afforded the title compound $\mathbf{5 i}$ (45\% yield, $31 \mathrm{mg}$ ) as a colourless oil. Spectral properties were according to the literature. ${ }^{5}$ HRMS (ESI): calculated for $\mathrm{C}_{15} \mathrm{H}_{25} \mathrm{BrNaO}_{4}{ }^{+}[\mathrm{M}+\mathrm{Na}]^{+} 371.0828$, found 371.0834 .

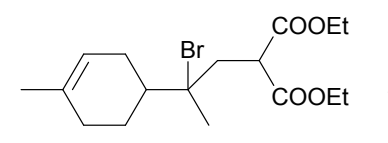

5j: $47 \%$ yield; the reaction was performed following the general procedure using the photocatalyst $\mathbf{1 b}(1 \% \mathrm{~mol}, 1.2 \mathrm{mg})$, sodium ascorbate $(0.07 \mathrm{mmol}, 14 \mathrm{mg}), \mathrm{LiBr}$ (0.4 mmol, $35 \mathrm{mg})$, DMF (200 $\mu \mathrm{L}), 2 \mathrm{a}(0.4 \mathrm{mmol}, 68 \mu \mathrm{L}), 3 \mathrm{j}(0.2 \mathrm{mmol}, 32 \mu \mathrm{L})$ and $\mathrm{H}_{2} \mathrm{O}(200 \mu \mathrm{L})$. Purification by flash column chromatography (9:1 cyclohexane:acetone) afforded the title compound $\mathbf{5 j}$ ( $47 \%$ yield, 35 $\mathrm{mg}$ ) as a colourless oil. Spectral properties were according to the literature. ${ }^{5}$ HRMS (ESI): calculated for $\mathrm{C}_{17} \mathrm{H}_{27} \mathrm{BrNaO}_{4}^{+}[\mathrm{M}+\mathrm{Na}]^{+}$397.0985, found 397.0990.
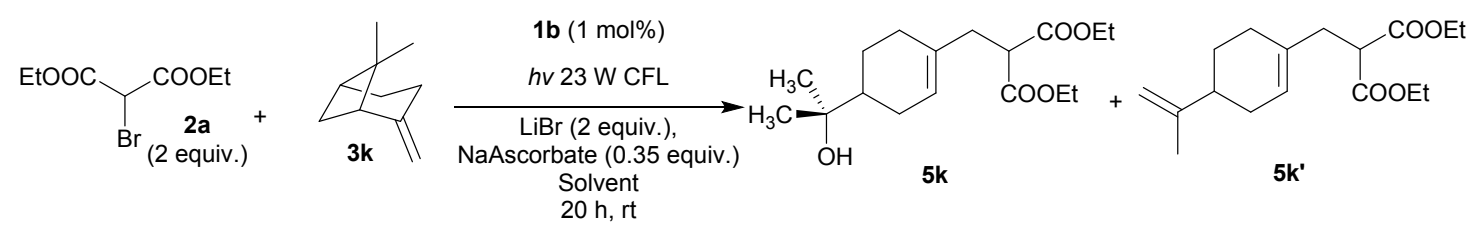

The reaction was performed following the general procedure using the photocatalyst $\mathbf{1 b}$ (1\% mol, $1.2 \mathrm{mg}$ ), sodium ascorbate (0.07 mmol, $14 \mathrm{mg}), \mathrm{LiBr}(0.4 \mathrm{mmol}, 35 \mathrm{mg})$, DMF (200 $\mu \mathrm{L}), 2 \mathrm{a}(0.4 \mathrm{mmol}, 68 \mu \mathrm{L}), 3 \mathbf{k}(1 \mathrm{~S})$ (-)- $\beta$-pinene $\left(0.2 \mathrm{mmol}, 32 \mu \mathrm{L}\right.$ ) and $\mathrm{H}_{2} \mathrm{O}(200 \mu \mathrm{L})$. Purification by flash column chromatography (95:5 to 50:50 cyclohexane:AcOEt) afforded the title compounds $\mathbf{5 k}$ ( $29 \%$ yield) and $\mathbf{5} \mathbf{k}^{\mathbf{\prime}}$ ( $18 \%$ yield) as colourless oils. 
<smiles>CCOC(=O)C(CC1=CCC(C(C)(C)O)CC1)C(=O)OCC</smiles>

(5k): ${ }^{1} \mathrm{H}$ NMR $\left(400 \mathrm{MHz}, \mathrm{CDCl}_{3}, 25^{\circ} \mathrm{C}\right): \delta=5.47-5.36(\mathrm{~m}, 1 \mathrm{H}), 4.15(\mathrm{q}, J=7.1,4 \mathrm{H})$, $3.50(\mathrm{t}, J=7.9,1 \mathrm{H}), 2.52(\mathrm{~d}, J=7.8,2 \mathrm{H}), 2.13-1.92(\mathrm{~m}, 3 \mathrm{H}), 1.91-1.82(\mathrm{~m}, 1 \mathrm{H})$, $1.82-1.68(\mathrm{~m}, 1 \mathrm{H}), 1.51-1.39(\mathrm{~m}, 1 \mathrm{H}), 1.25-1.20(\mathrm{~m}, 7 \mathrm{H}), 1.15(\mathrm{~s}, 3 \mathrm{H}), 1.13(\mathrm{~s}$, $3 \mathrm{H}) ;{ }^{13} \mathrm{C} \mathrm{NMR}\left(100 \mathrm{MHz}, \mathrm{CDCl}_{3}, 25^{\circ} \mathrm{C}\right): \delta=169.3,169.2,133.7,123.2,72.6,61.3(2 \mathrm{C}), 50.7,44.8,36.4,28.9$, 27.4, 26.8, 26.3, 23.8, 14.1 (2C); HRMS (ESI): calculated for $\mathrm{C}_{17} \mathrm{H}_{29} \mathrm{O}_{5}{ }^{+}[\mathrm{M}+\mathrm{H}]^{+}$313.2010, found 313.2004.

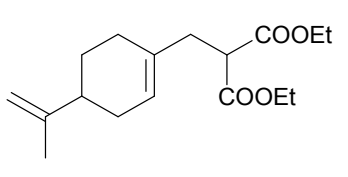

$\left(5 k^{\prime}\right):{ }^{1} \mathrm{H}$ NMR $\left(400 \mathrm{MHz}, \mathrm{CDCl}_{3}, 25^{\circ} \mathrm{C}\right): \delta 5.50-5.46(\mathrm{~m}, 1 \mathrm{H}), 4.74-4.66(\mathrm{~m}, 2 \mathrm{H})$, $4.19(\mathrm{q}, J=7.1,4 \mathrm{H}), 3.54(\mathrm{t}, J=7.9,1 \mathrm{H}), 2.56(\mathrm{~d}, J=7.8,2 \mathrm{H}), 2.18-1.95(\mathrm{~m}, 4 \mathrm{H}), 1.95-$ $1.85(\mathrm{~m}, 1 \mathrm{H}), 1.85-1.76(\mathrm{~m}, 1 \mathrm{H}), 1.72(\mathrm{~s}, 3 \mathrm{H}), 1.44(\mathrm{tdd}, \mathrm{J}=13.3,11.4,5.8,1 \mathrm{H}), 1.29$ - $1.23(\mathrm{~m}, 6 \mathrm{H}) ;{ }^{13} \mathrm{C}$ NMR $\left(100 \mathrm{MHz}, \mathrm{CDCl}_{3}, 25^{\circ} \mathrm{C}\right): \delta=169.3(2 \mathrm{C}), 149.8,133.4,123.2,108.6,61.3(2 \mathrm{C}), 50.7$, $40.9,36.5,30.7,28.4,27.7,20.8,14.1(2 C)$.
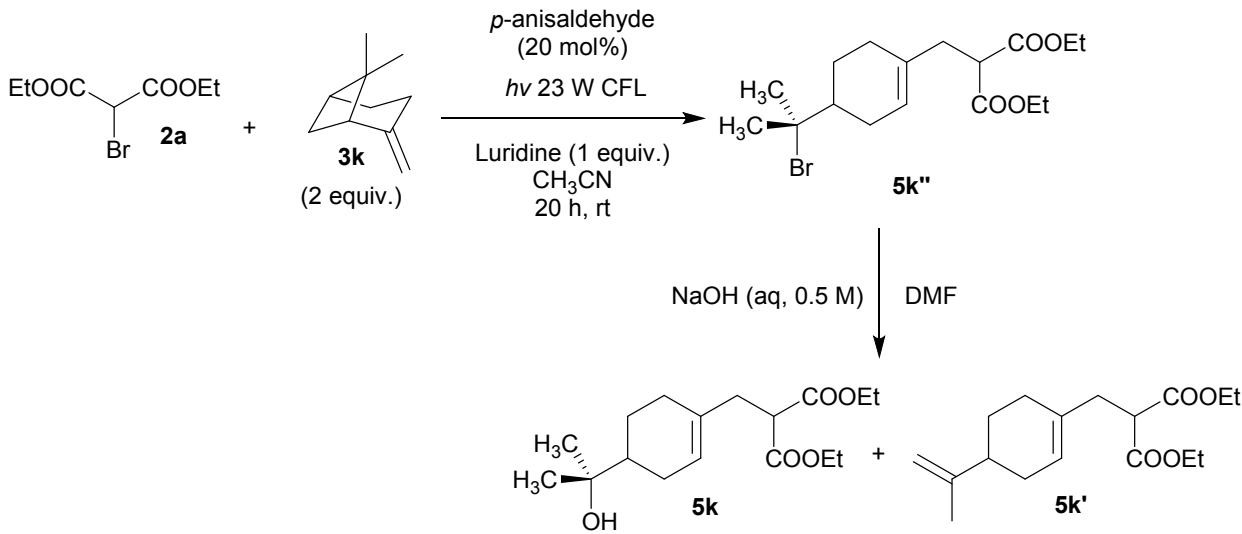

Structures of compounds $\mathbf{5 k}$ and $\mathbf{5} \mathbf{k}^{\prime}$ were confirmed by chemical correlation and NMR spectroscopy $\left({ }^{1} \mathrm{H}\right.$, ${ }^{13}$ C, COSY, HSQC NMR experiments). Compound 5k" was prepared following the procedure reported by Melchiorre $^{5}(0.1 \mathrm{mmol}$ of (-)- $\beta$-pinene). Then the reaction crude was dissolved in DMF $(2 \mathrm{~mL})$ and treated with an aqueous solution of $\mathrm{NaOH}(0.5 \mathrm{M}, 2 \mathrm{~mL})$. After 3 hours the mixture was transferred in a separator funnel and extracted with AcOEt $(3 \times 5 \mathrm{~mL})$. The combined organic layers were washed with brine $(10 \mathrm{~mL})$, dried over $\mathrm{Na}_{2} \mathrm{SO}_{4}$ and concentrated under reduced pressure to give a mixture of $\mathbf{5 k}$ and $\mathbf{5 \mathbf { k } ^ { \prime }}$ in $36: 64$ ratio with complete conversion of $\mathbf{5 k}$ ". 


\section{Evidences of radical mechanism}

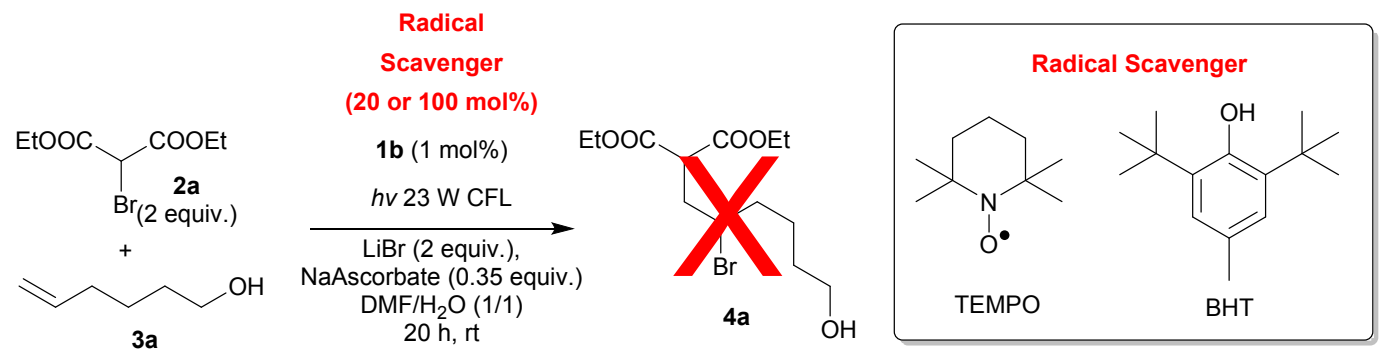

Evidence of a possible radical mechanism was highlighted performing the reaction in presence of radical scavengers. The experiments were performed using general procedure in the presence of catalytic (20 mol\%) and stoichiometric amount of TEMPO (2,2,6,6-tetramethylpiperidine 1-oxyl) or BHT (3,5-di-tertbutylhydroxytoluene). In both cases no formation of the desired product or adducts between TEMPO and reaction intermediates were observed.

\section{Photophysical measurements}

Photochemical experiments were carried out at room temperature in deaerated solutions. All absorption spectra were recorded in a quartz cuvette (optical pathlength $0.1 \mathrm{~cm}$ ) with a UV/VIS spectrophotometer Perkin Elmer Lambda 650. Luminescence spectra were performed with a PerkinElmer LS-55. Lifetimes of 1b with increasing amount of NaAscorbate were measured by Edinburgh FLS920 spectrofluorimeter equipped with a TCC900 card for data acquisition in time-correlated single-photon counting experiments ( $0.5 \mathrm{~ns}$ time resolution) with a PicoQuant pulsed diode laser $340 \pm 20 \mathrm{~nm}$. 


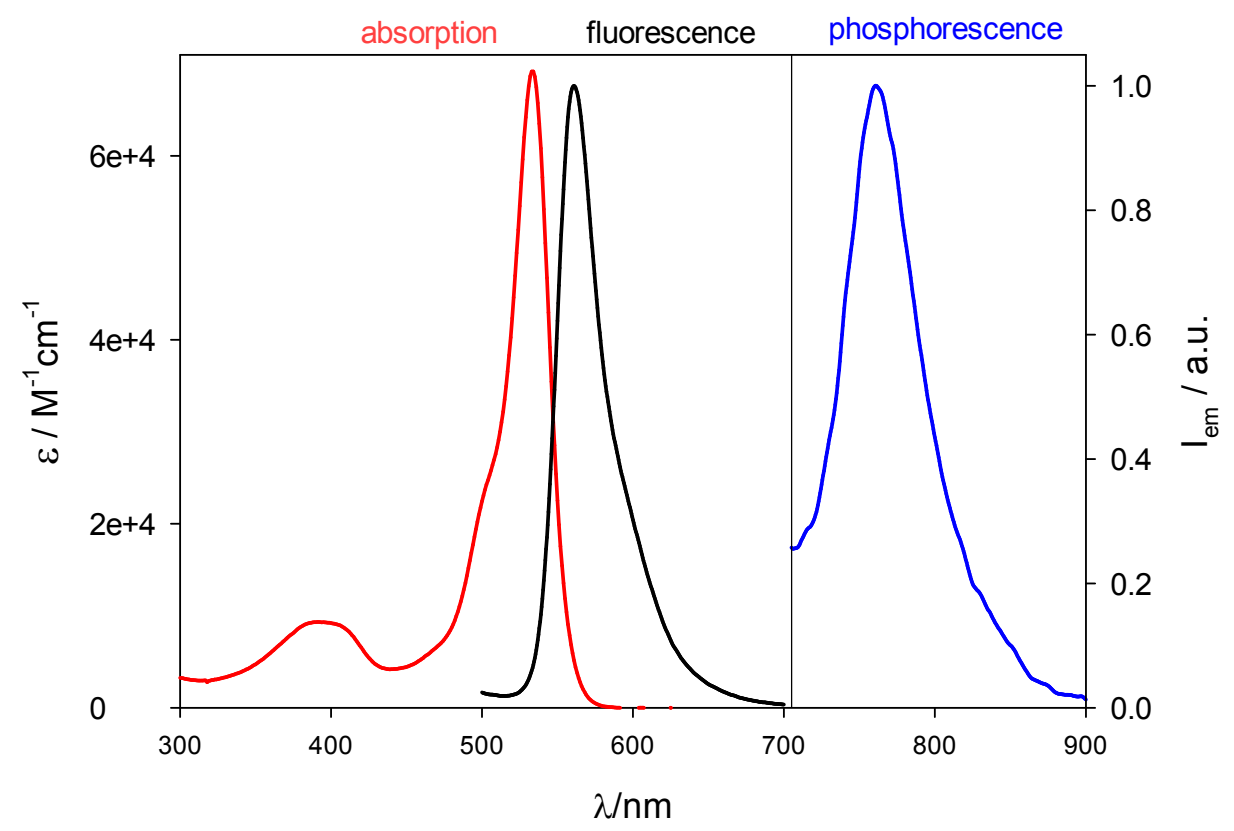

Figure S1. Absorption (red solid line) and fluorescence emission (black solid line, $\lambda_{\text {ex }} 405 \mathrm{~nm}$ ) spectra of $\mathbf{1 b}$ in DMF Uvasol ${ }^{\circledR}$ at $298 \mathrm{~K}$. The phosphorescence emission spectrum (blue solid lines) was obtained after 5 freeze-pump cycles $\left(\lambda_{\mathrm{ex}} 525 \mathrm{~nm}\right)$.

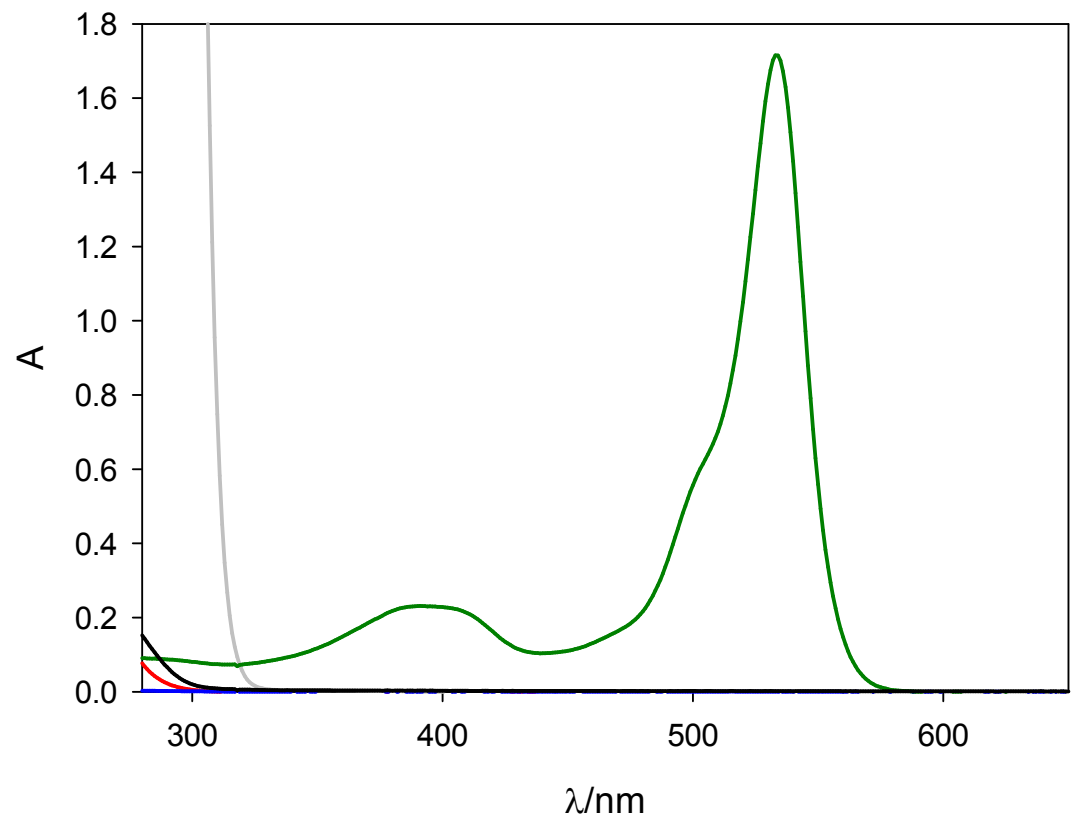

Figure S2. Absorption spectra of 5-hexenol $0.25 \mathrm{M}$ (blue solid line), diethyl 2-bromo-malonate $0.5 \mathrm{M}$ (red solid line), sodium ascorbate $0.088 \mathrm{M}$ (grey solid line), lithium bromide $0.5 \mathrm{M}$ (black solid line) and $\mathbf{1 b} 2.5 \times$ 
$10^{-4} \mathrm{M}$ (dark green solid line) in DMF Uvaso ${ }^{\circledR}: \mathrm{H}_{2} \mathrm{O}$ ratio $1: 1$. The amount of the species in solution is the same used in reaction mixture condition, a part from $\mathbf{1 b}$, that is ten times more diluted.

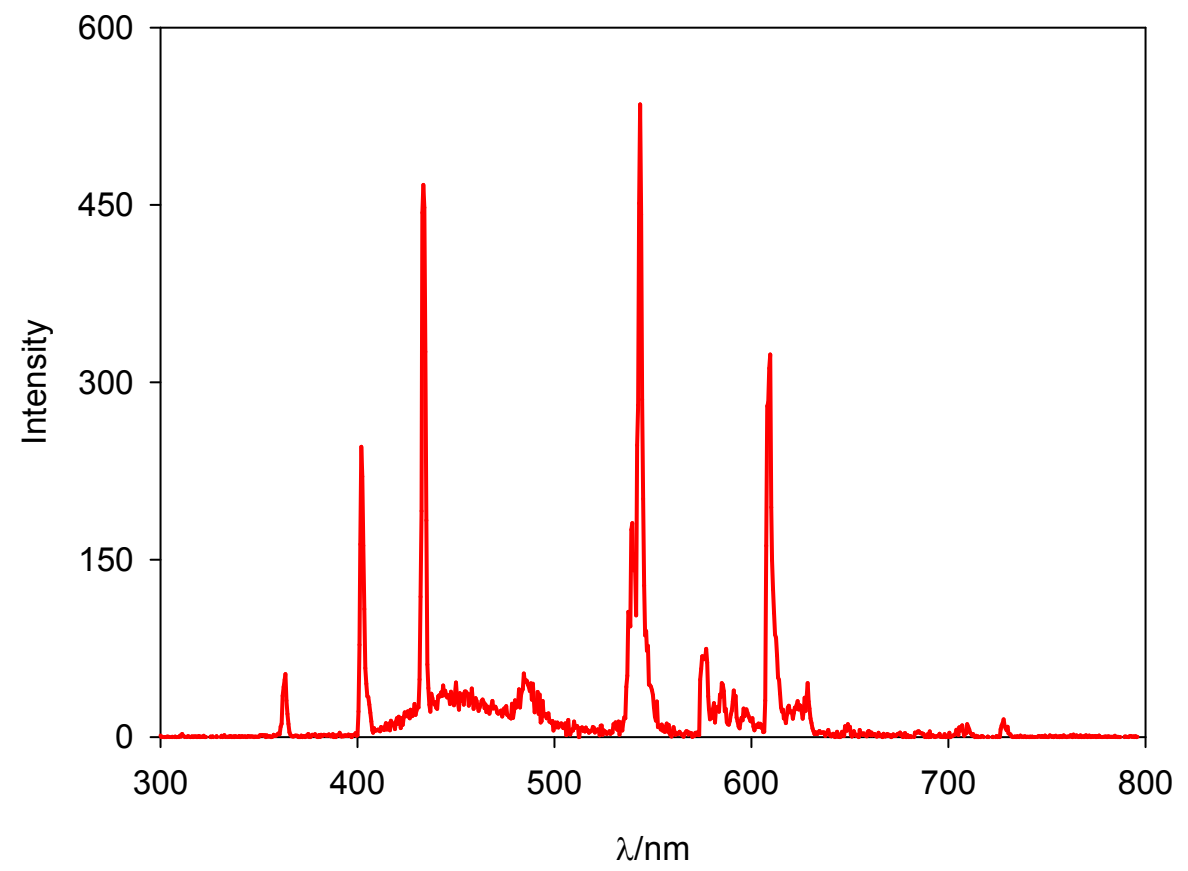

Figure S3. Emission profile of the 23W Compact Fluorescent lamp used to irradiate the solutions.

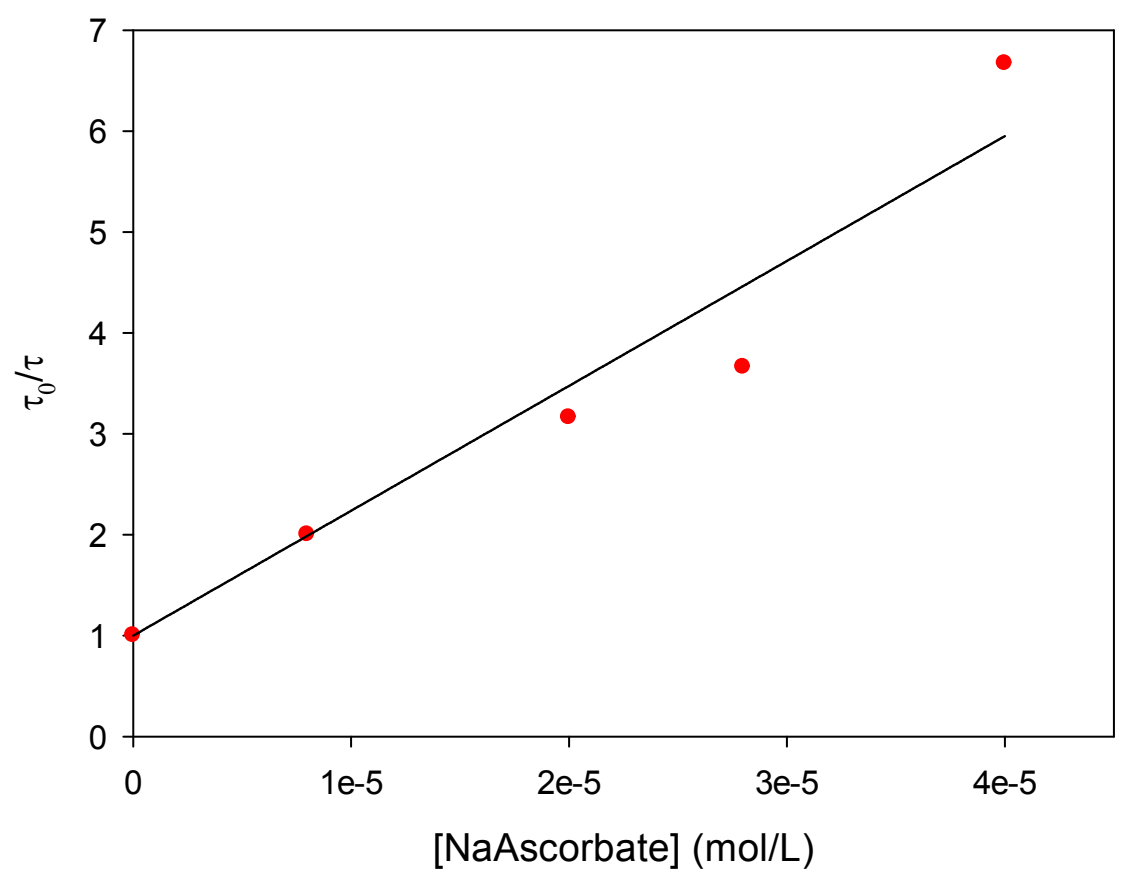

Figure S4. Stern-Volmer quenching plot. After every addition of NaAscorbate, the solution undergoes 5 freeze-pump cycles, before collecting the phosphorescence lifetime. 
The Stern-Volmer plot shows a linear correlation between the amounts of NaAscorbate and the ratio $\tau_{0} / \tau$. On the basis of the Stern-Volmer equation (1), it is possible to calculate the quenching constant:

(1) $\tau_{0} / \tau=1+K_{s V}[Q]=1+k_{q} \tau_{0}[Q]$

We calculated a quenching constant $\mathrm{k}_{\mathrm{q}}$ of $5.5 \times 10^{8} \mathrm{M}^{-1} \mathrm{~s}^{-1}$. The estimated value of $\mathrm{k}_{\mathrm{q}}$ is high and close to the diffusion limit.

Reaction in presence of triethylamine
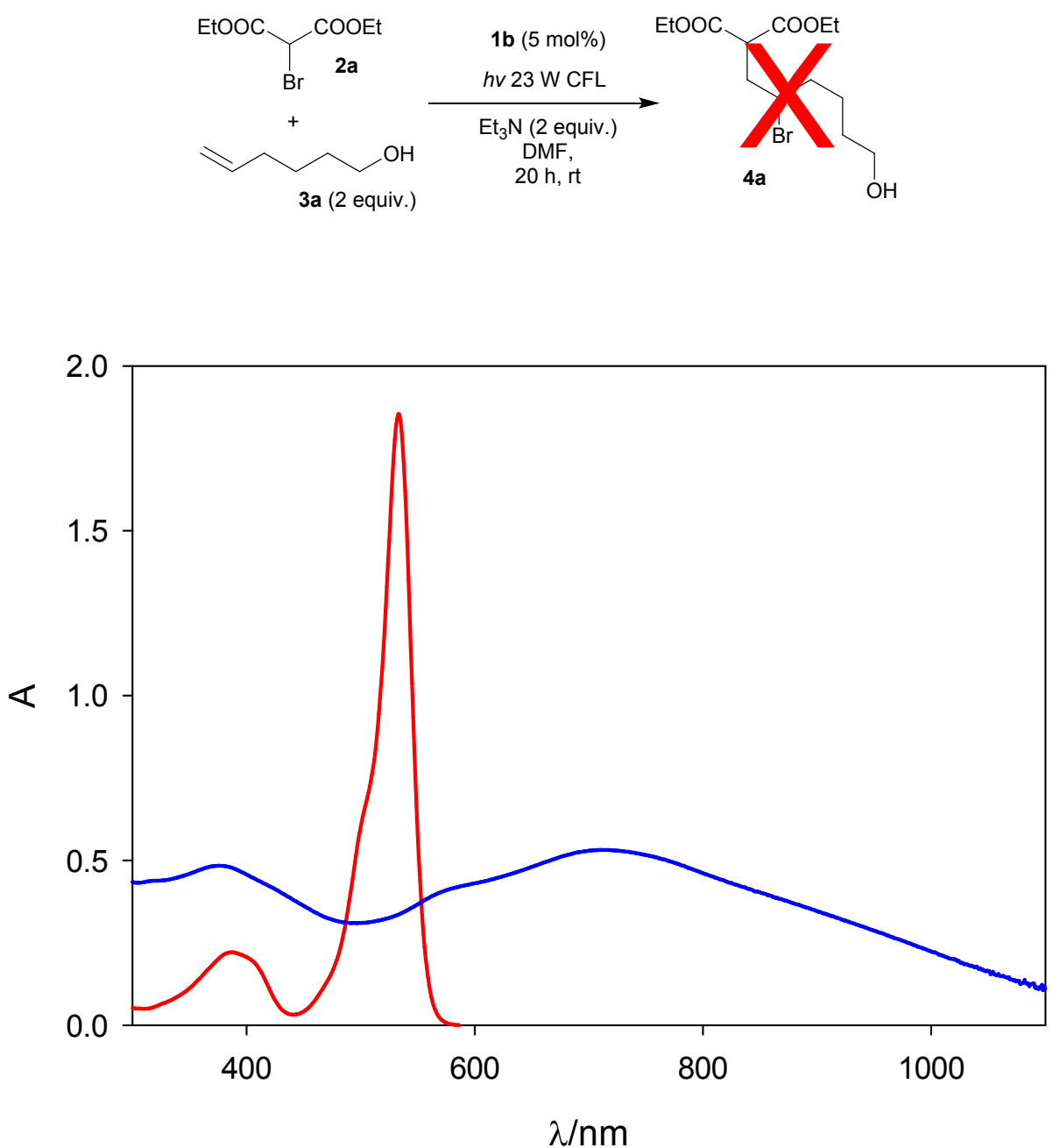

Figure S5. Absorption spectra of reaction mixture containing 3a $0.01 \mathrm{M}$, 2a $0.005 \mathrm{M}$, 1 f $2.5 \times 10^{-4}$ $\mathrm{M}$ (red solid line), and upon addition of $\mathrm{Et}_{3} \mathrm{~N} 0.01 \mathrm{M}$ and irradiation with $23 \mathrm{~W}$ Compact Fluorescent lamp (blue solid line) in DMF Uvasol@. The amount of the species in solution is a hundred times more diluted respect to the reaction mixture. 


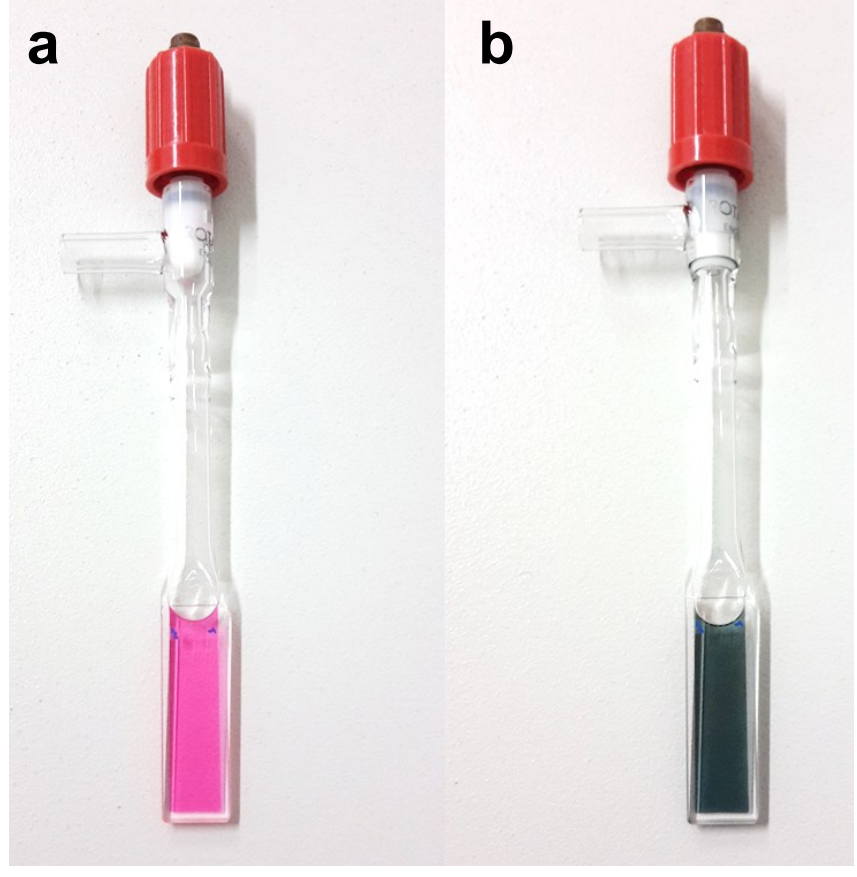

Figure S6. Reaction mixture without $\mathrm{Et}_{3} \mathrm{~N}$ (cuvette on the left) and after addition of the amine and 30 minutes of irradiation with 23W Compact Fluorescent lamp (cuvette on the right).

\section{References:}

1 C. Zhang, J. Zhao, S. Wu, Z. Wang, W. Wu, J. Ma, S. Guo and L. Huang, J. Am. Chem. Soc. 2013, 135, 10566-10578.

2 L. Zoli and P. G. Cozzi, ChemSusChem 2009, 2, 218-220.

3 I. Taisuk, M. Takumi, S. Yohei and K. Motomu Chem._Eur. J. 2015, 21, 15955-15959.

4 A. Palani, J. Su, D. Xiao, X. Huang, A. U. Rao, X. Chen, H. Tang, J. Qin, Y. R. Huang, R. G. Aslanian, B. A. Mckittrick and S. Degrado, U.S. Pat. Appl. Publ. 238 pp., 2008, US 20080019978 A1.

5 E. Arceo, E. Montroni and P. Melchiorre Angew. Chem. Int. Ed. 2014, 53, $12064-12068$.

6 J. D. Nguyen, J.W. Tucker, M. D. Konieczynska and C. R. J. Stephenson, J. Am. Chem. Soc. 2011, 133, 4160-4163. 


\section{Copies of NMR spectra}

$\int_{\text {EtooC }}^{\mathrm{Br}} \int_{4 a}^{\mathrm{E}_{4} \mathrm{OH}}$

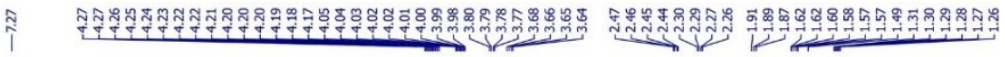

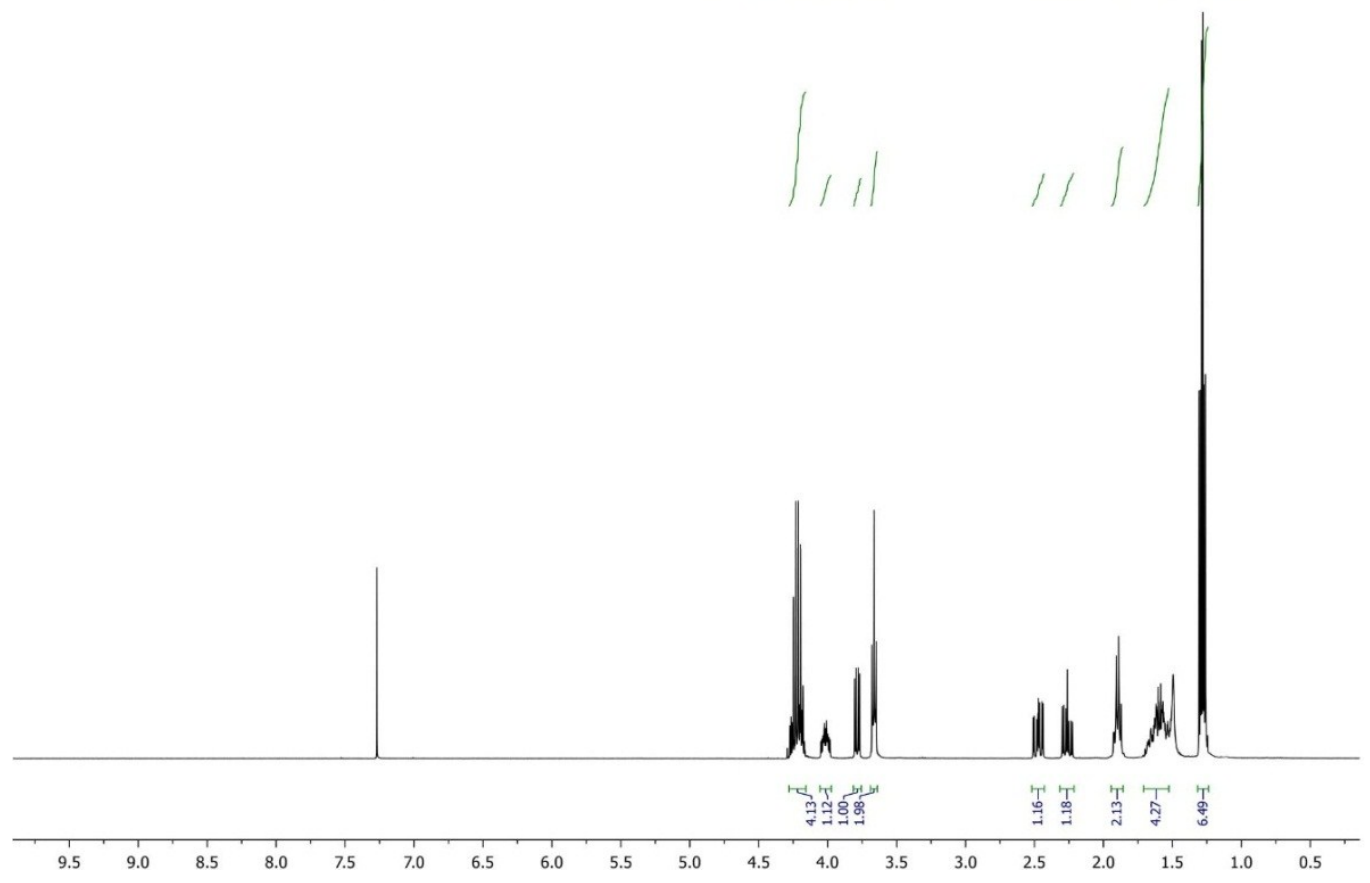

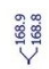

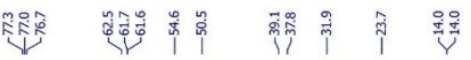

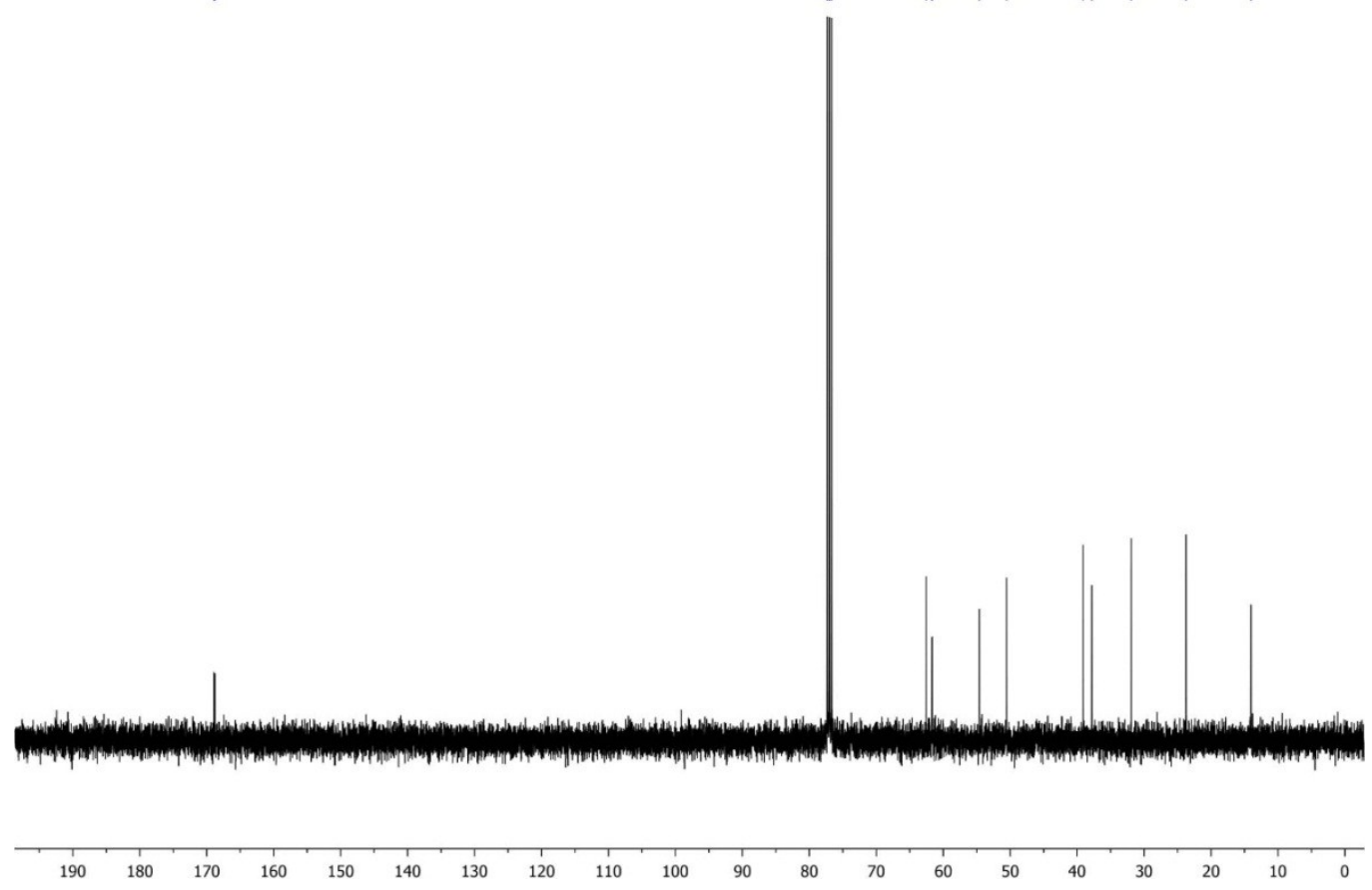


$\overbrace{\mathrm{EtOOCC}}^{\mathrm{Br}} \int_{\mathrm{CH}_{4}}^{\mathrm{OH}}$

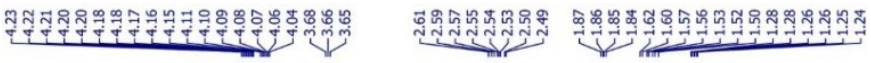
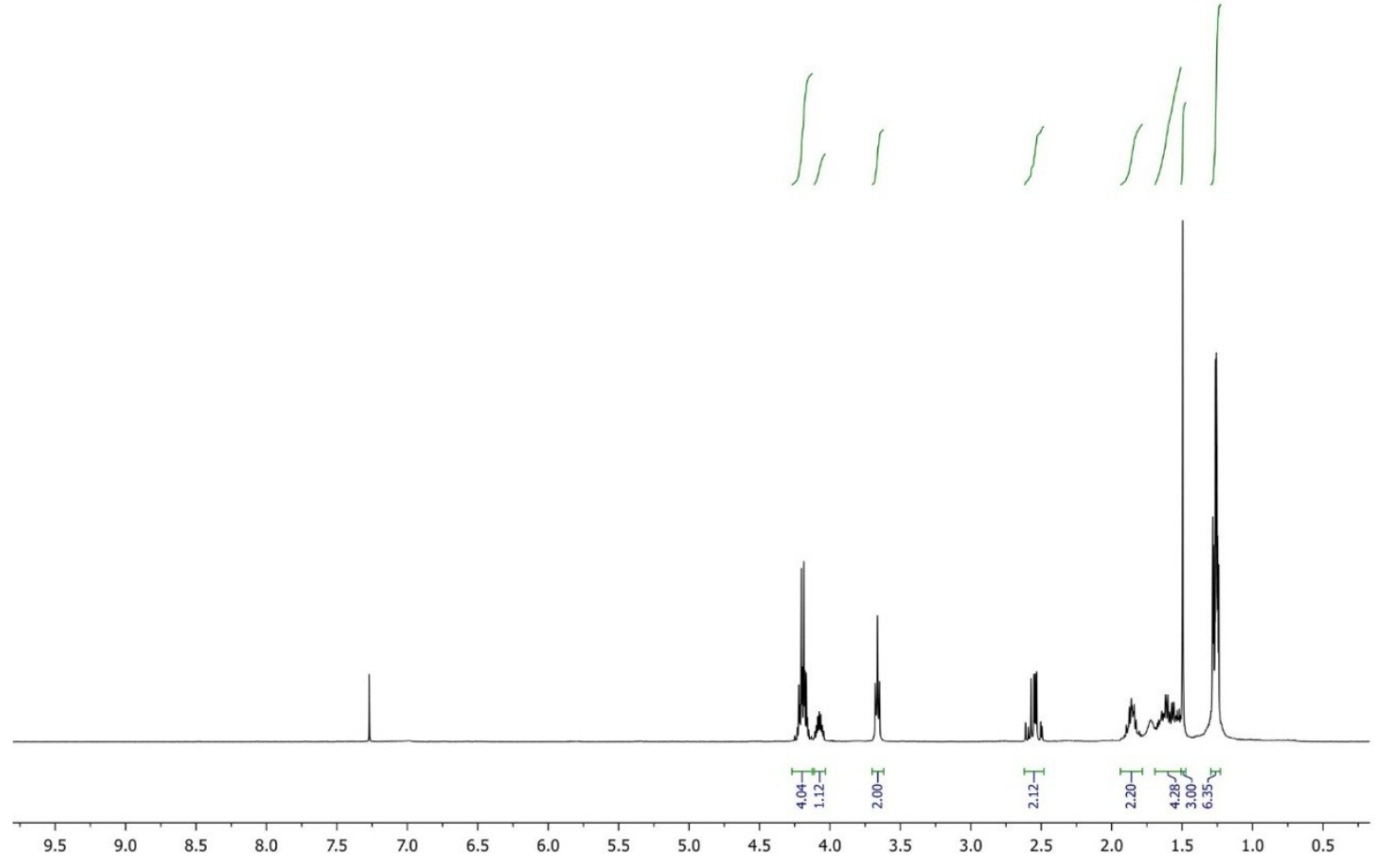

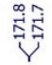

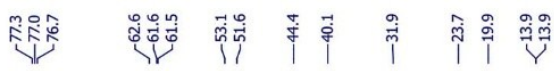
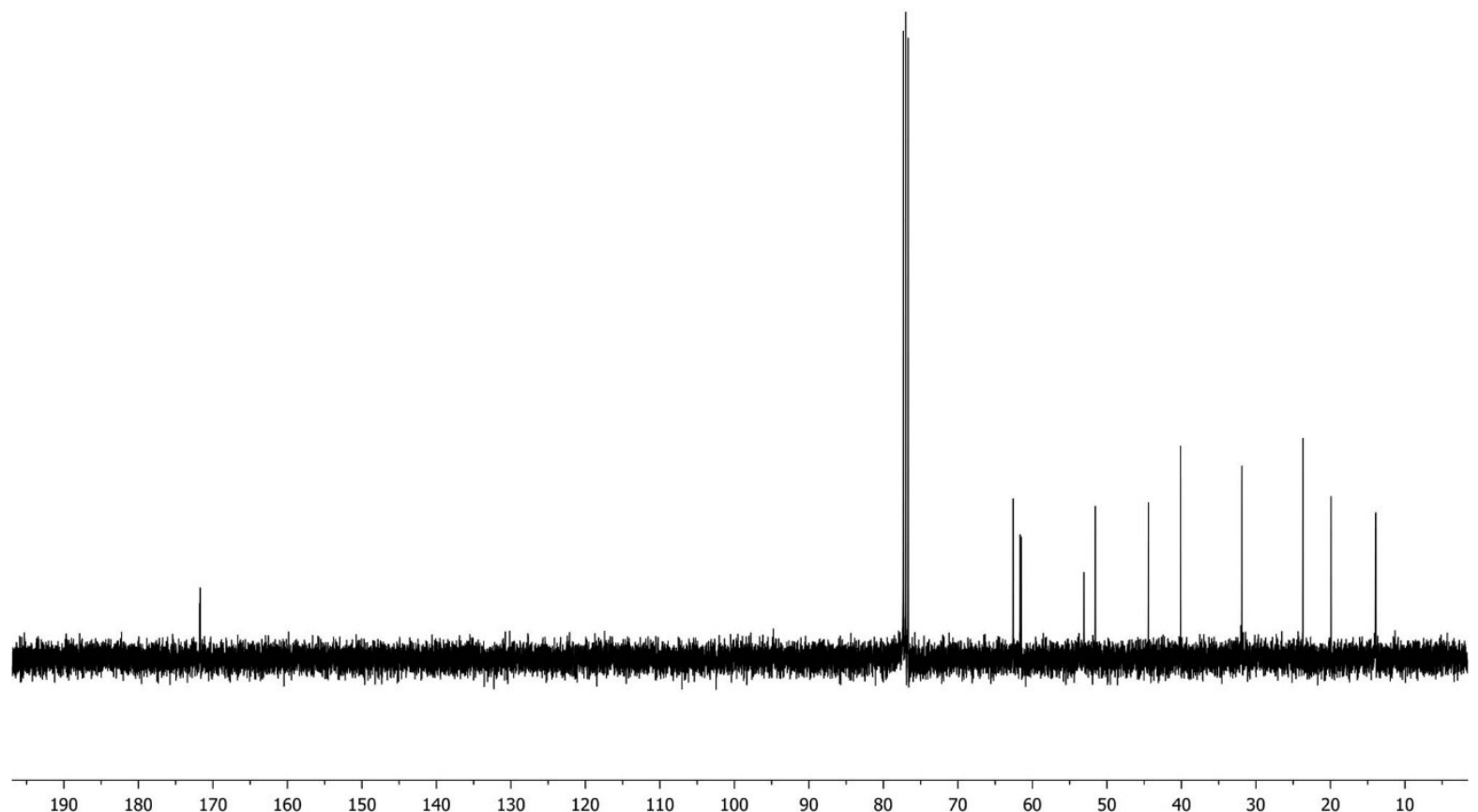


$$
\int_{\text {EtOOC }}^{\mathrm{Br}} \int_{\mathrm{Br}}^{\mathrm{Br}}
$$

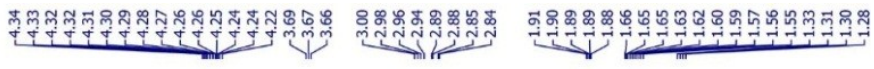

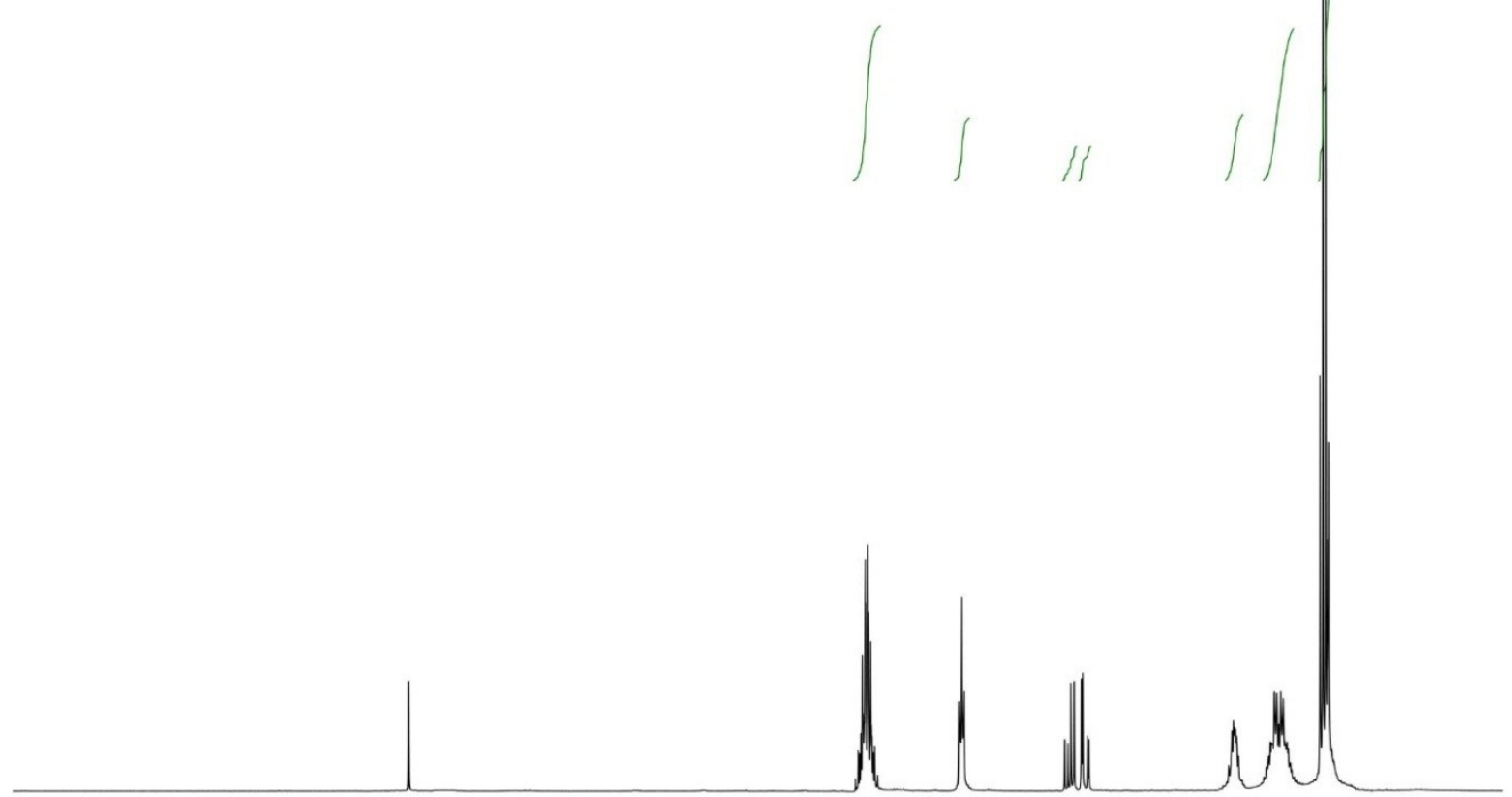

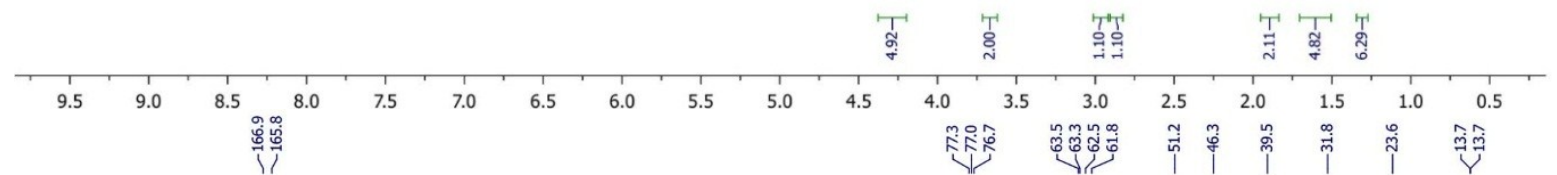

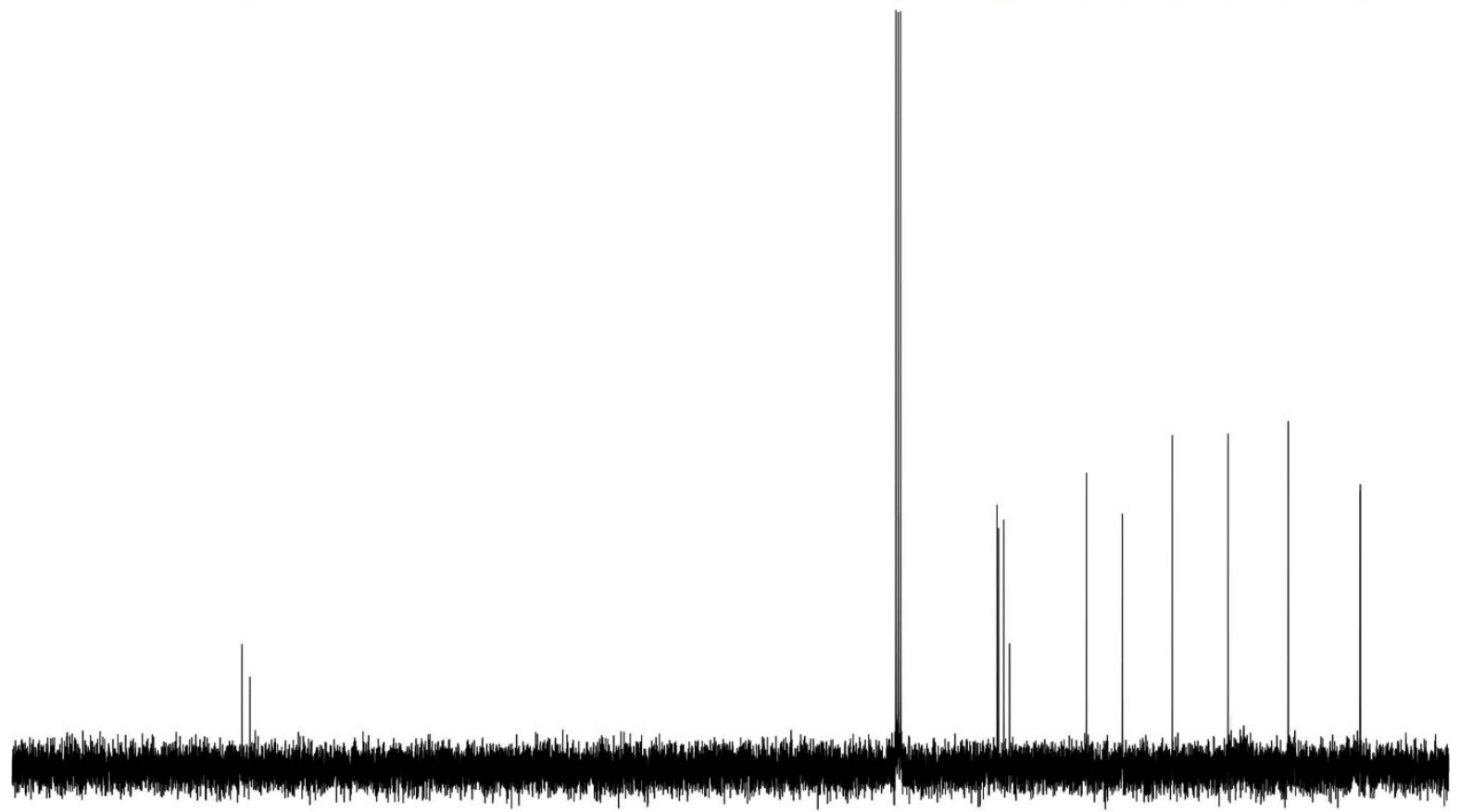

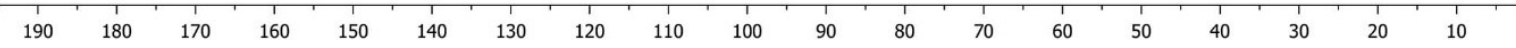




$$
\int_{\int_{F}^{\prime \prime F}}^{\mathrm{Br}} 4 d
$$

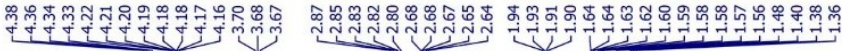
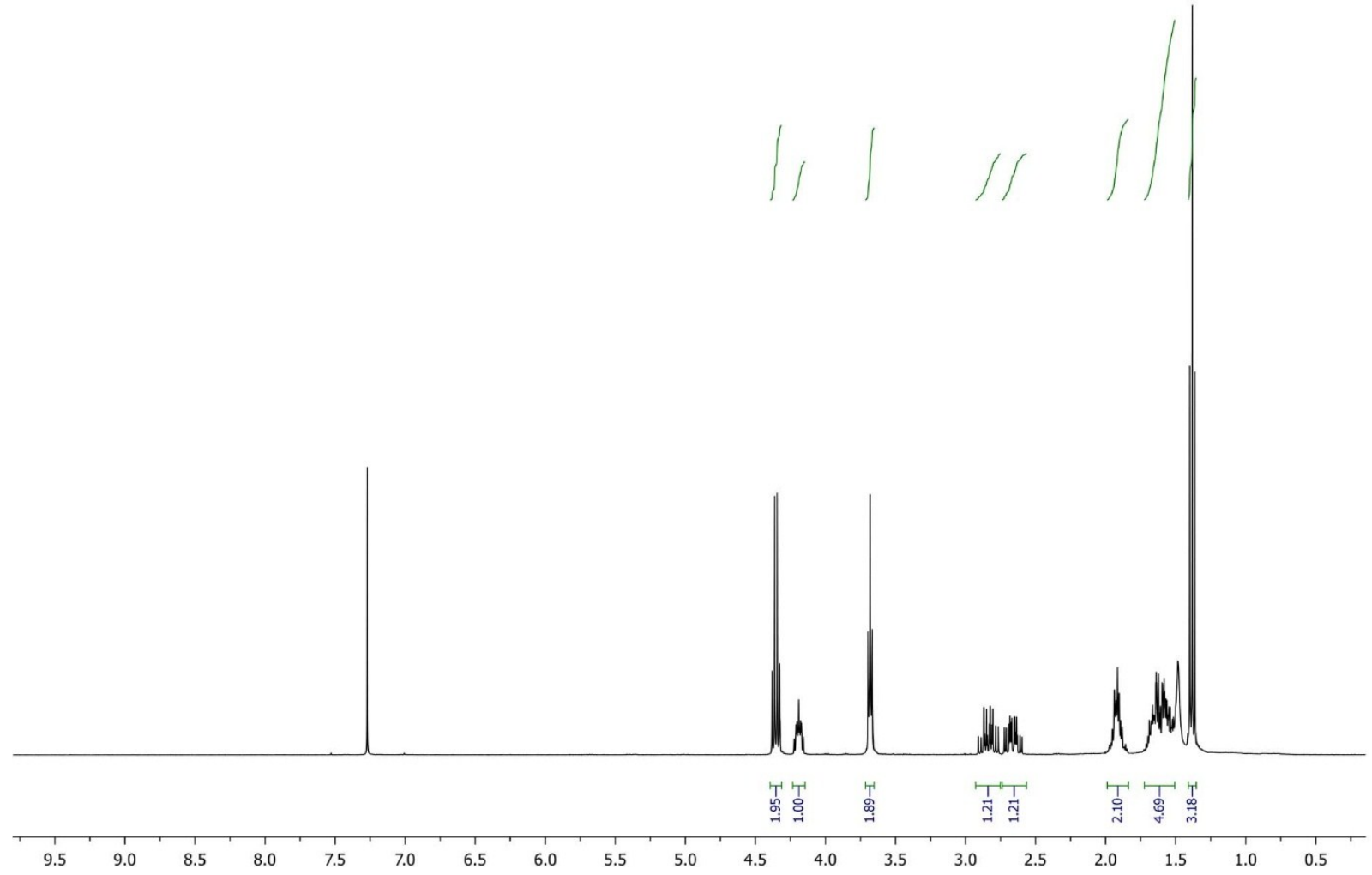

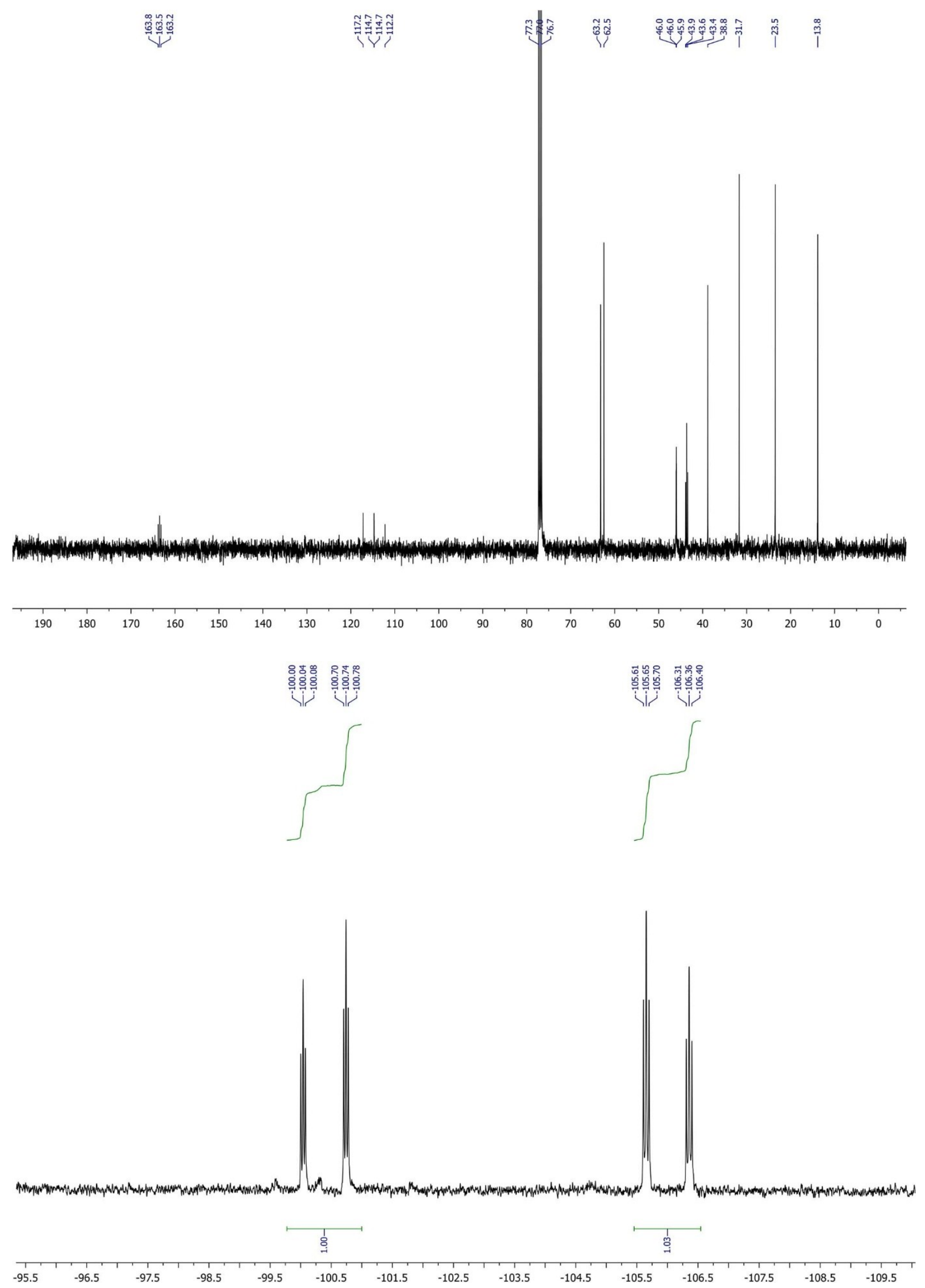
$\int_{\text {Etooc }}^{\mathrm{Br}} \int_{4 \mathbf{F}}^{\mathrm{OH}}$

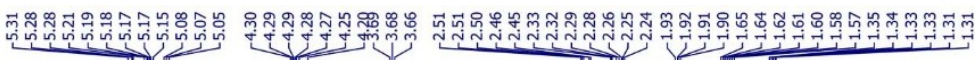

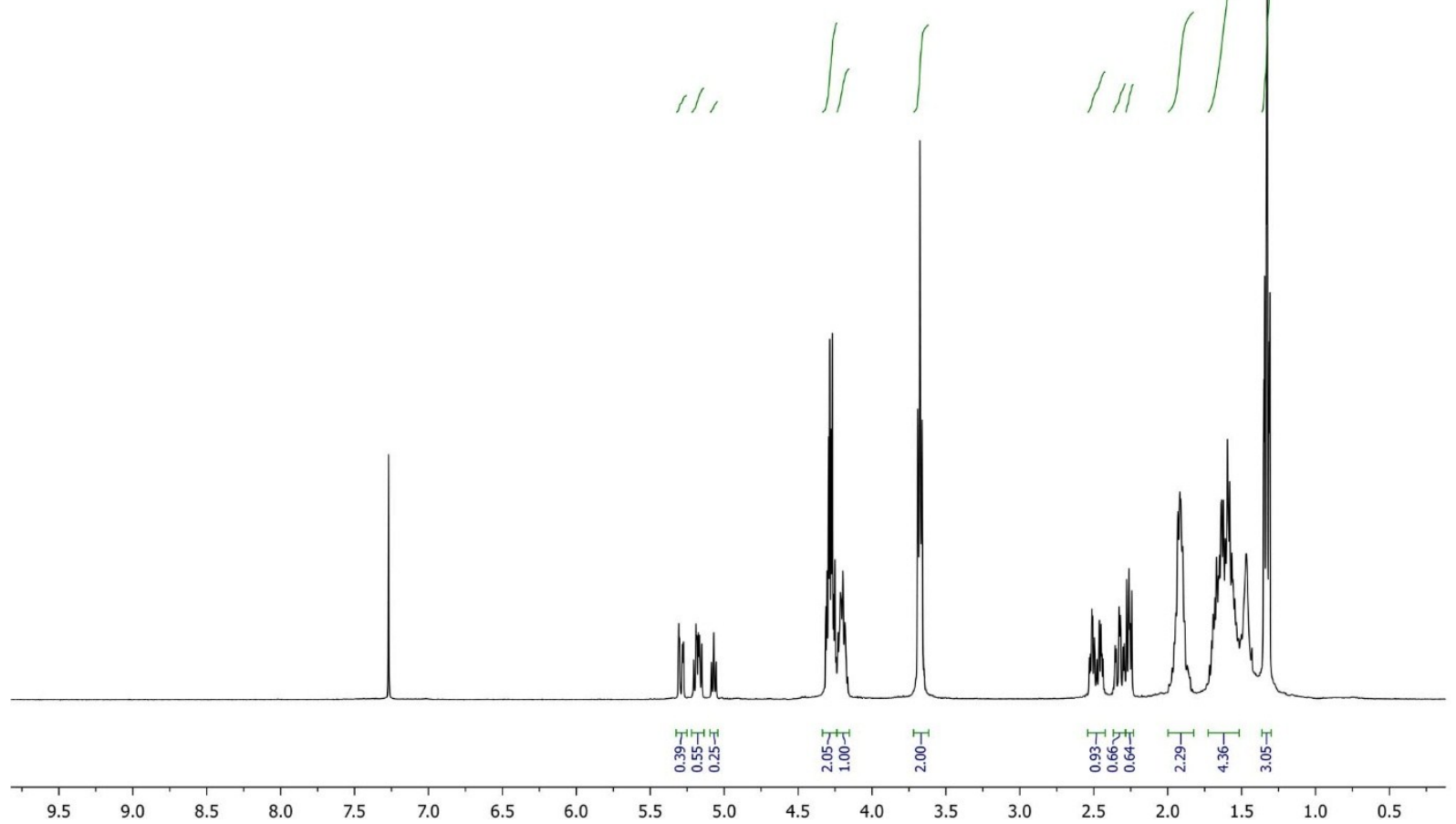




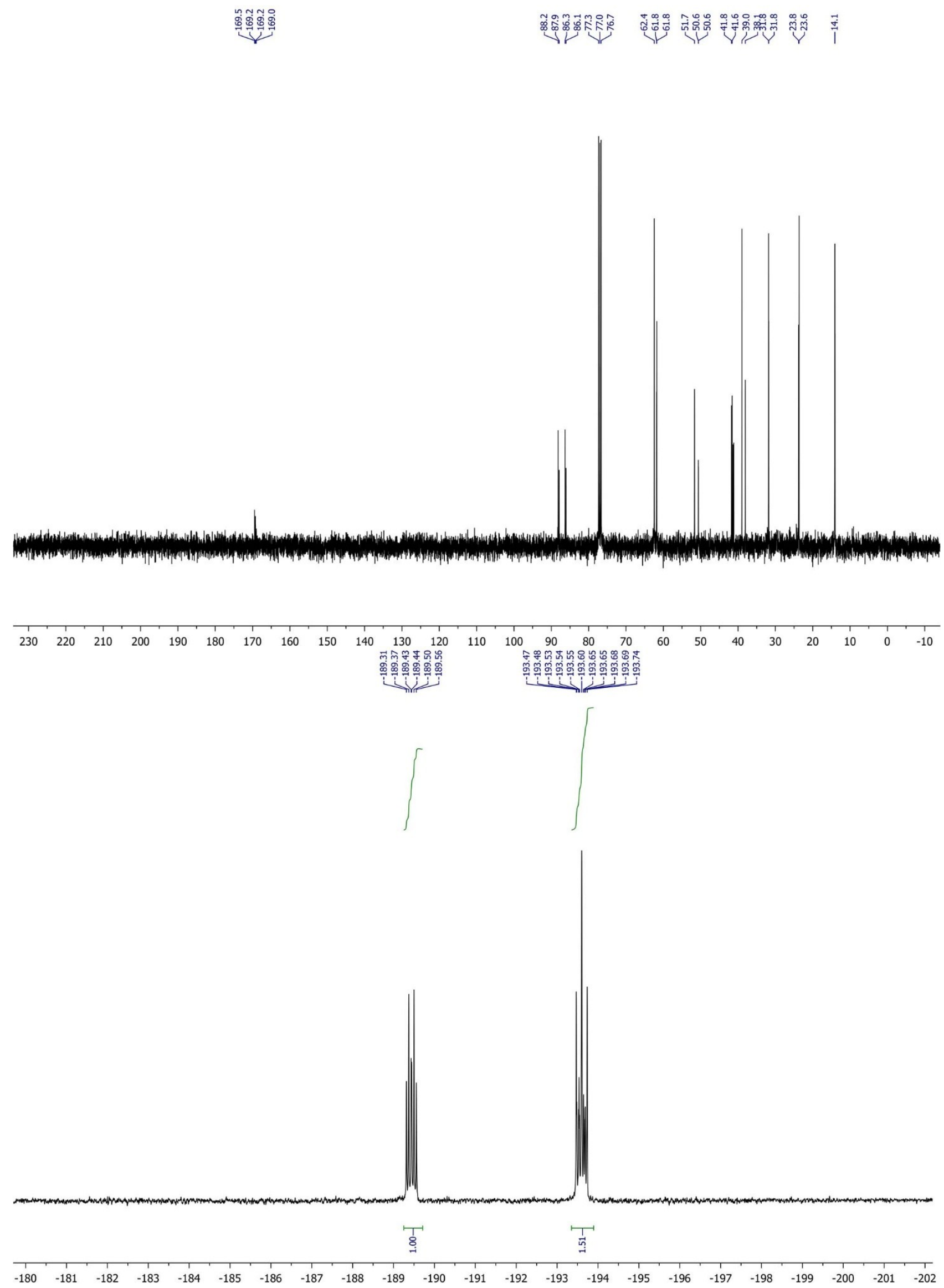


$\int_{\text {Etooc }}^{\mathrm{Br}} \int_{4 \mathbf{f}}$

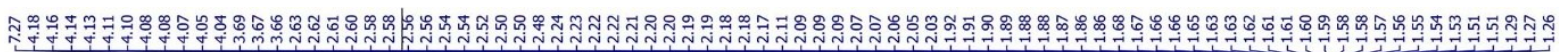

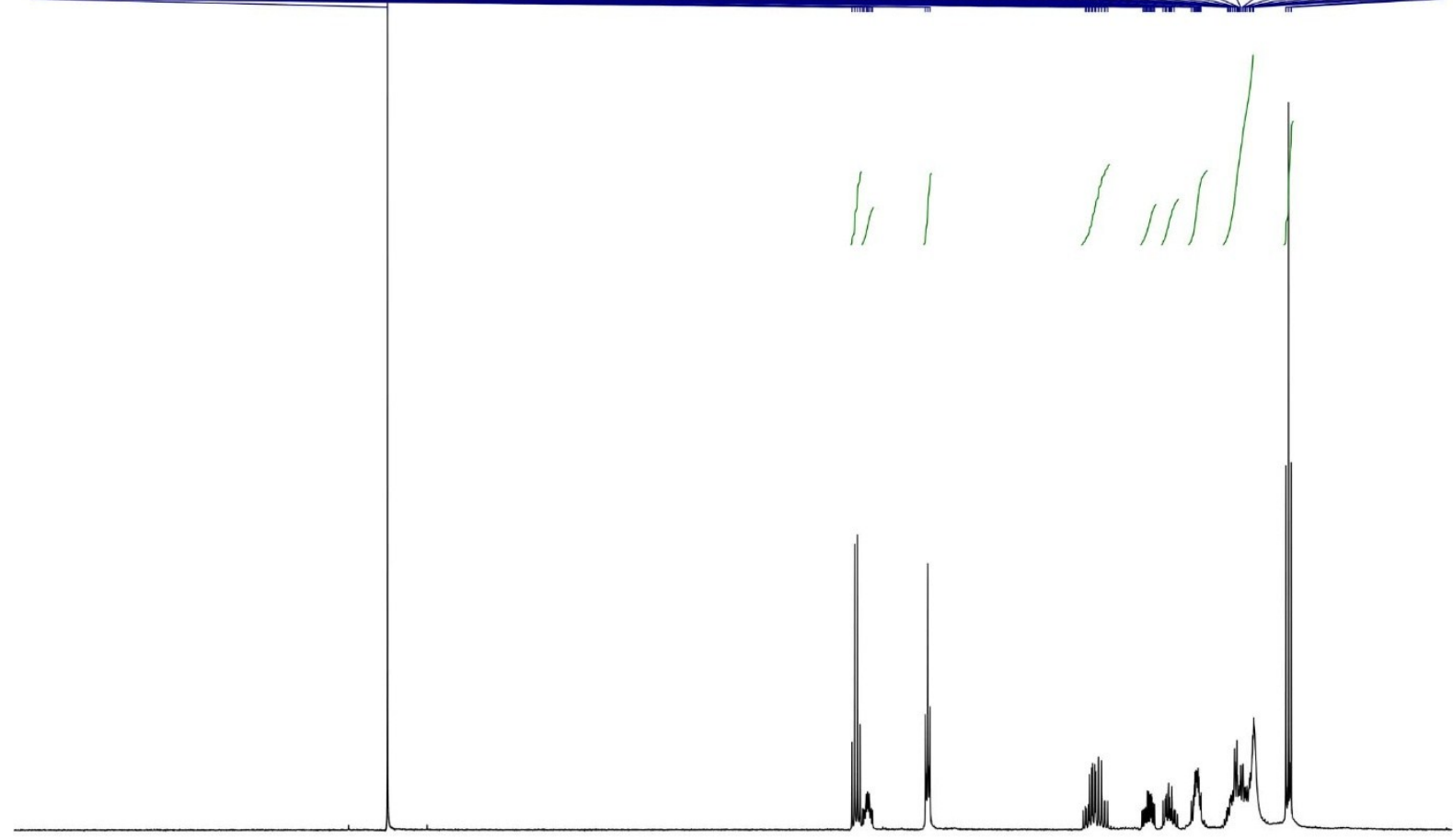

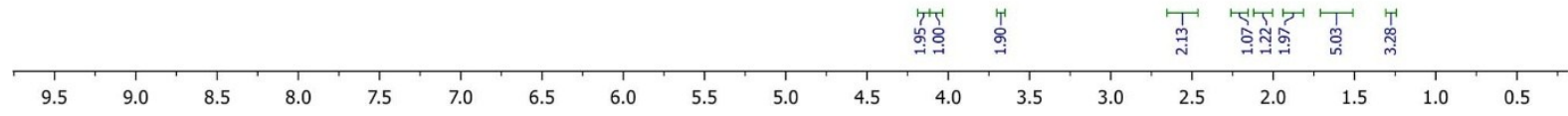

\section{$\stackrel{\infty}{i}$}

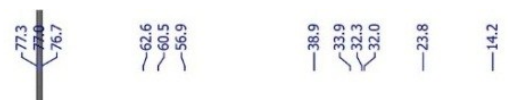

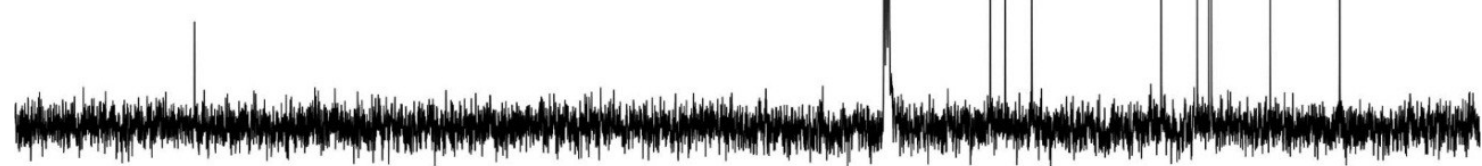

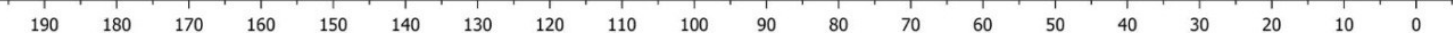


$\int_{\mathrm{CH}_{3}}^{\mathrm{Br}} \mathbf{4 g}$
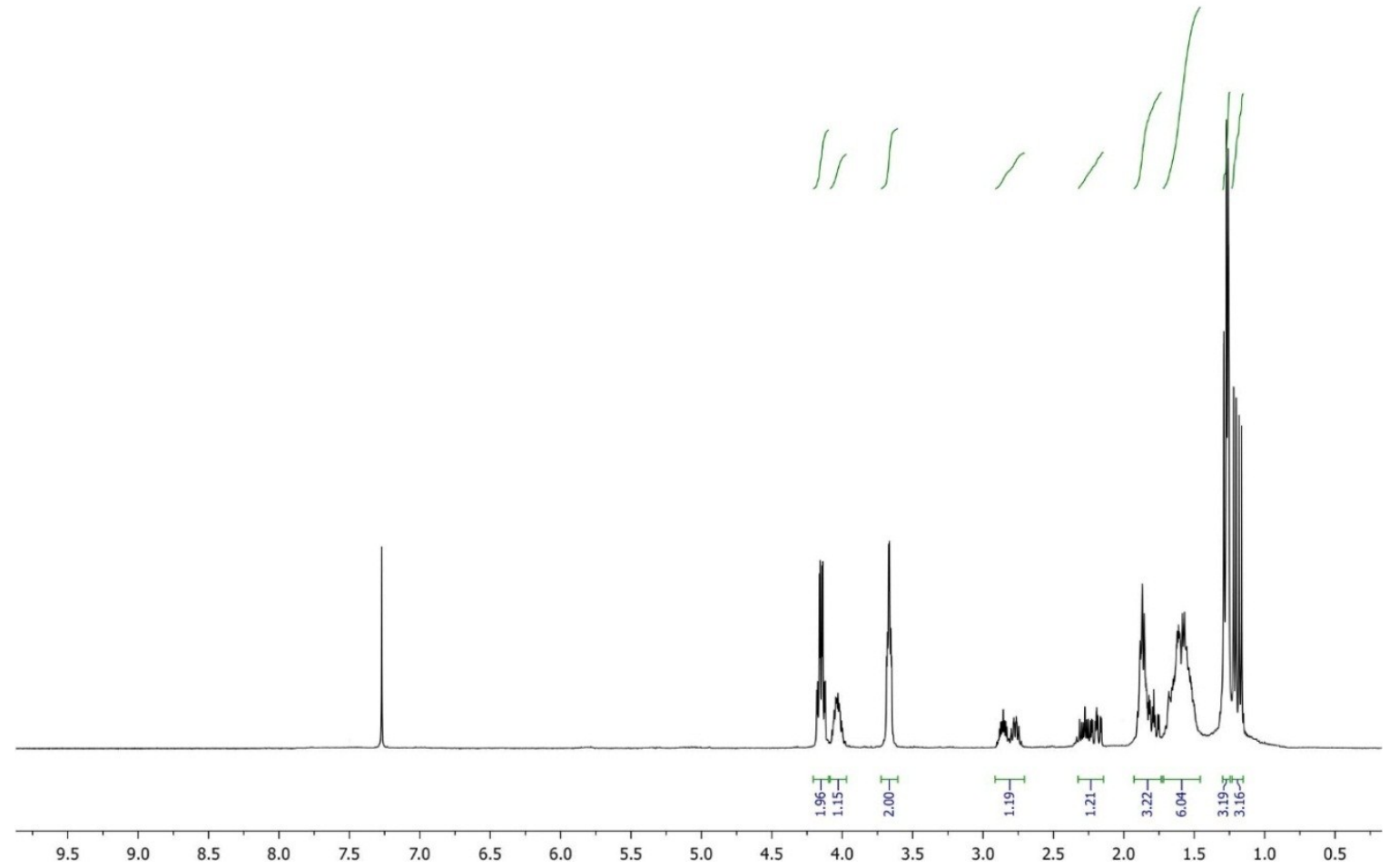

$\stackrel{\circ}{\stackrel{1}{\hat{l}}}$

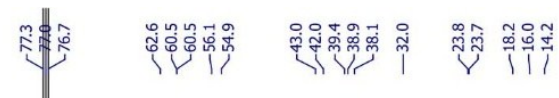

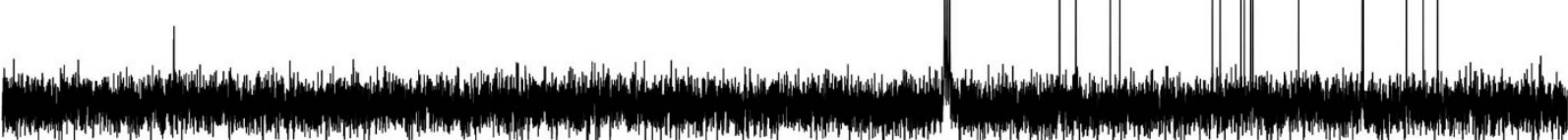

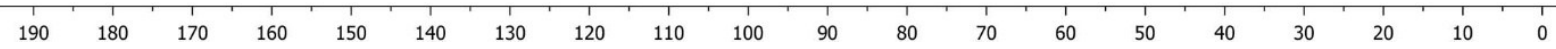


$\int_{N C}^{\mathrm{Br}} \int_{4 h}^{\mathrm{OH}}$

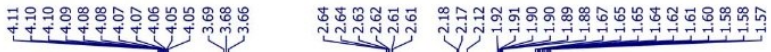
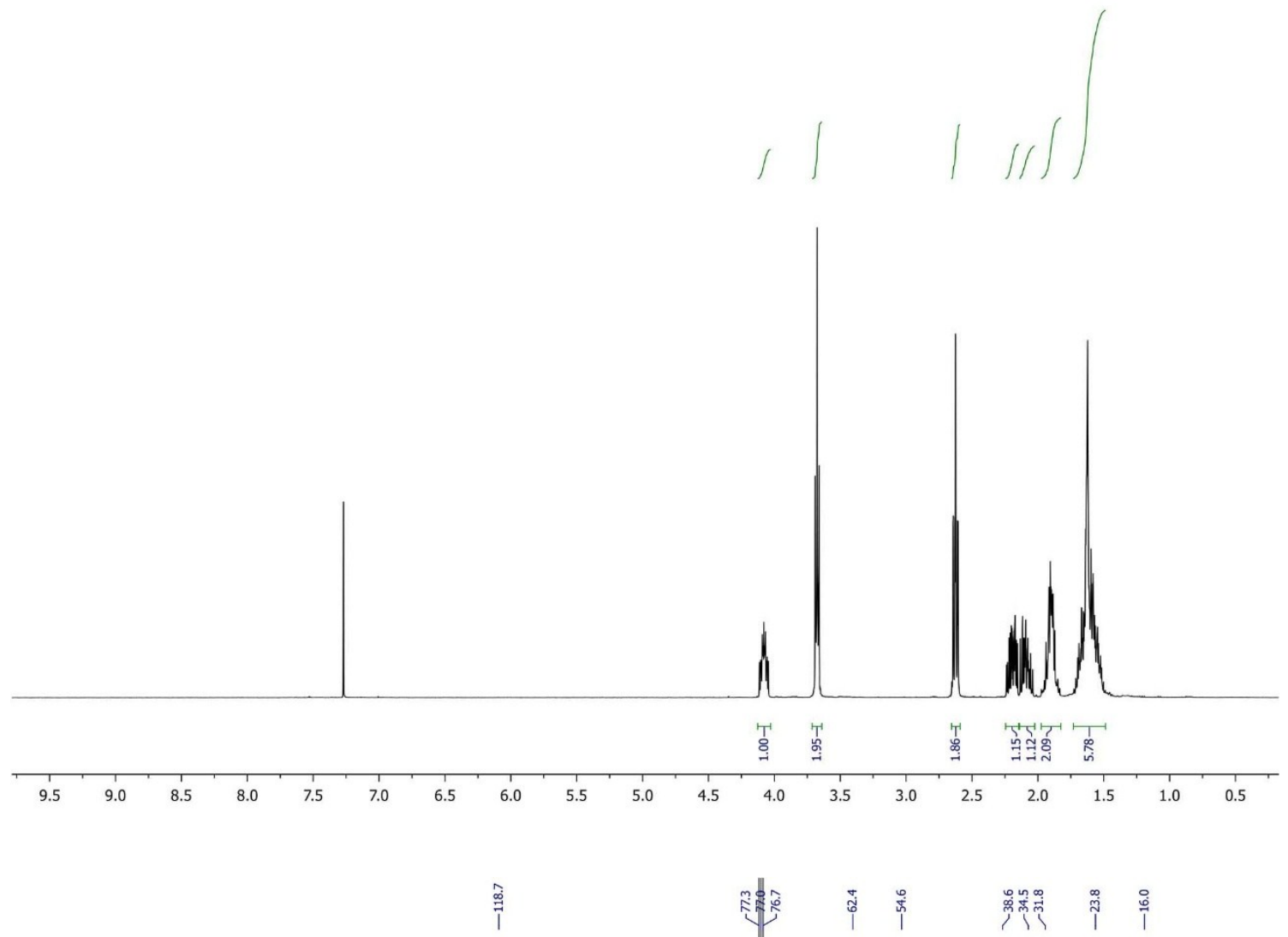

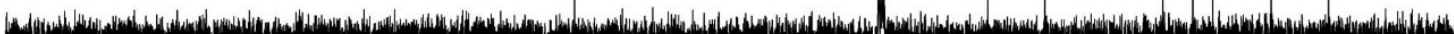

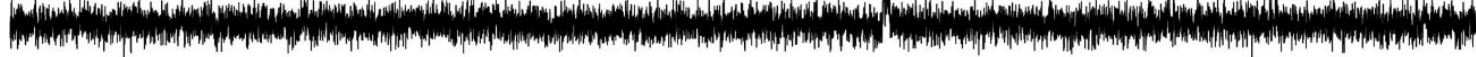

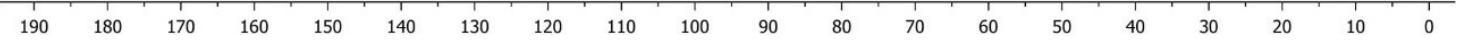


$\int_{\mathrm{CCl}_{3}}^{\mathrm{Cl}} \mathrm{Hr}_{4}^{\mathrm{OH}}$

กิ

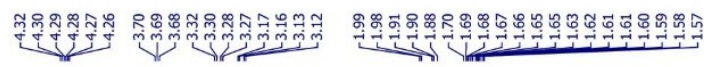

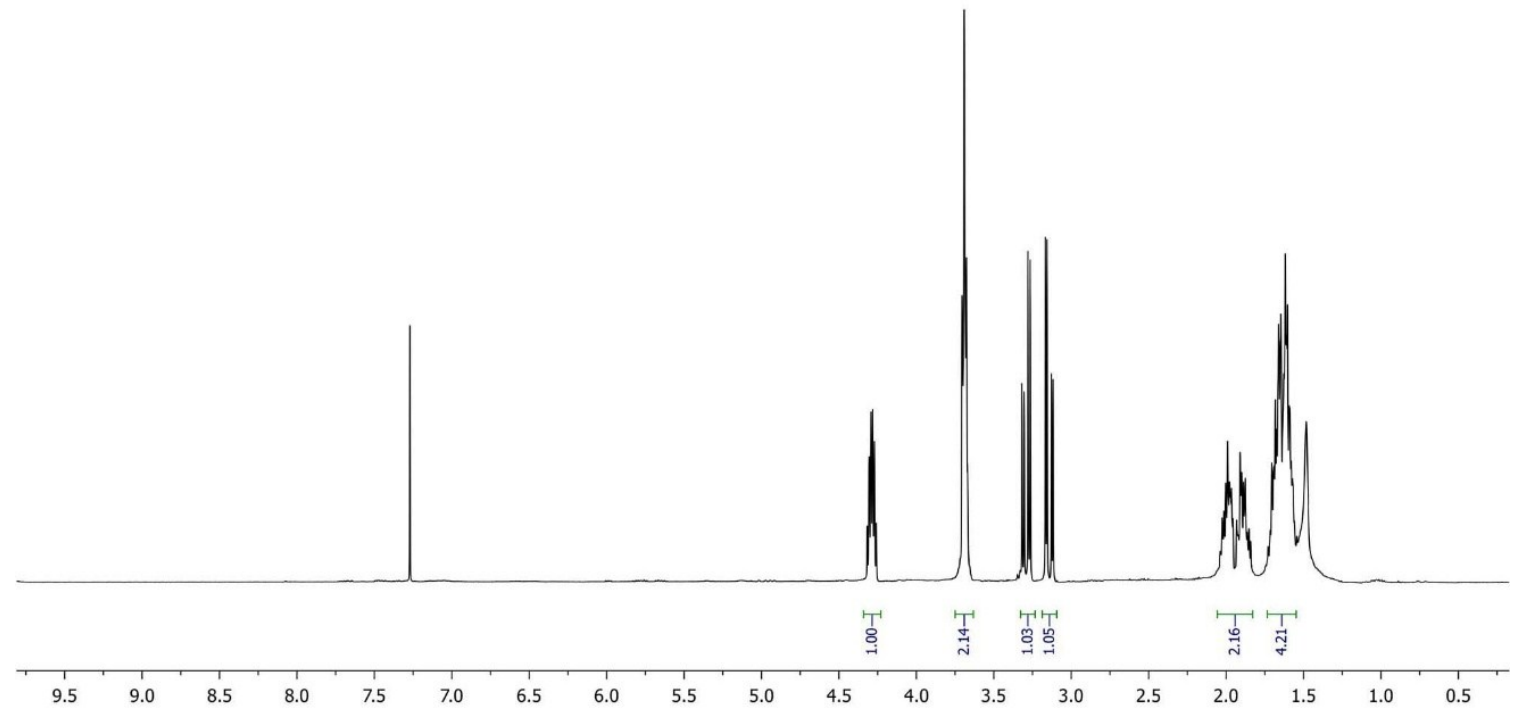

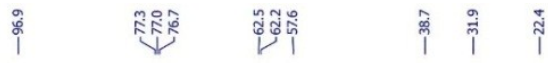

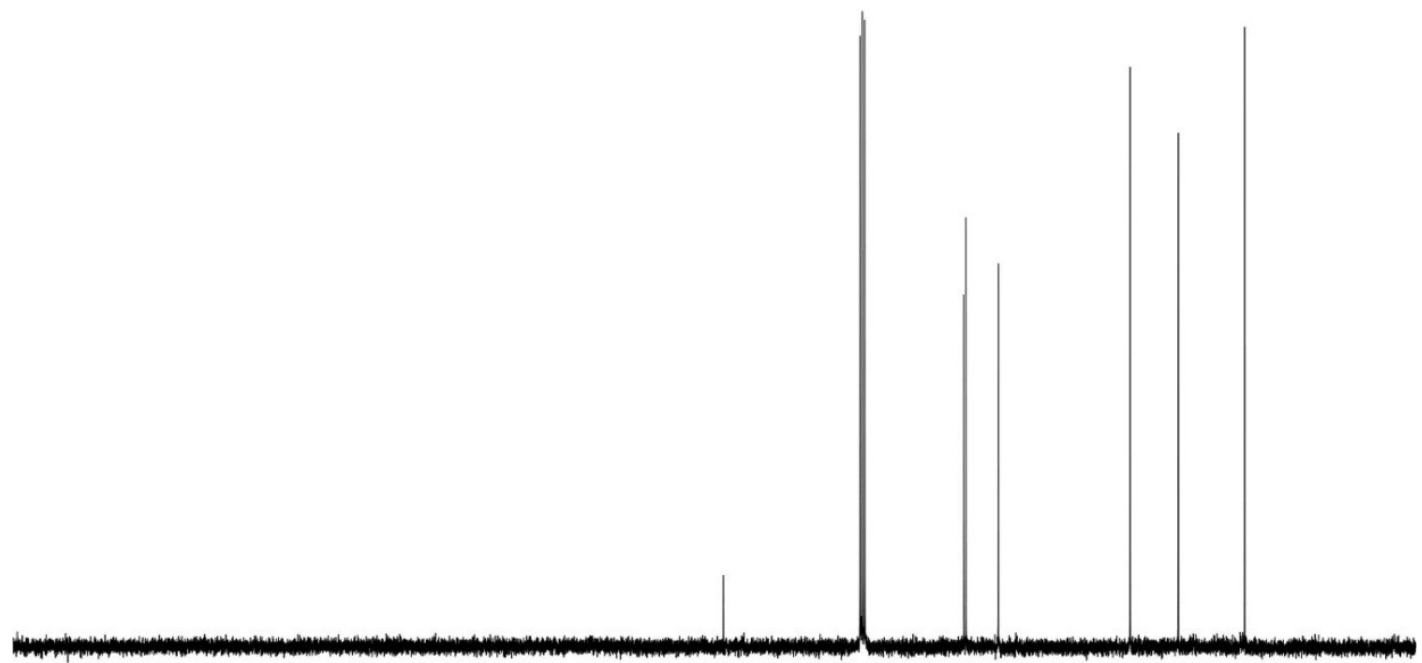

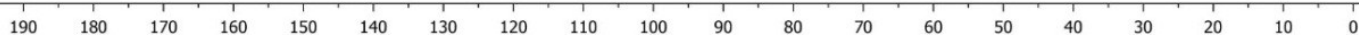


$\int_{\mathrm{Br}^{\mathrm{Br}}}^{\mathrm{Br}} \mathbf{4 j}$

กิ

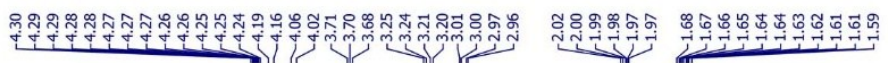

$\left.\operatorname{lig} \int 1\right\}$
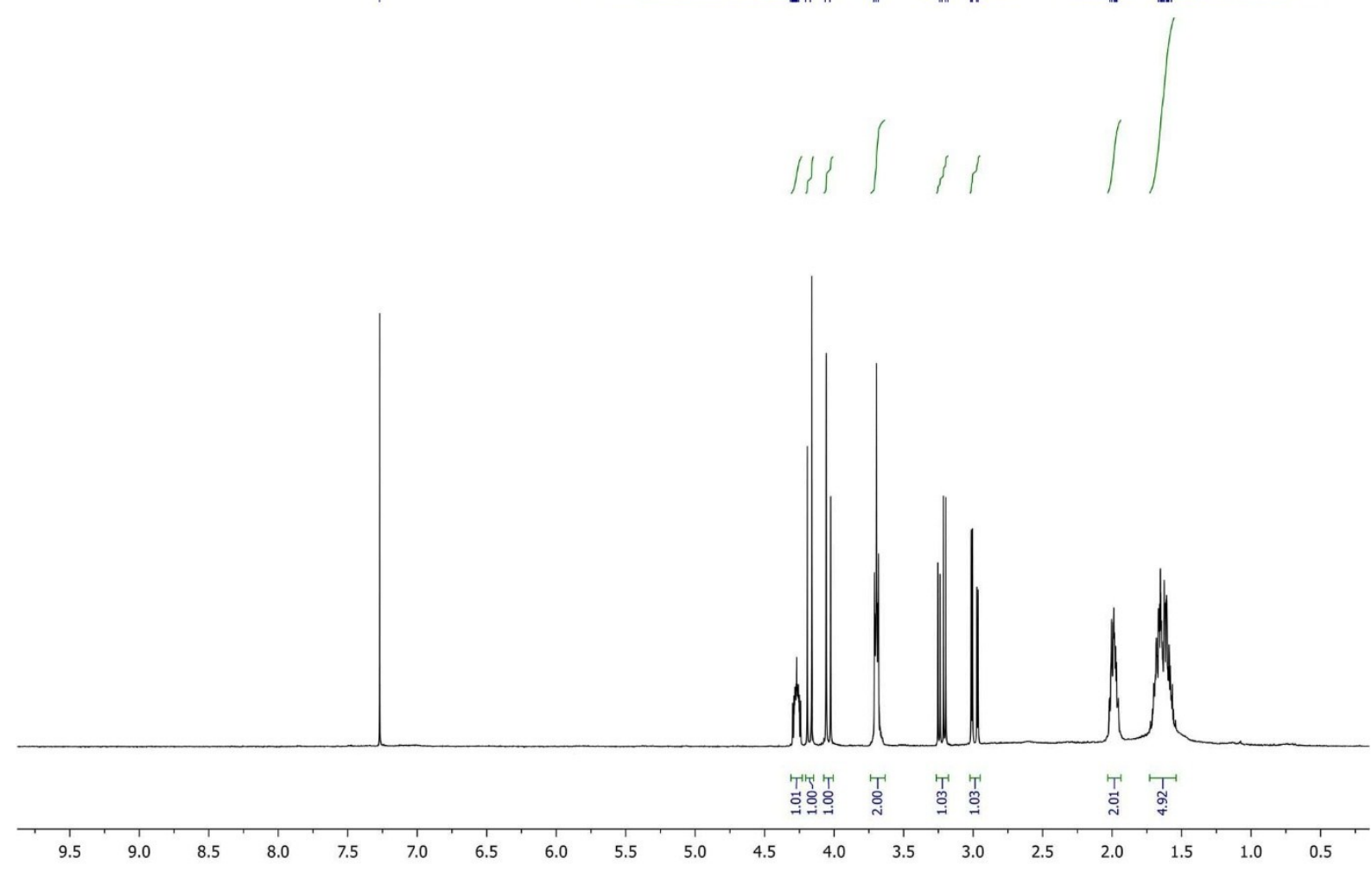

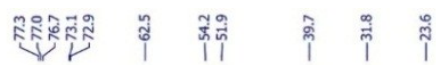
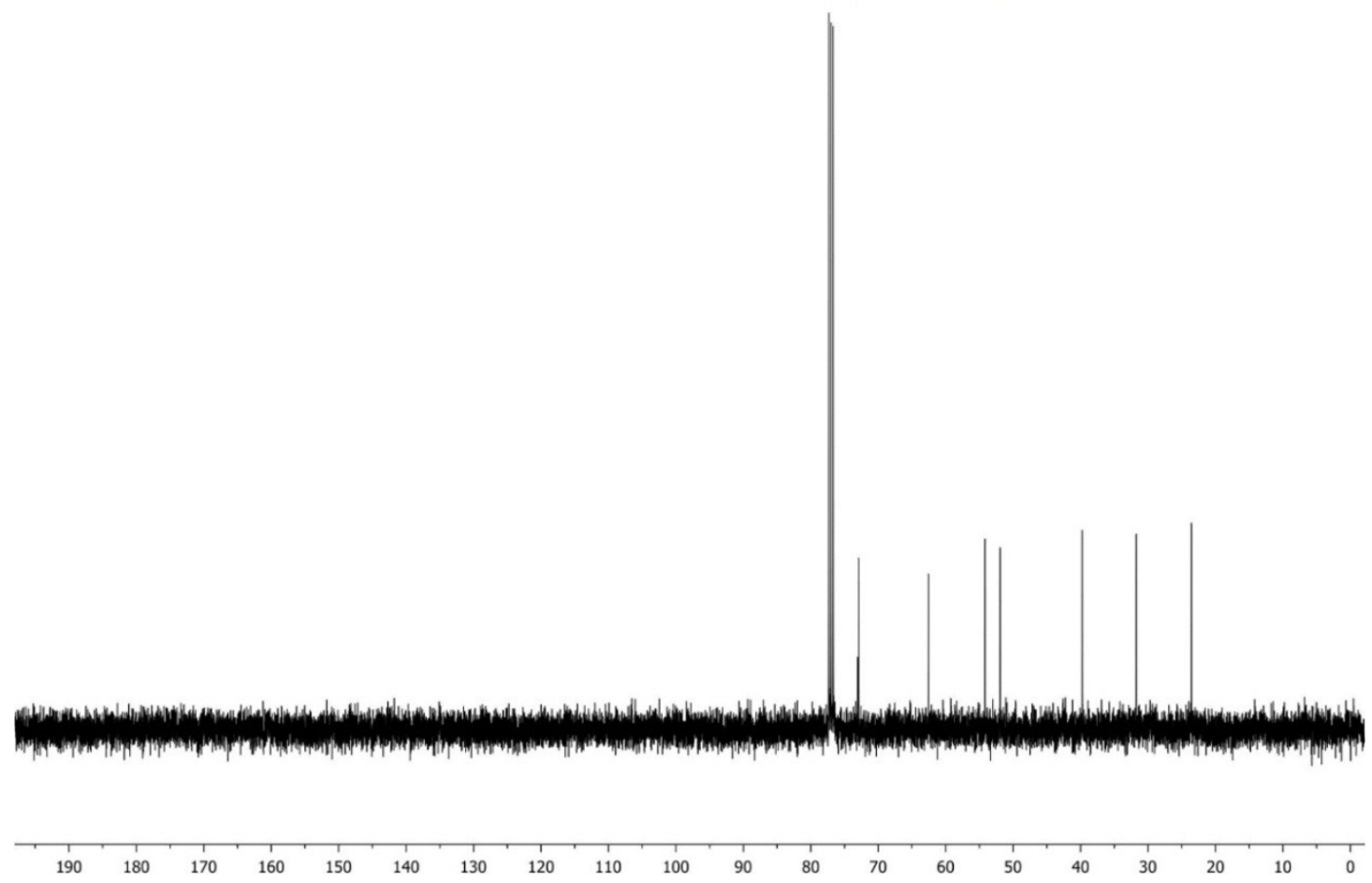
$\mathrm{CF}_{3}\left(\mathrm{CF}_{2}\right)_{4} \mathrm{CF}_{2} \mathrm{I}_{4} \mathrm{OH}_{4 k}$

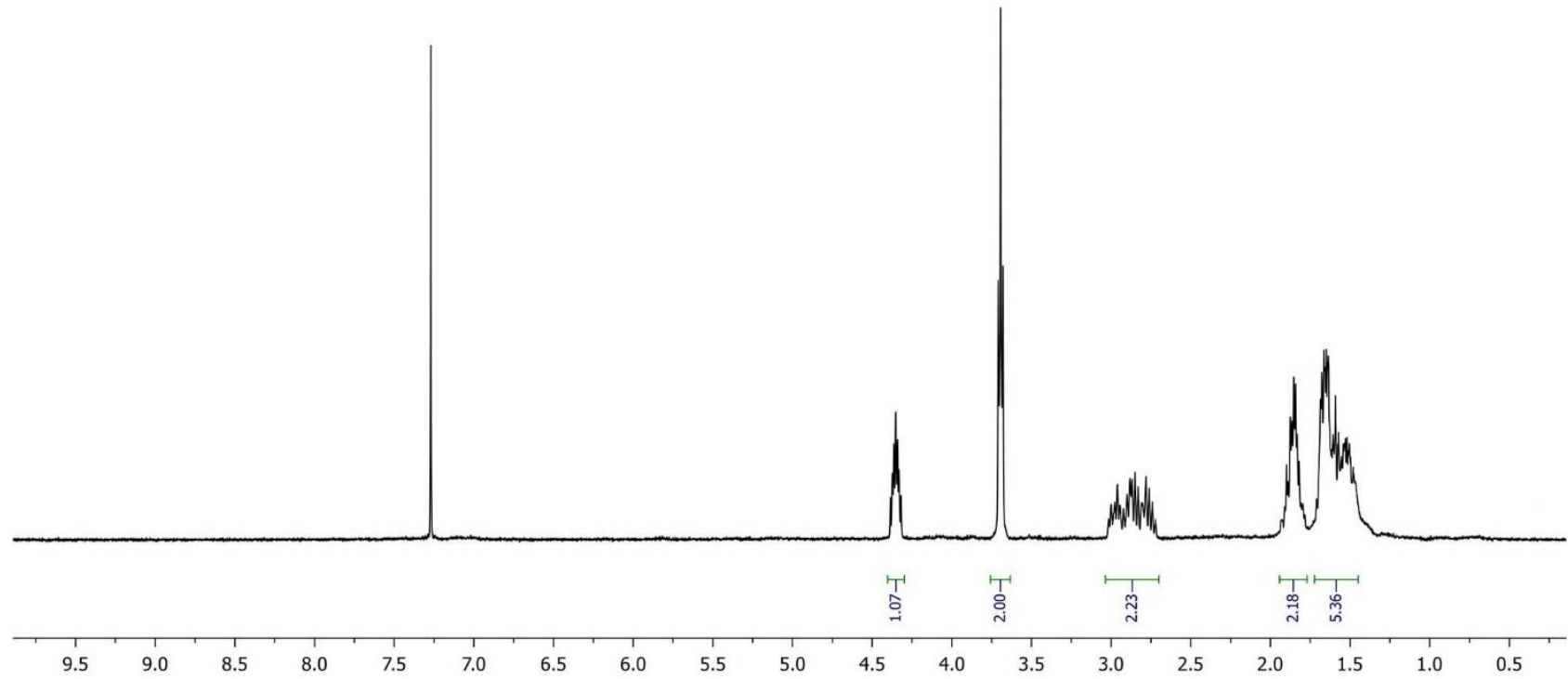



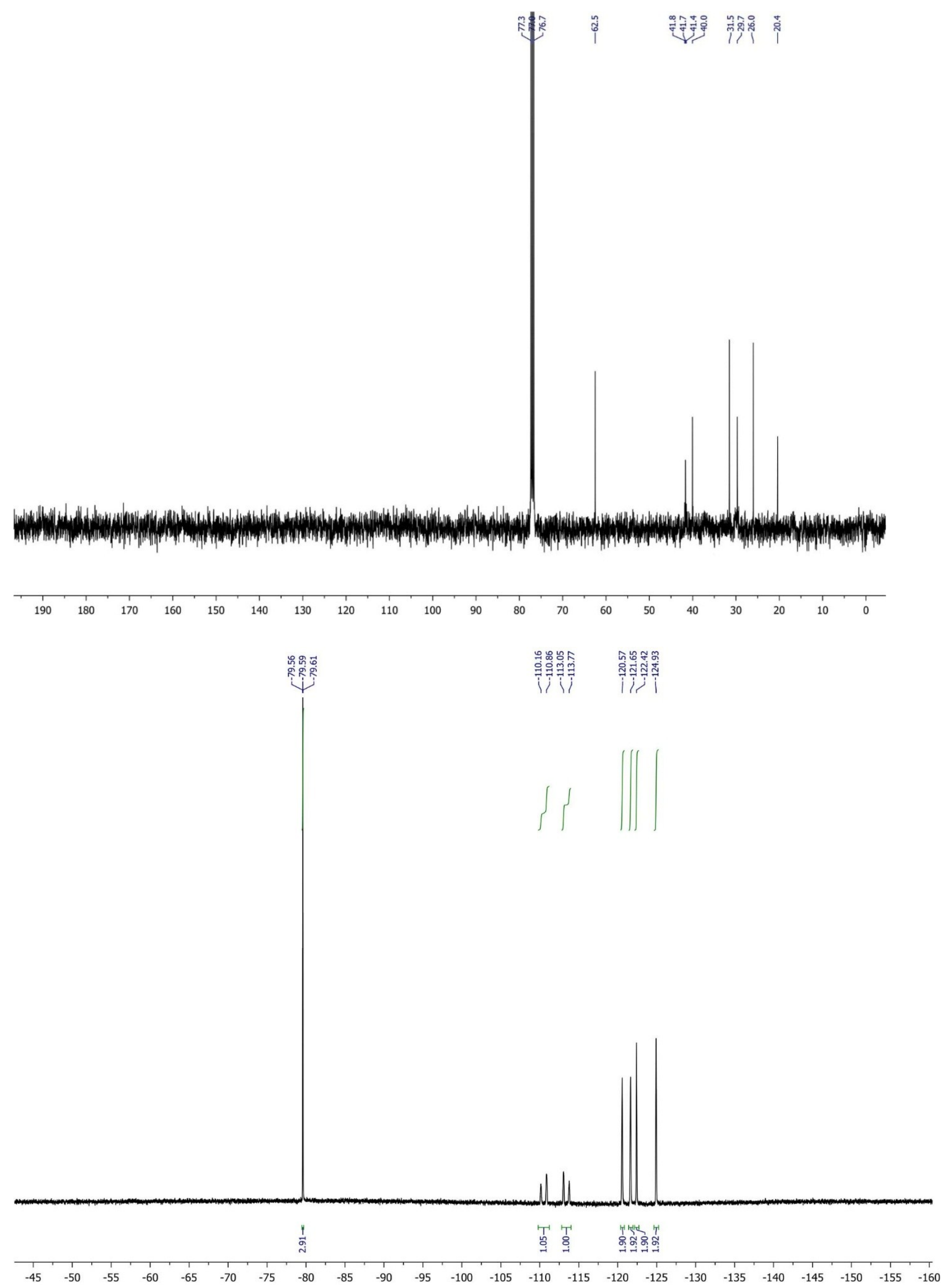


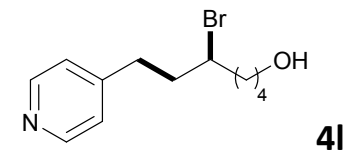
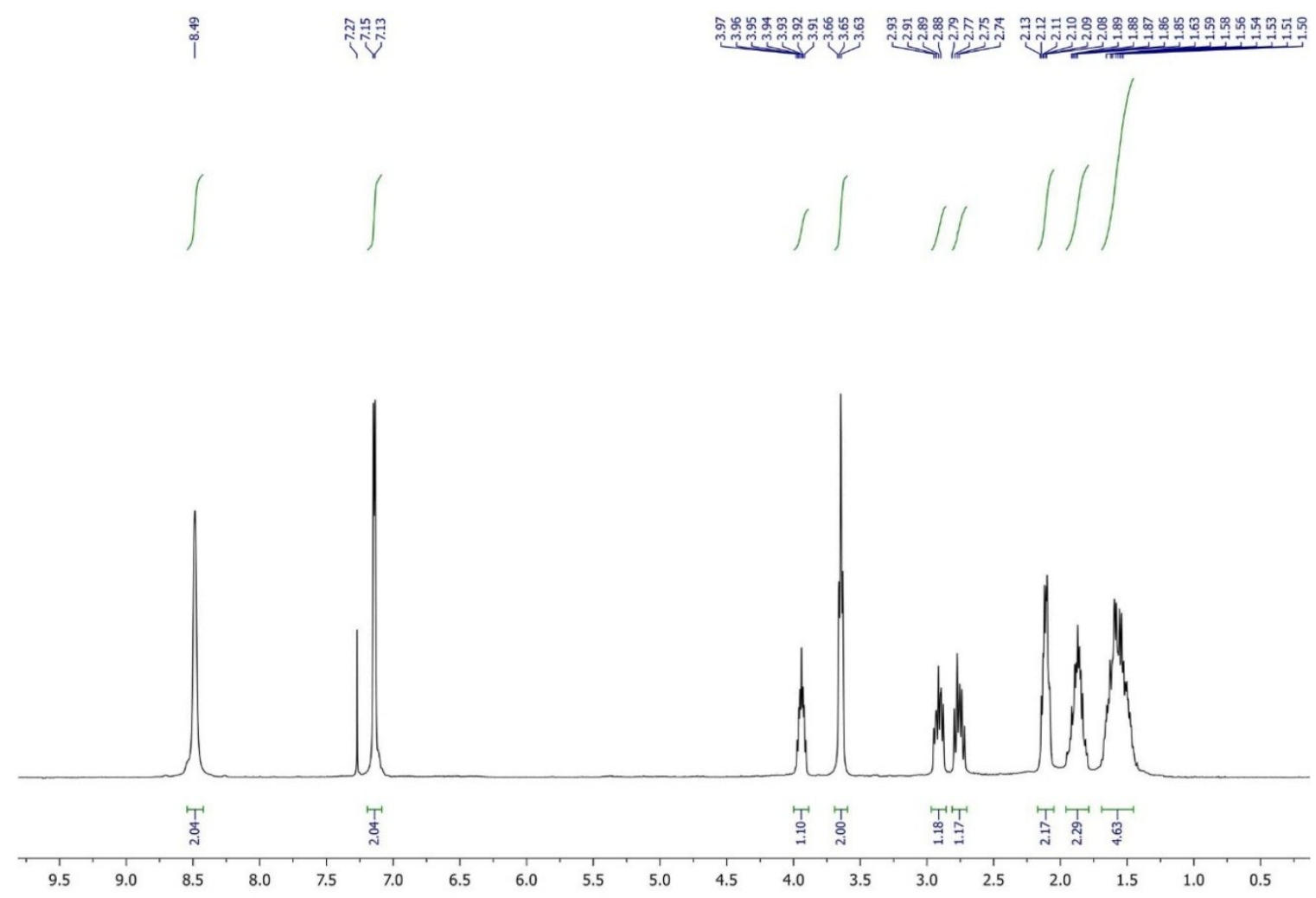

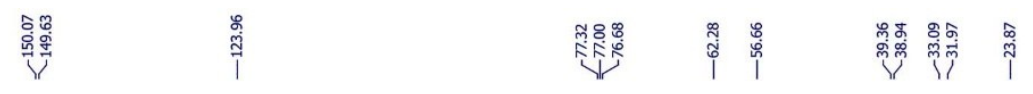

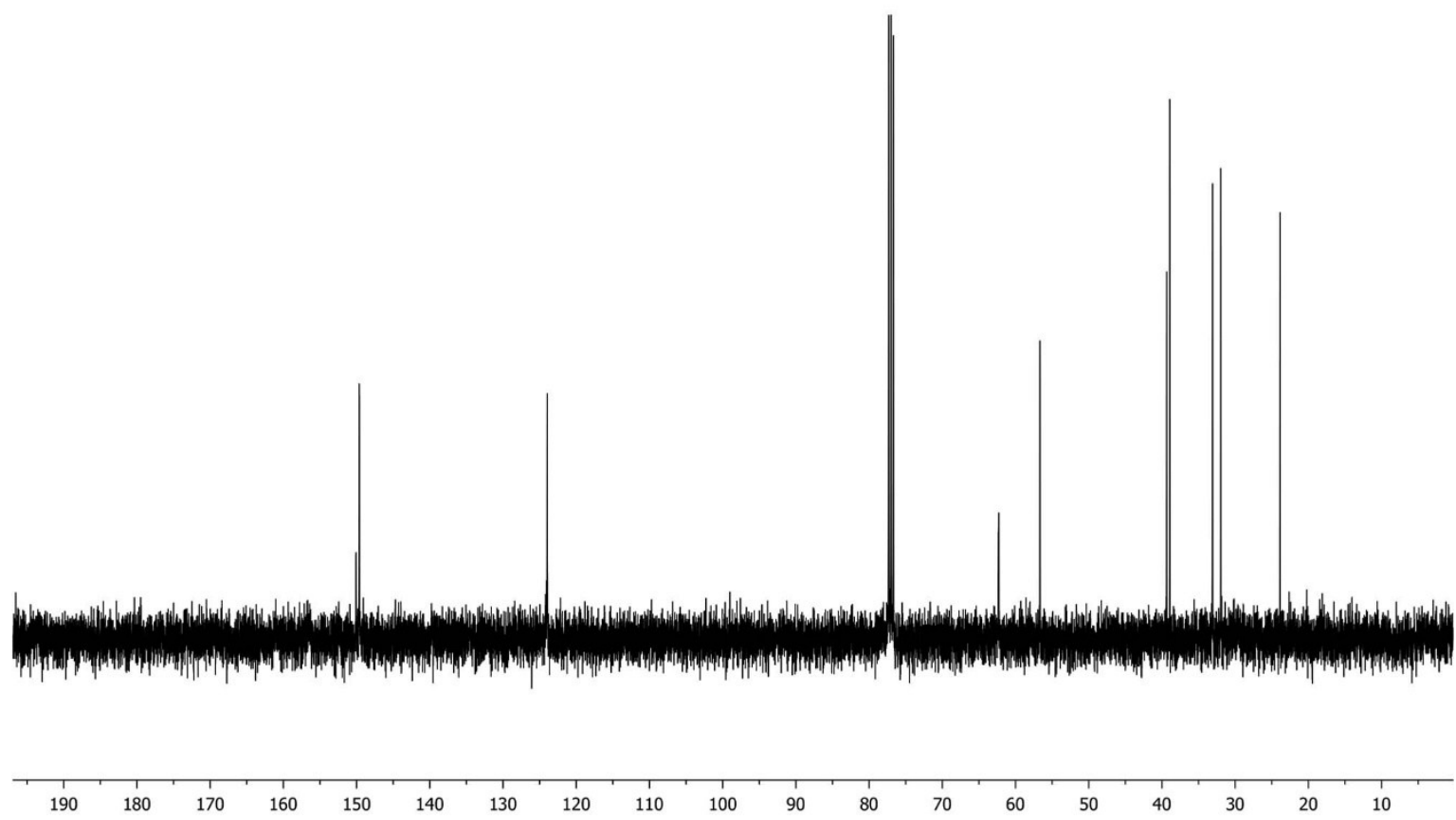


$5 \mathbf{b}$
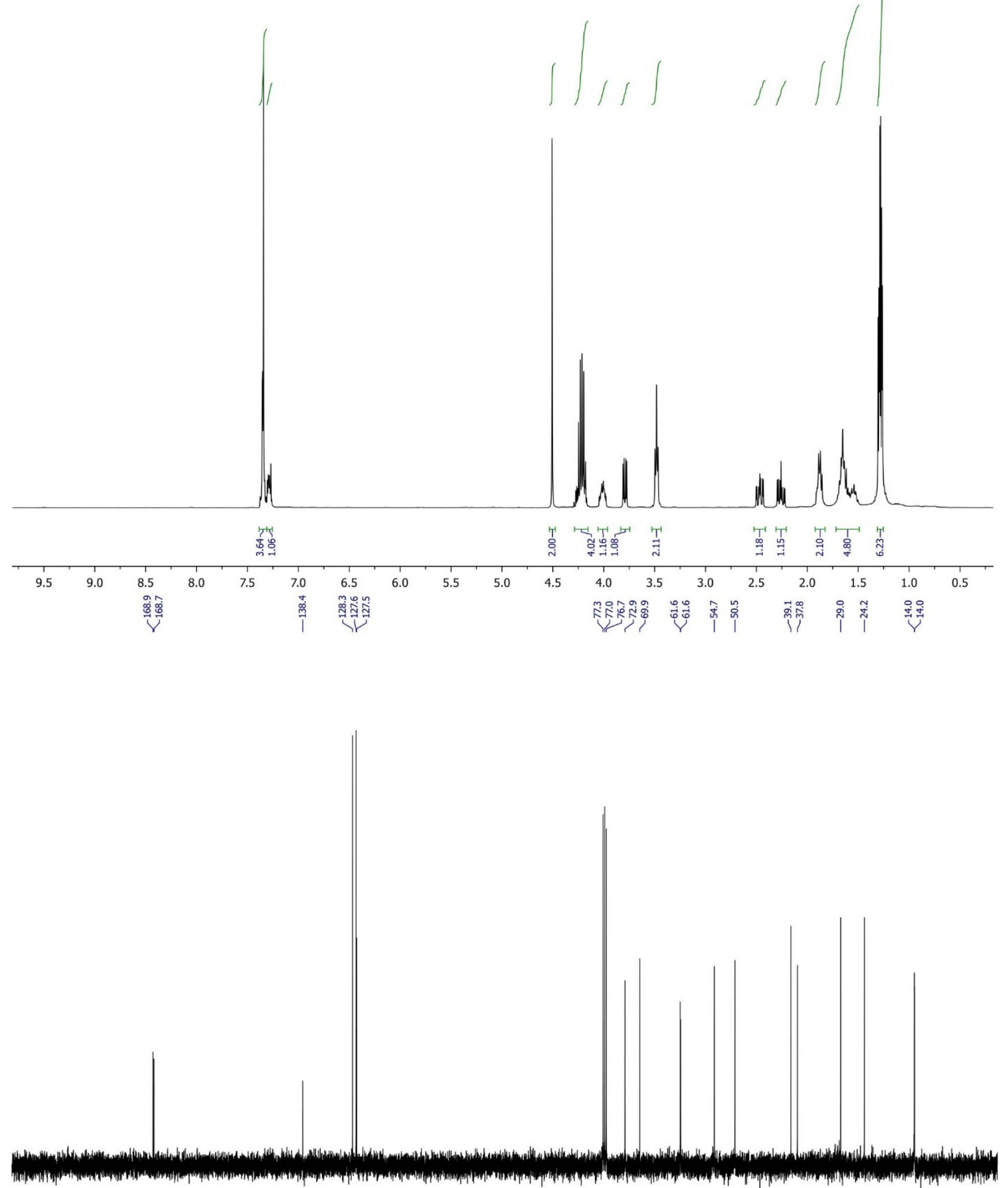

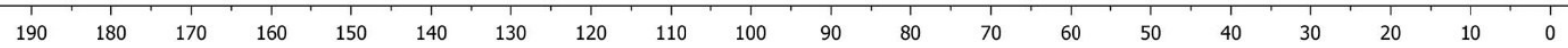




$$
\left.\right|_{\mathbf{B r}} ^{\mathrm{CO}}
$$

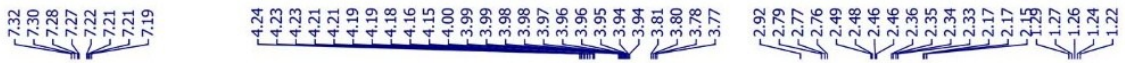<smiles>C#C</smiles><smiles>C1=CCCC=C1</smiles>
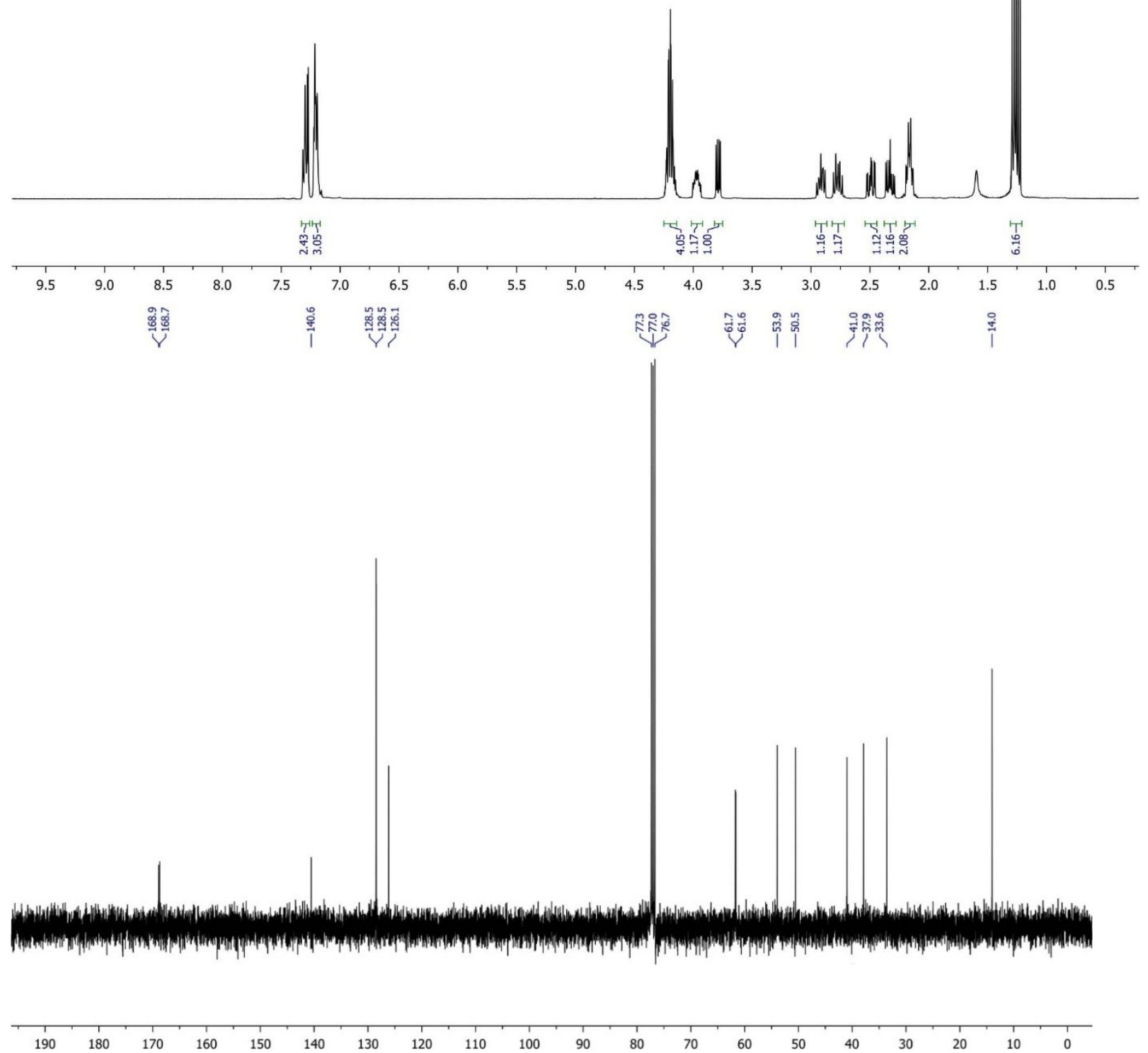
$\prod_{\mathrm{O}}^{\mathrm{BnO}} \overbrace{\mathrm{Br}}^{\mathrm{COOEt}} \mathbf{5 d}$

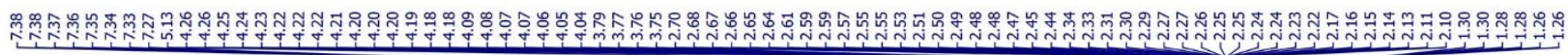
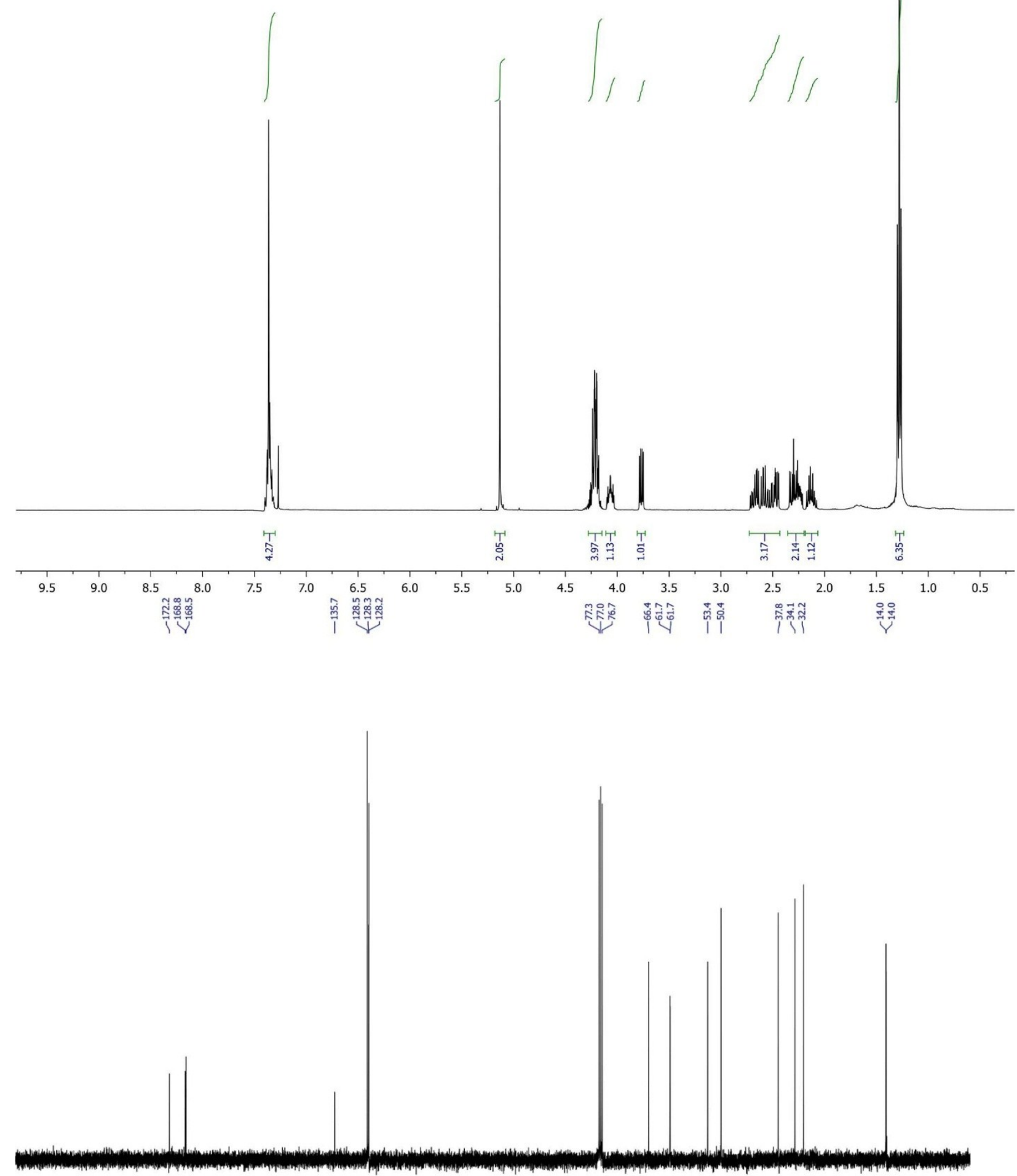

$\begin{array}{llllllllllllllllllllllll}200 & 190 & 180 & 170 & 160 & 150 & 140 & 130 & 120 & 110 & 100 & 90 & 80 & 70 & 60 & 50 & 40 & 30 & 20 & 10 & 0\end{array}$ 
<smiles>CCOC(=O)C(CC(Br)C1CCCCC1)C(=O)OCC</smiles>

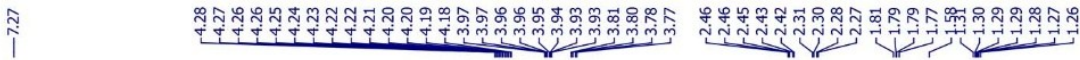
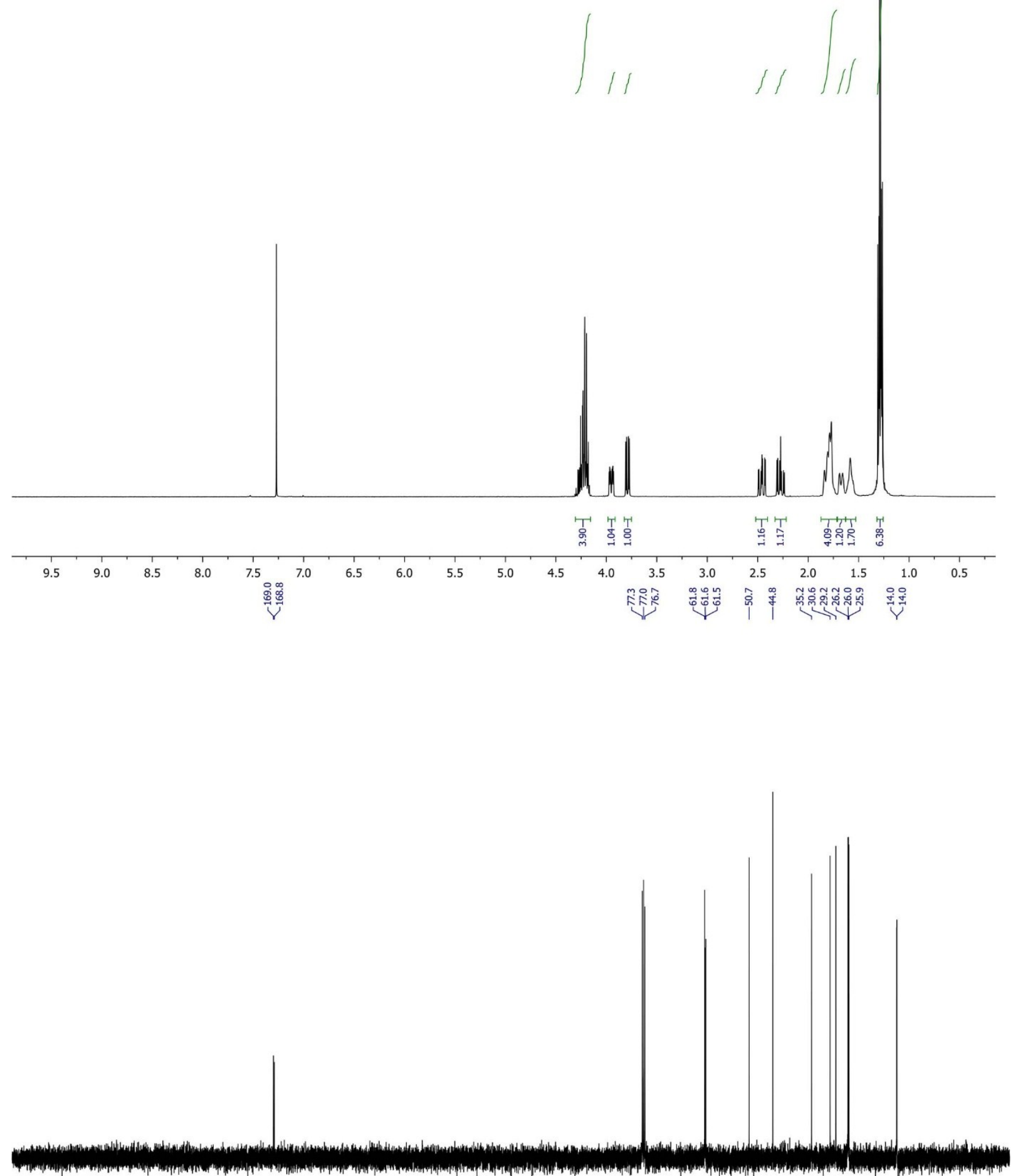

$\begin{array}{llllllllllllllllllllllllll}230 & 220 & 210 & 200 & 190 & 180 & 170 & 160 & 150 & 140 & 130 & 120 & 110 & 100 & 90 & 80 & 70 & 60 & 50 & 40 & 30 & 20 & 10 & 0 & -10\end{array}$ 
$\underbrace{\text { COOEt } \mathbf{5 f}}_{\mathrm{Br}}$

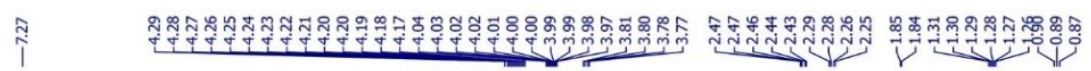
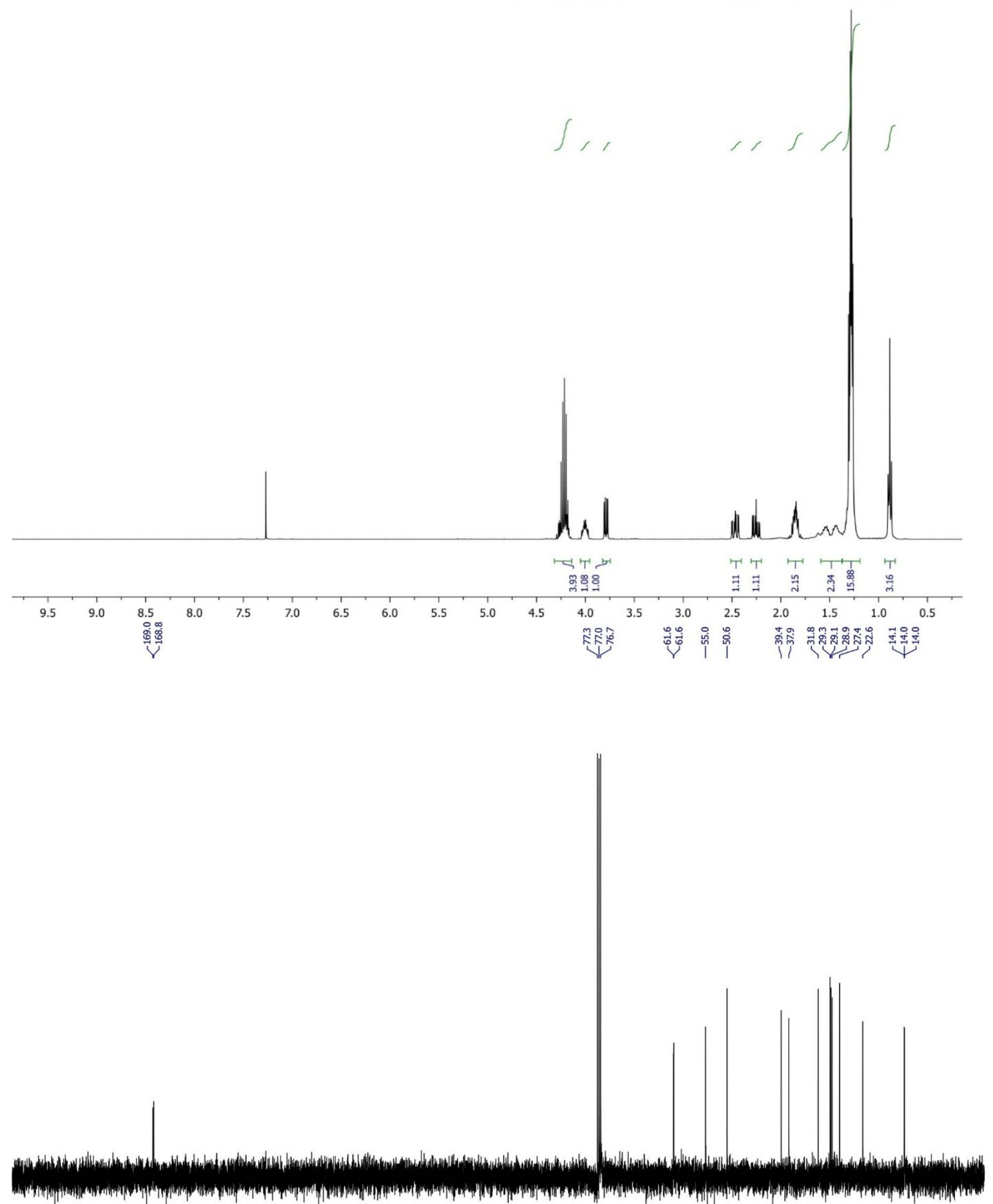

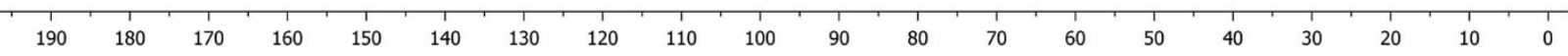




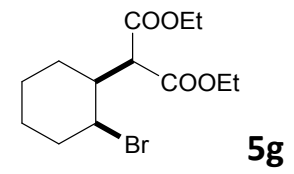

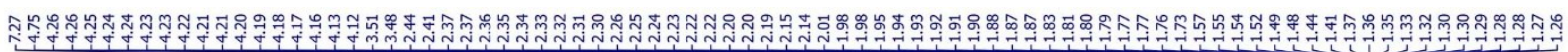

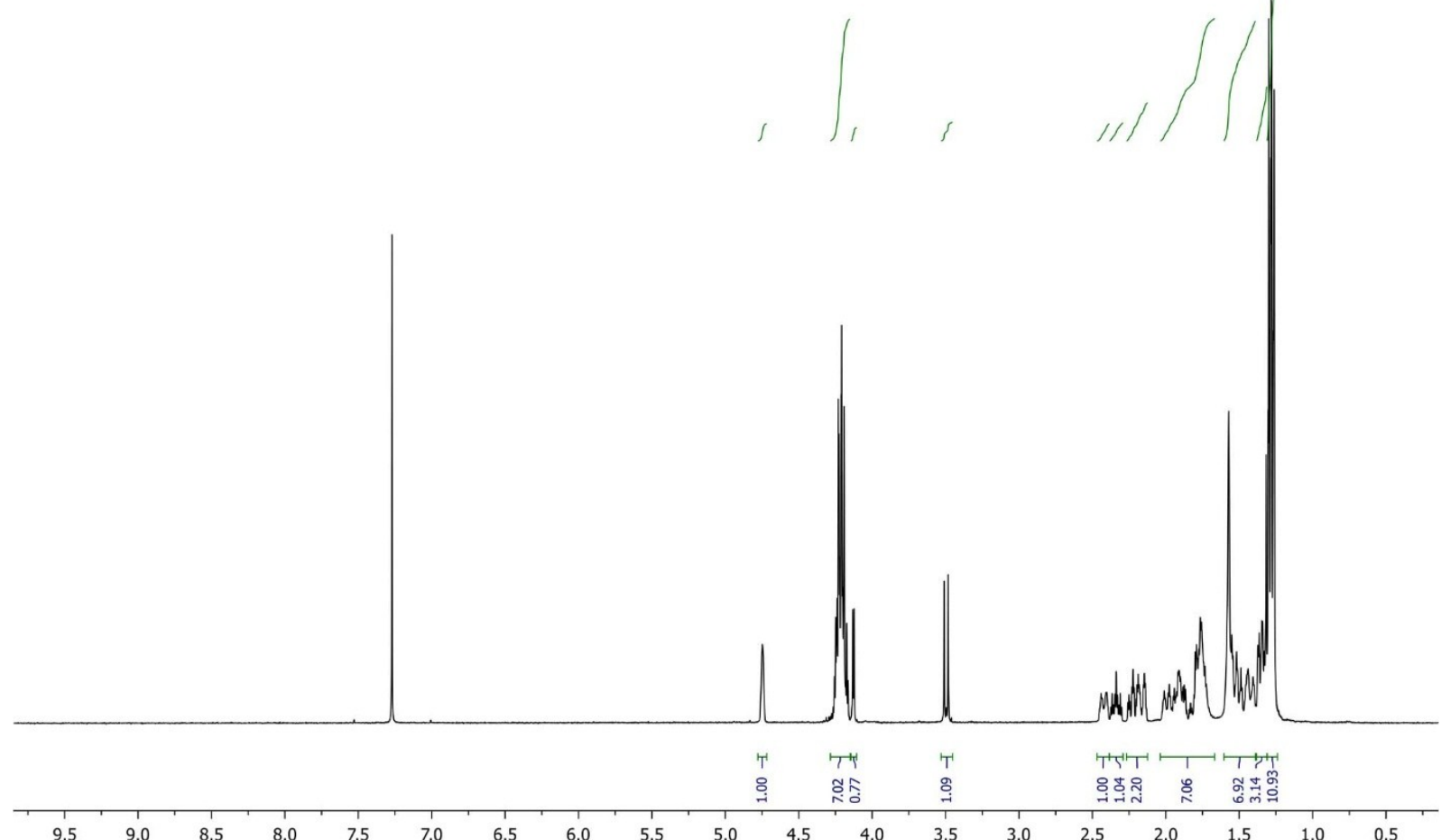

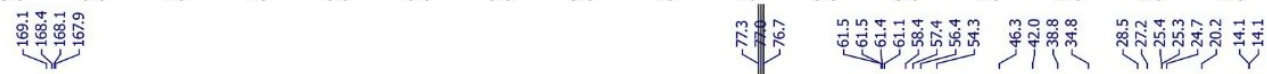

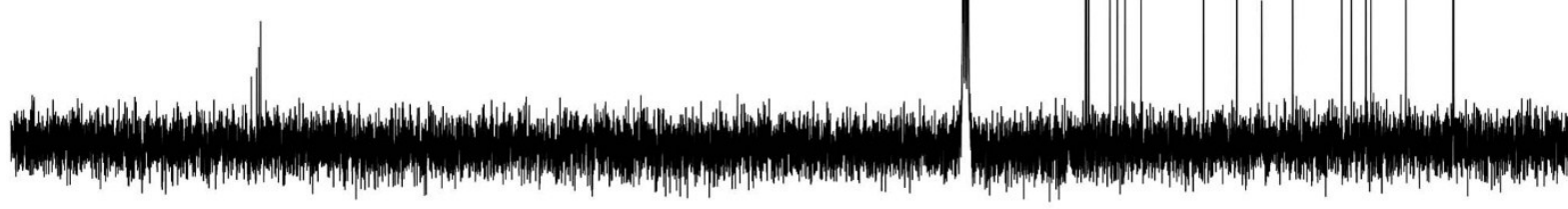

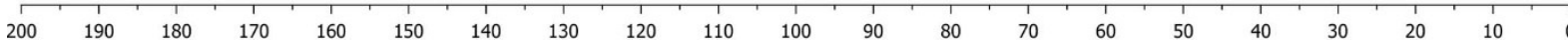


MeO COOEt

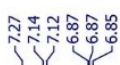

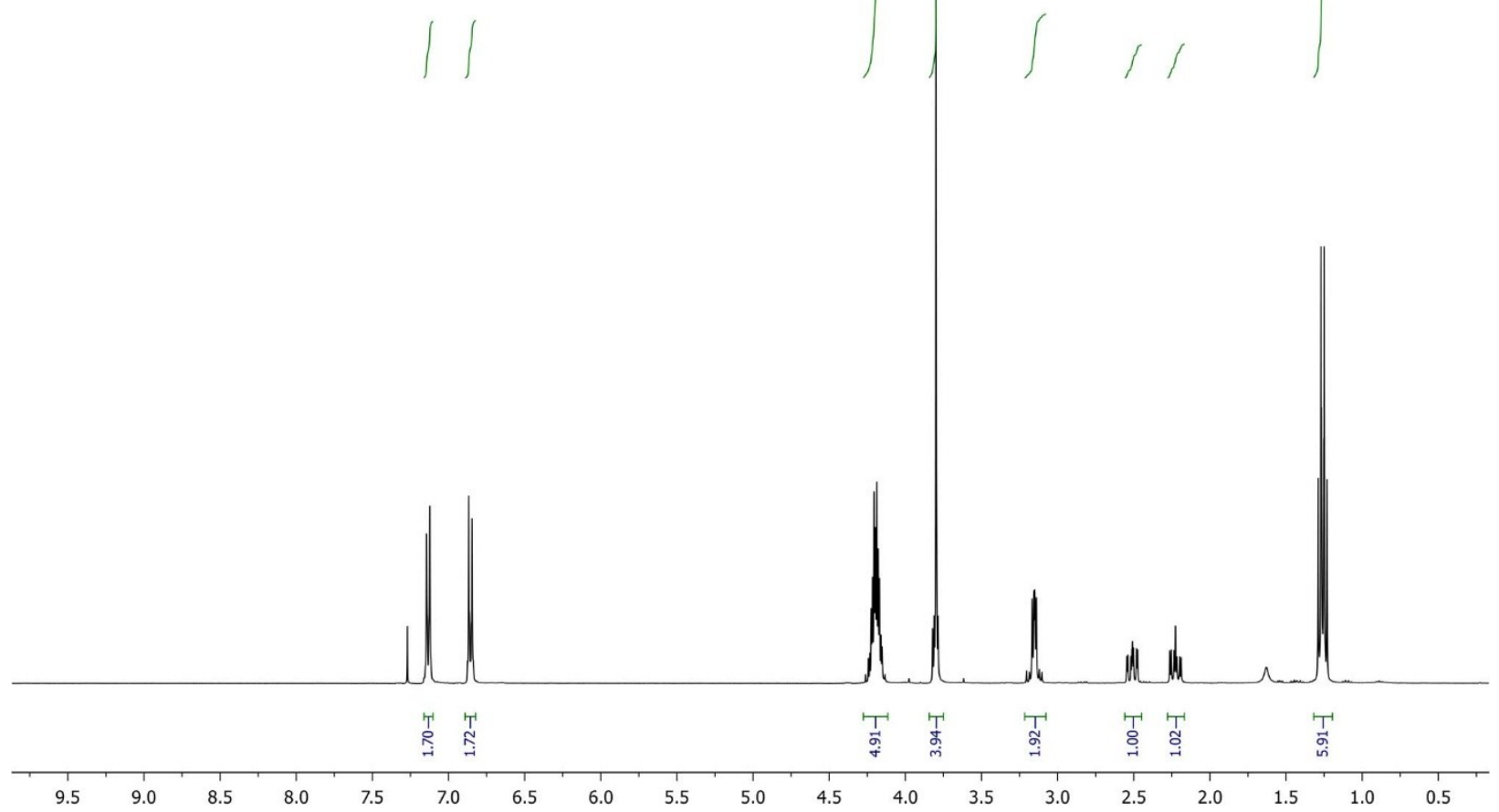

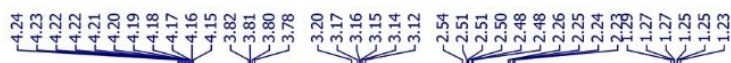

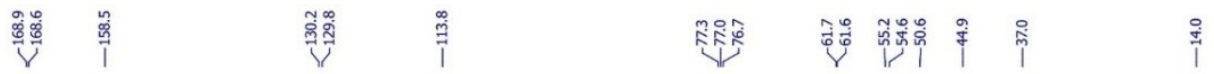

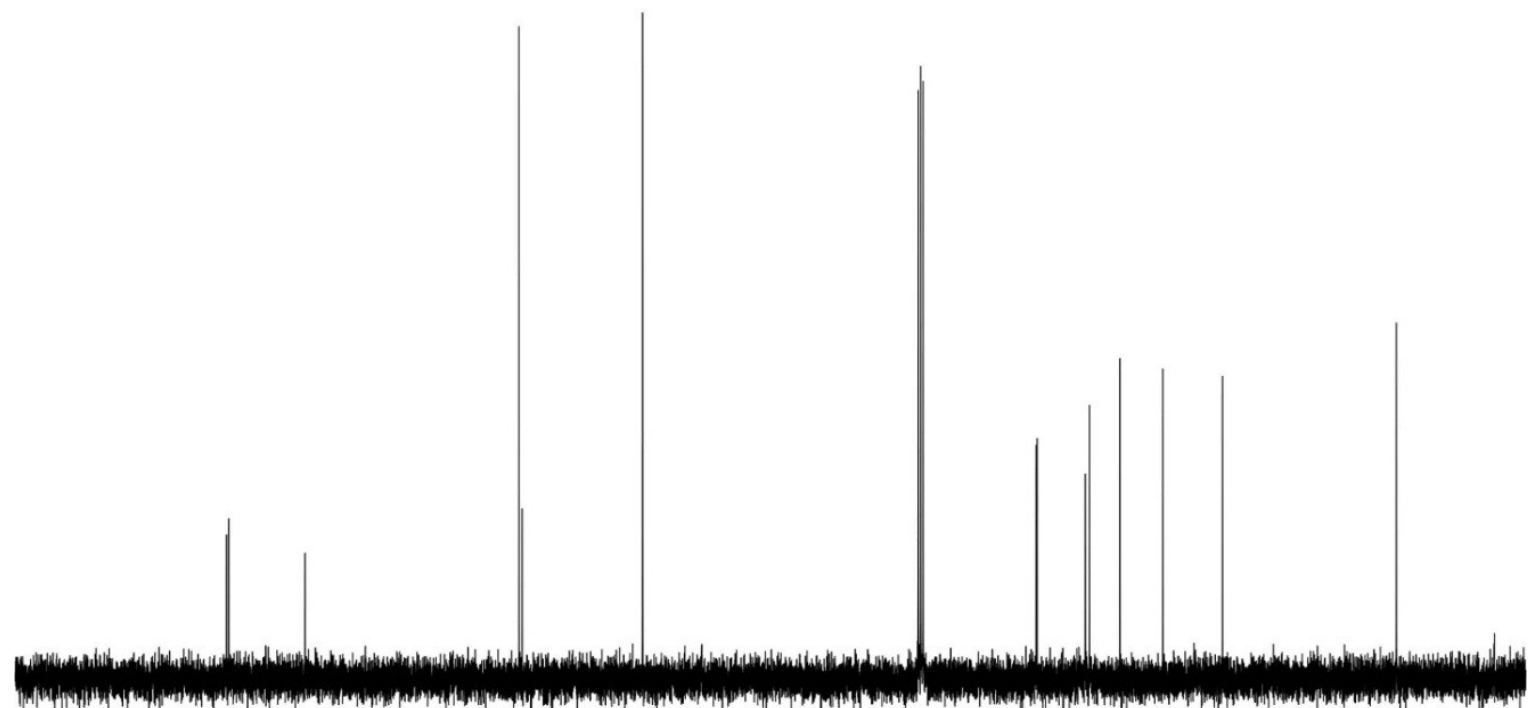

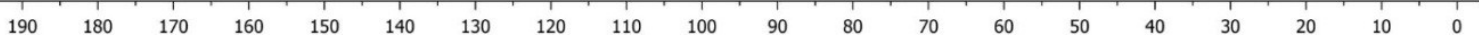




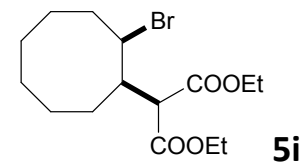

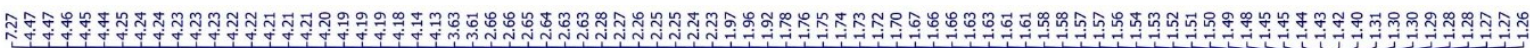
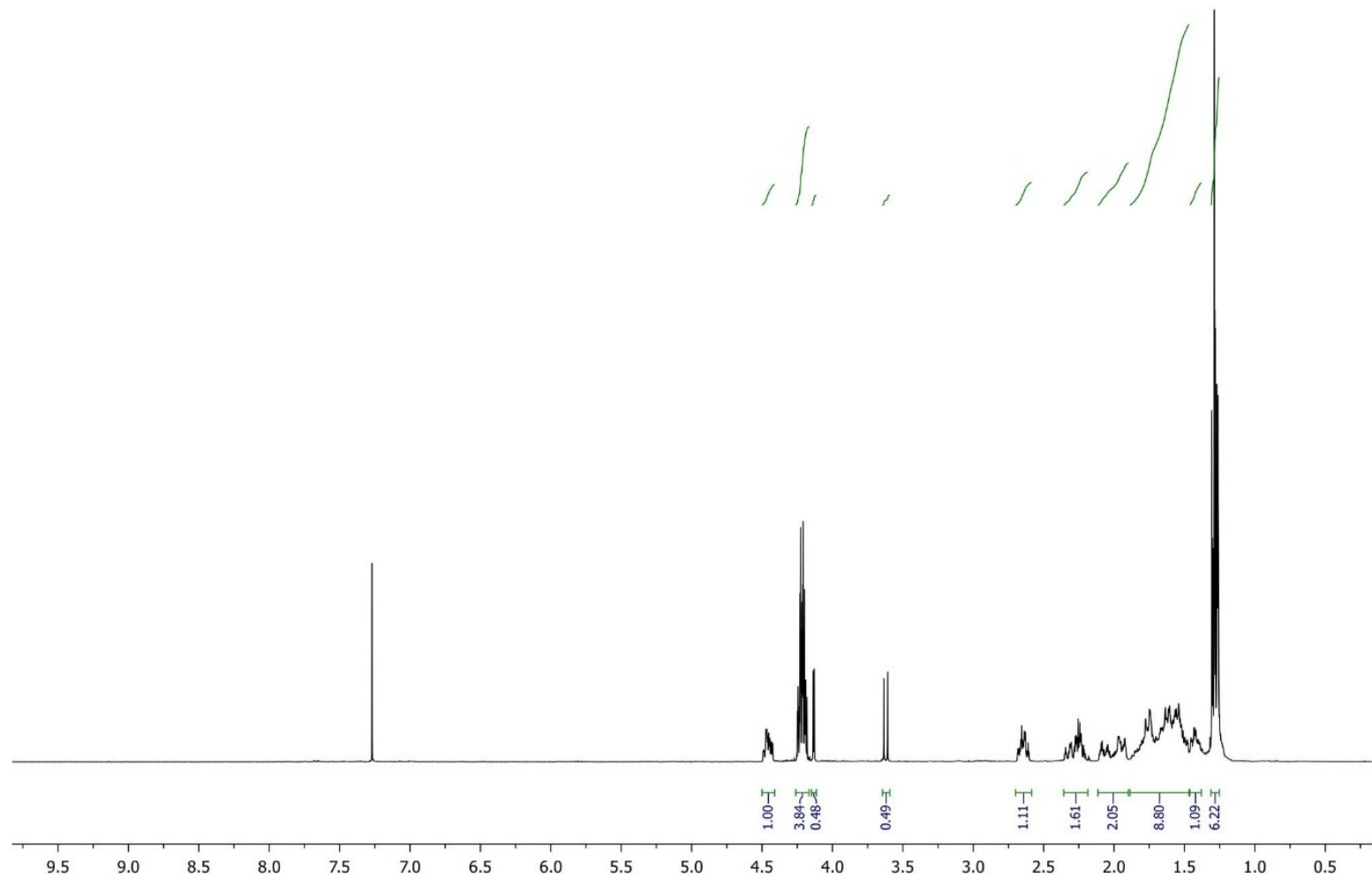

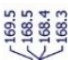

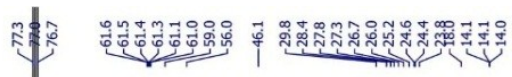

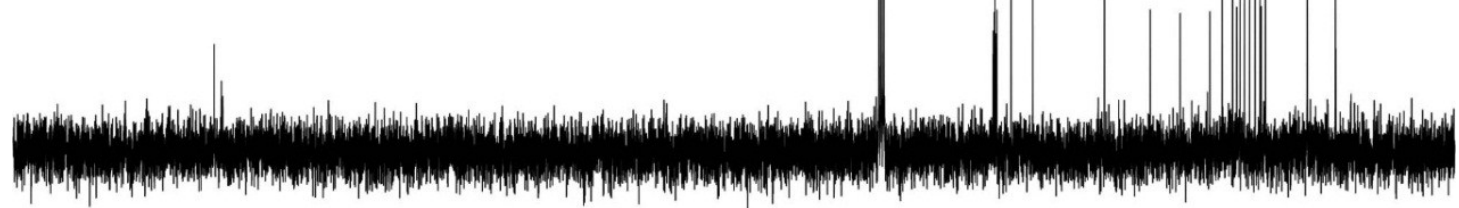

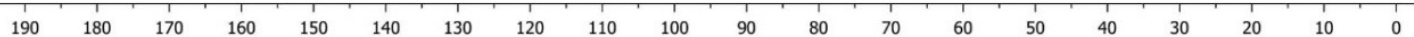



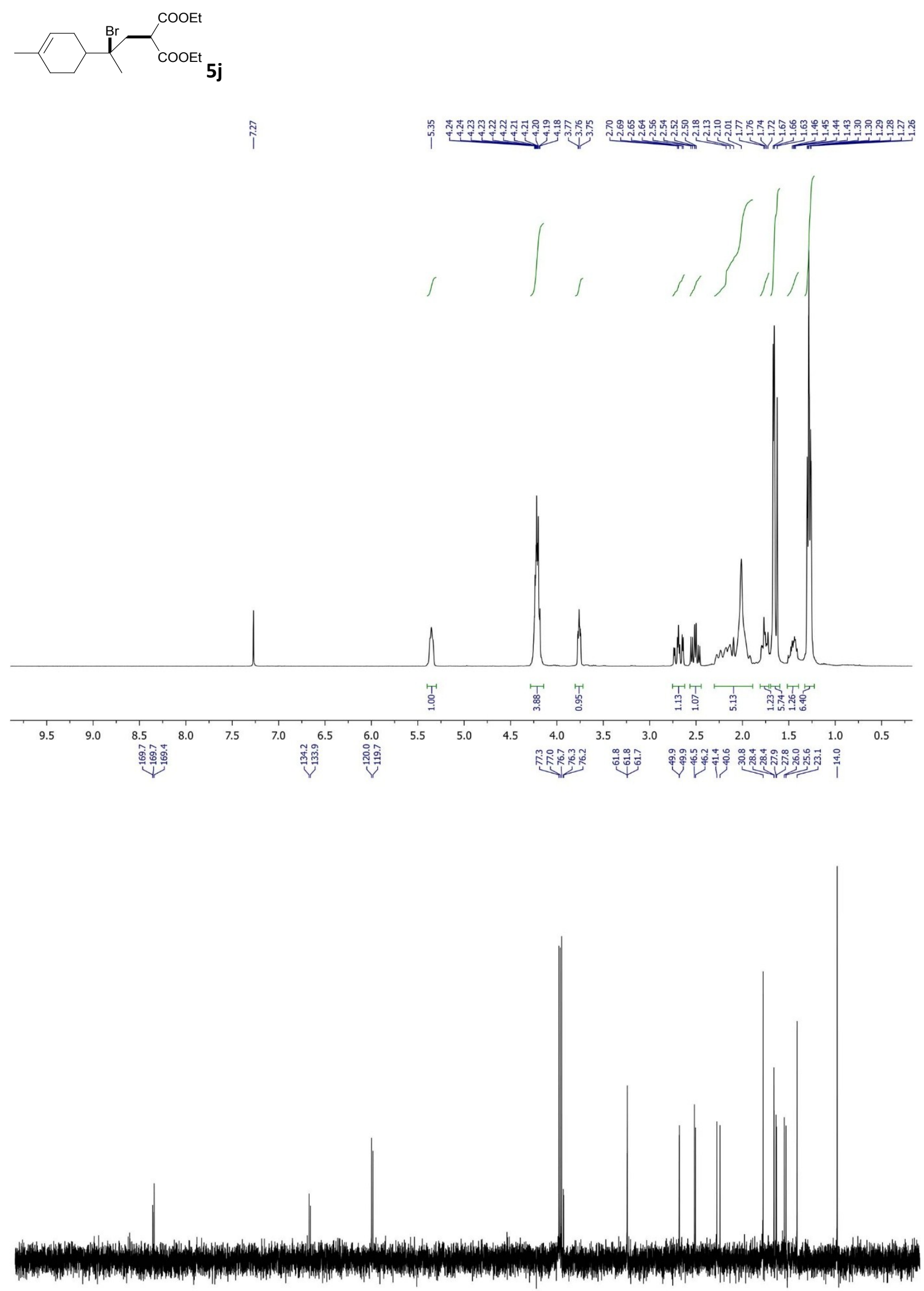

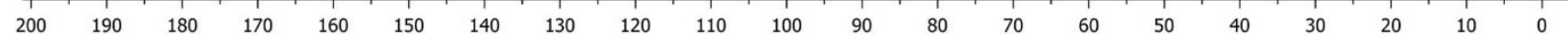


COOEt

5k

în $\quad$ i

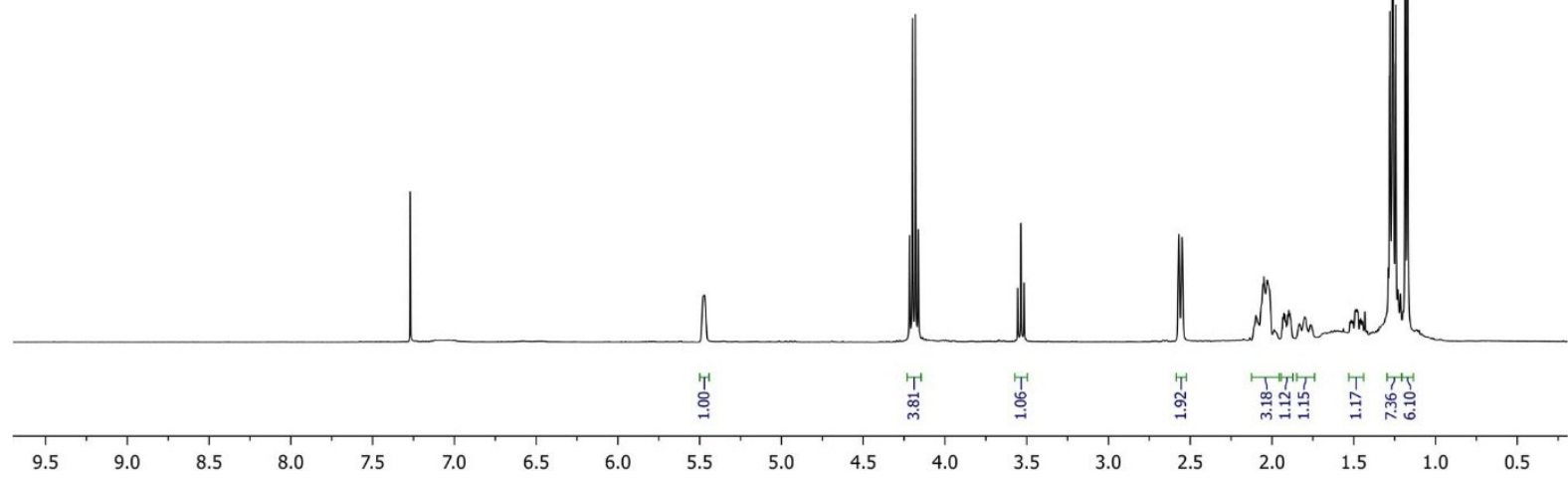

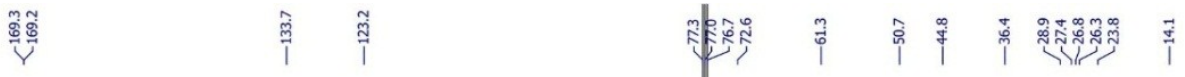

$\begin{array}{llllllllllllllllllll}190 & 180 & 170 & 160 & 150 & 140 & 130 & 120 & 110 & 100 & 90 & 80 & 70 & 60 & 50 & 40 & 30 & 20 & 10 & 0\end{array}$ 


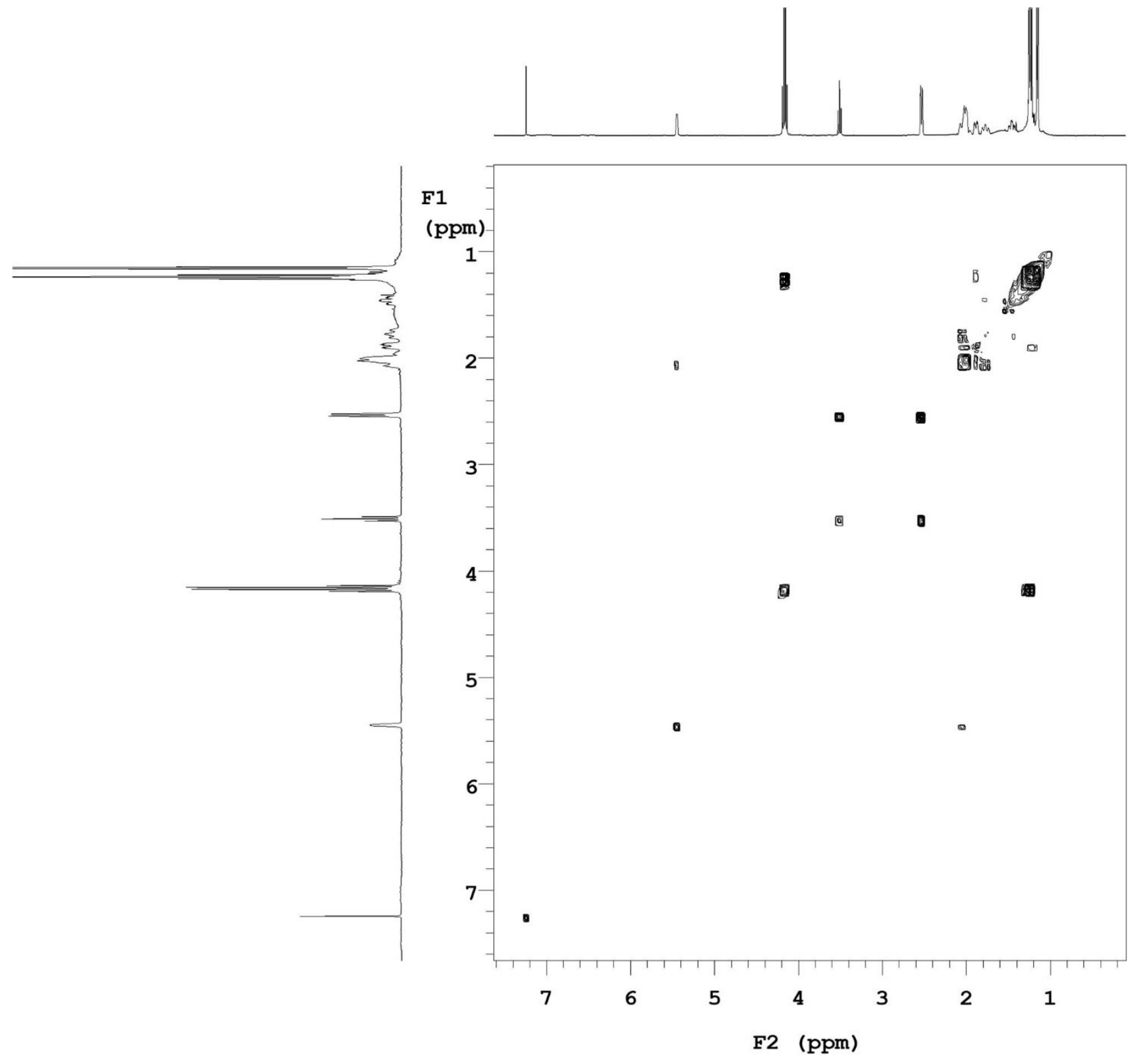

g-COSY of $\mathbf{5 k}$. 


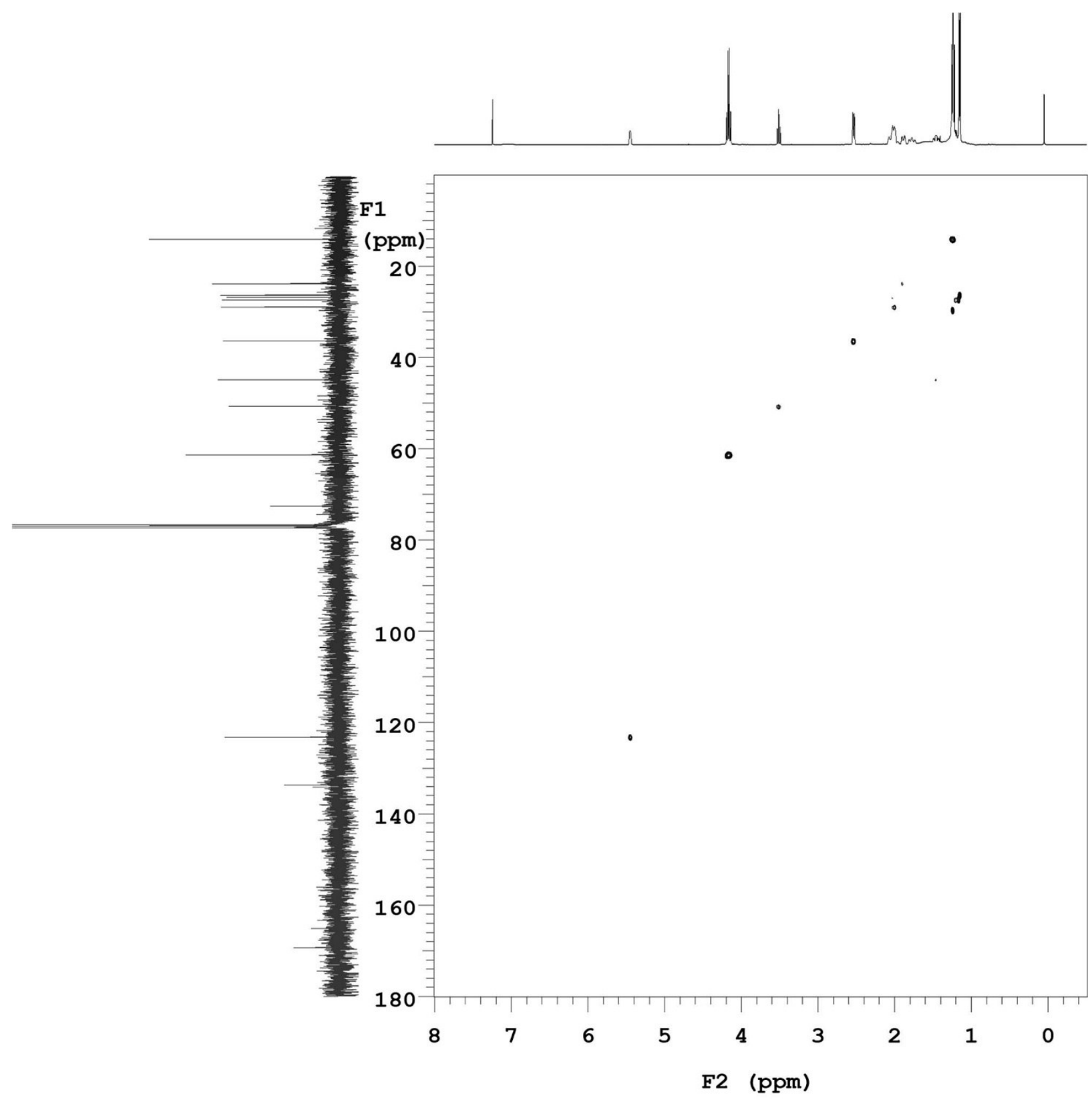

g-HSQC of 5k. Positive and negative contours are shown. 


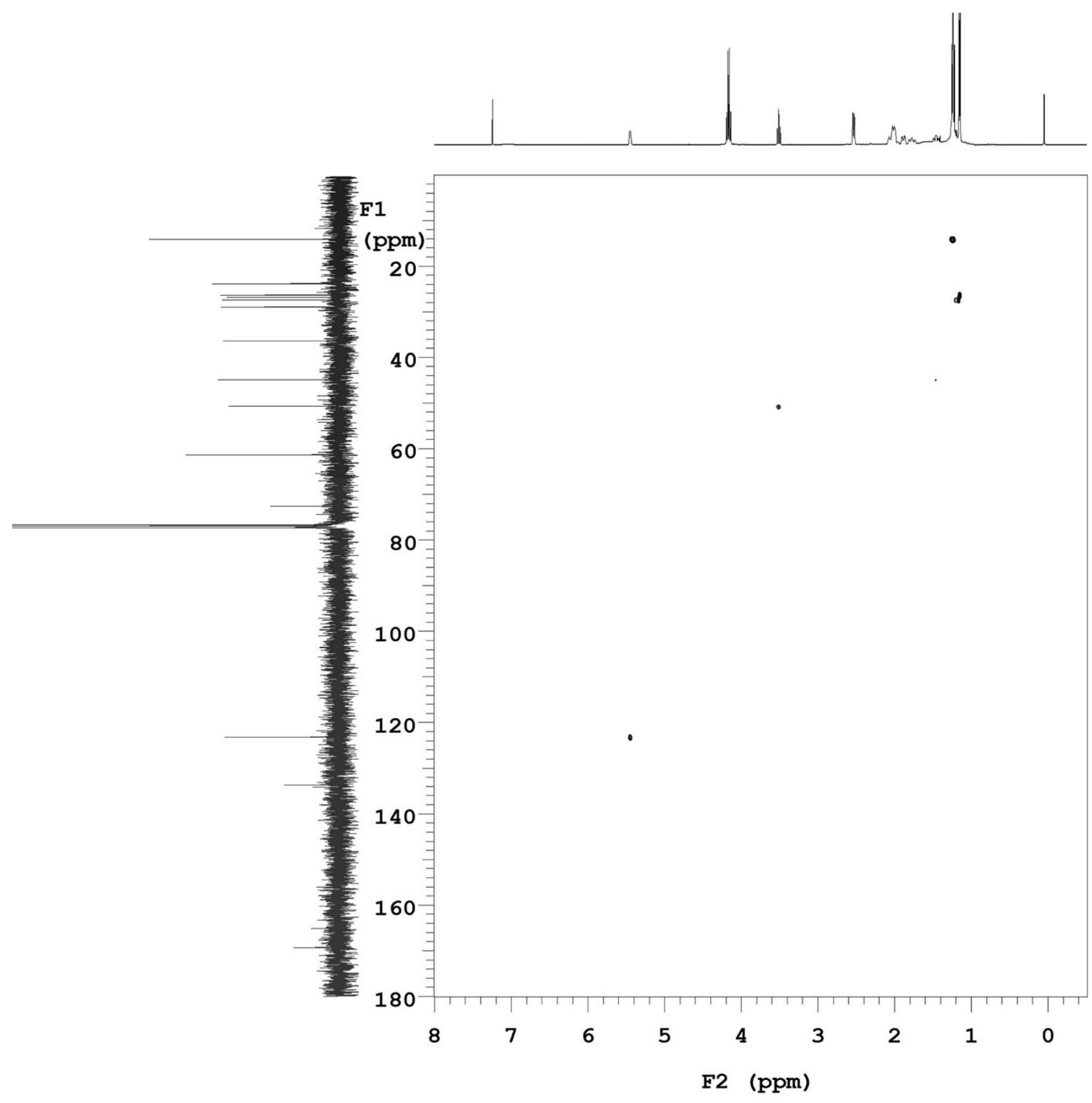

g-HSQC of 5k. Positive contours $\left(\mathrm{CH}\right.$ and $\left.\mathrm{CH}_{3}\right)$ are shown. 


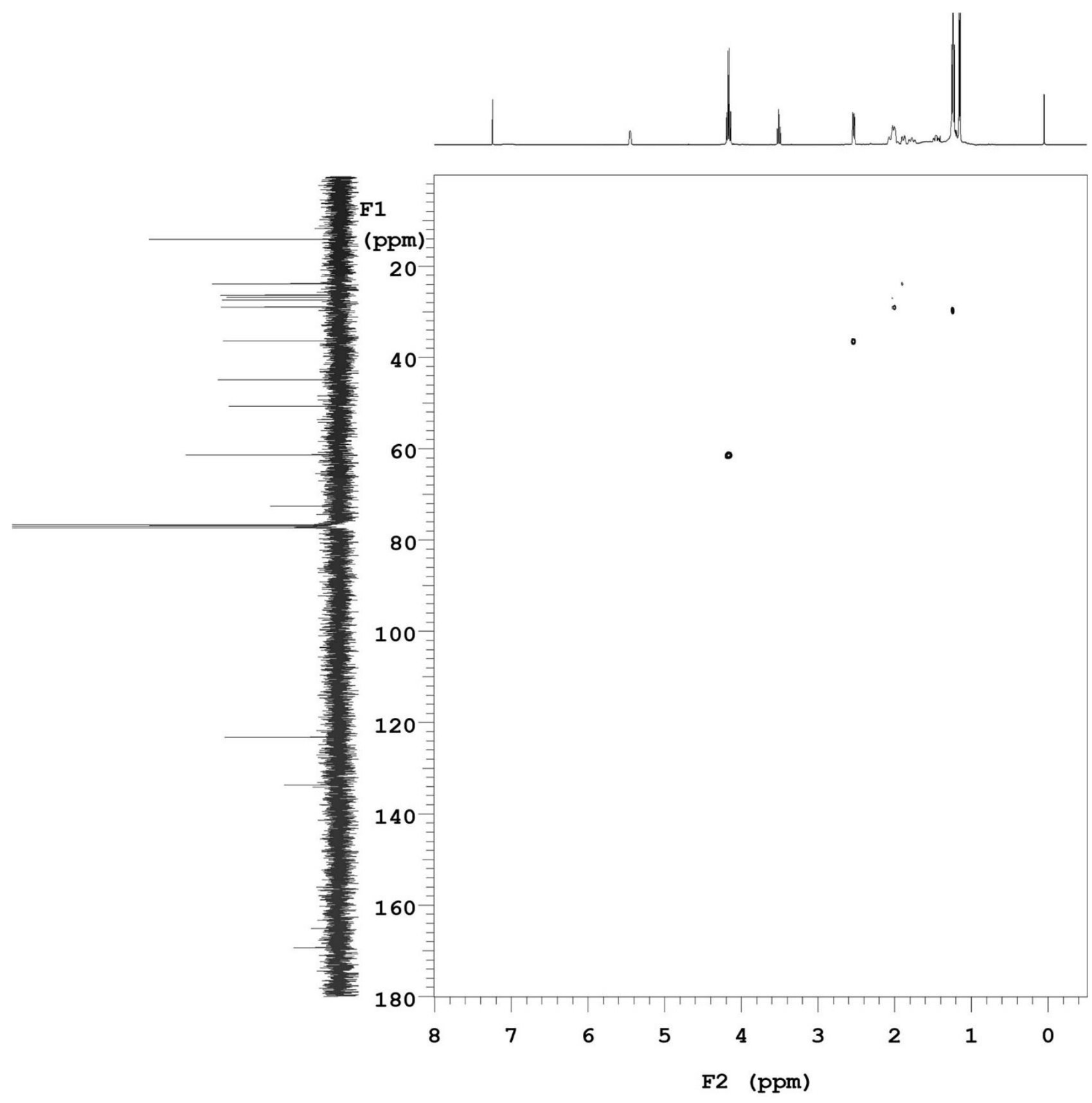

g-HSQC of $\mathbf{5 k}$. Negative contours $\left(\mathrm{CH}_{2}\right)$ are shown. 
$\overbrace{\text { COOEt }}^{\mathrm{COOEt}}$
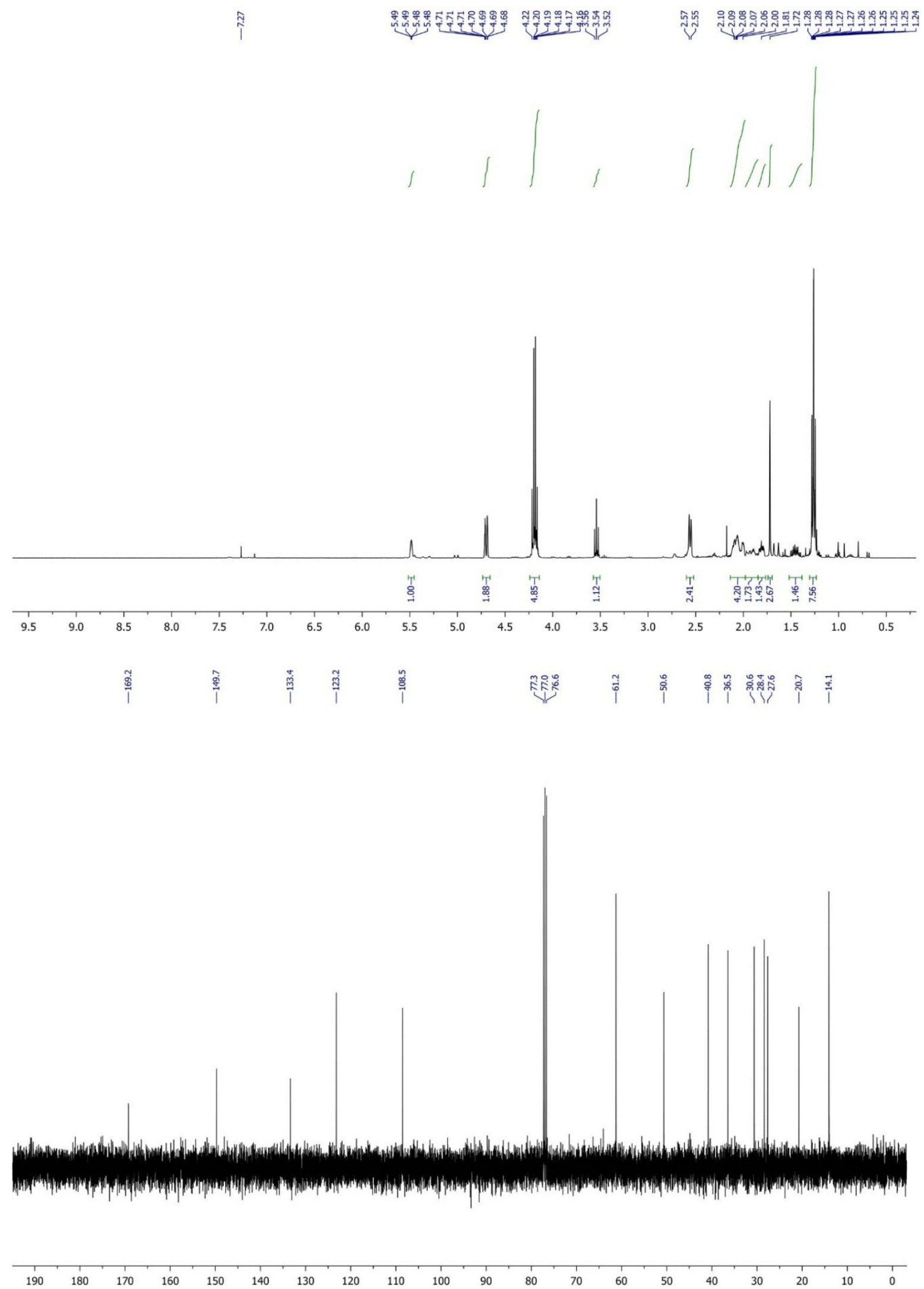


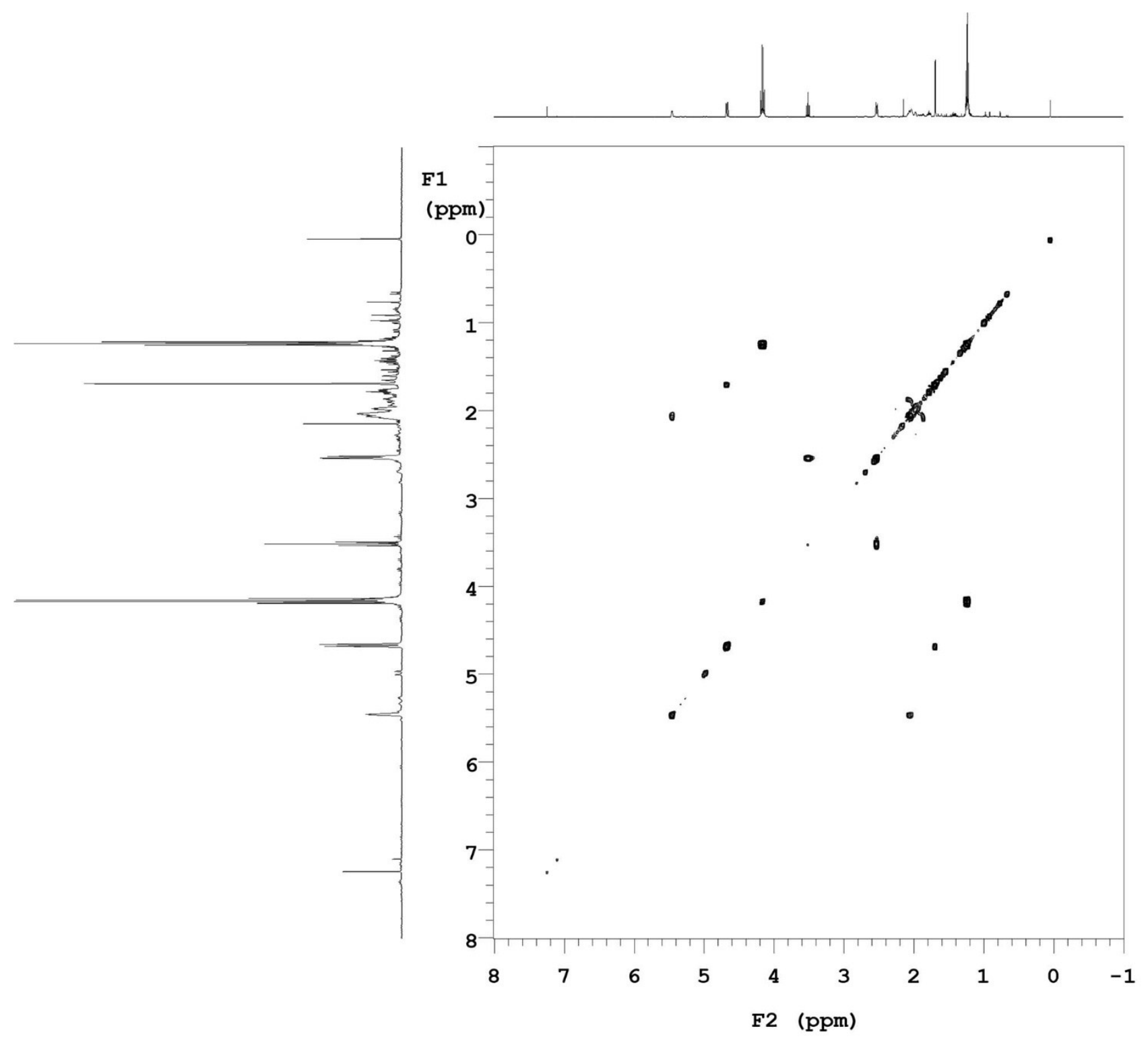

g-COSY of $\mathbf{5} \mathbf{k}^{\prime}$. 


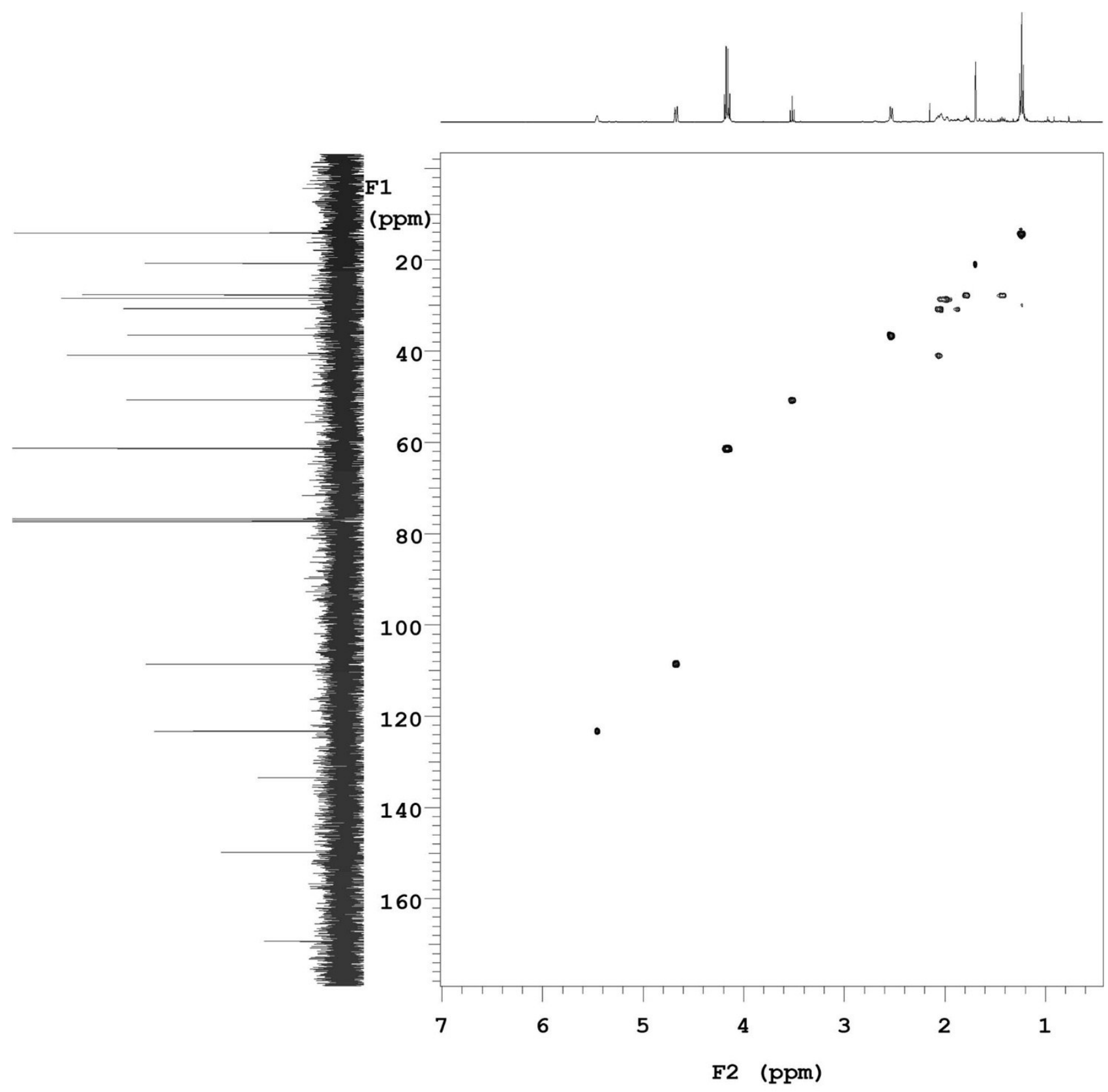

g-HSQC of $\mathbf{5} \mathbf{k}^{\prime}$. Positive and negative contours are shown. 


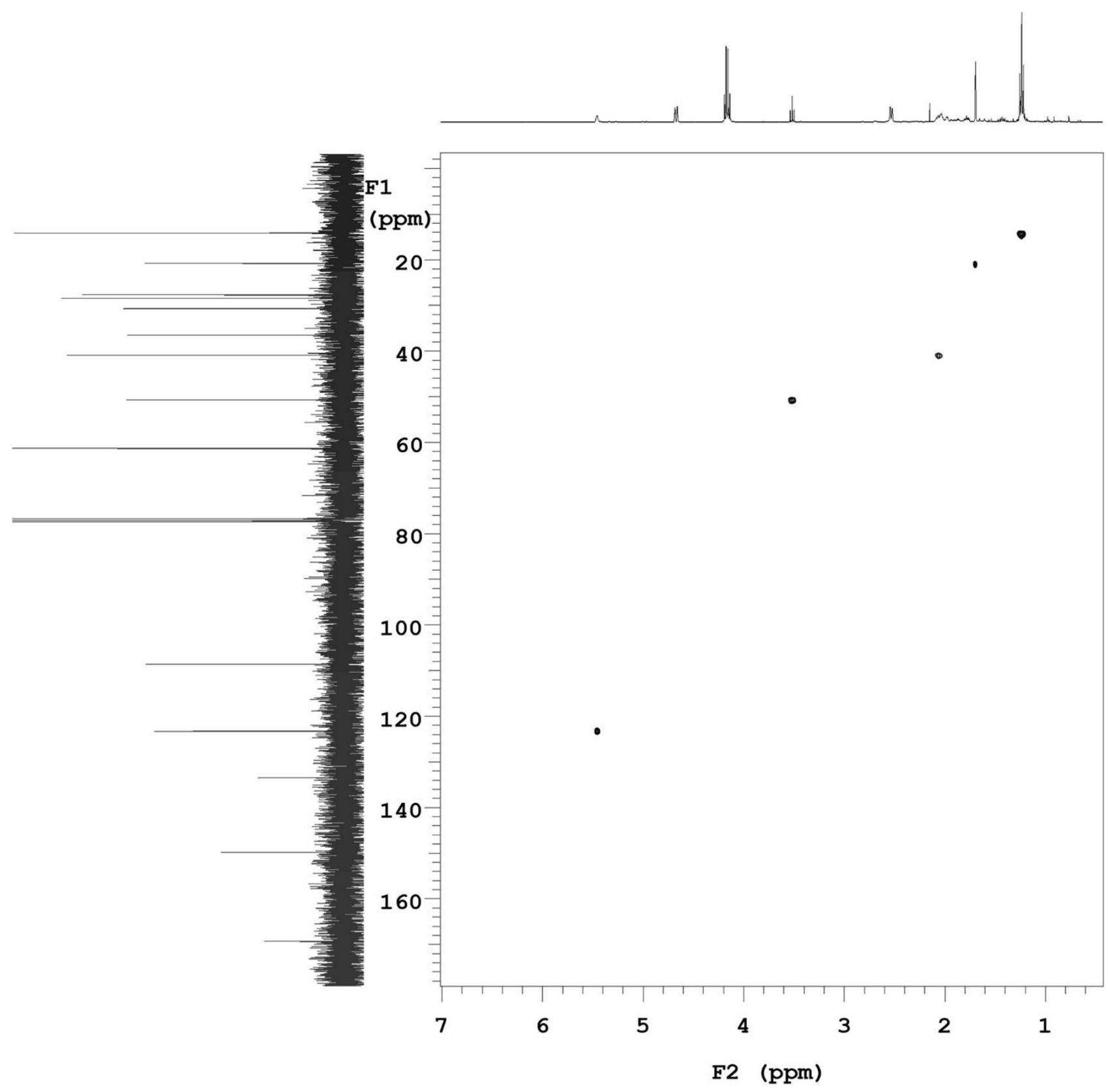

g-HSQC of $\mathbf{5} \mathbf{k}^{\prime}$. Positive contours $\left(\mathrm{CH}\right.$ and $\left.\mathrm{CH}_{3}\right)$ are shown. 


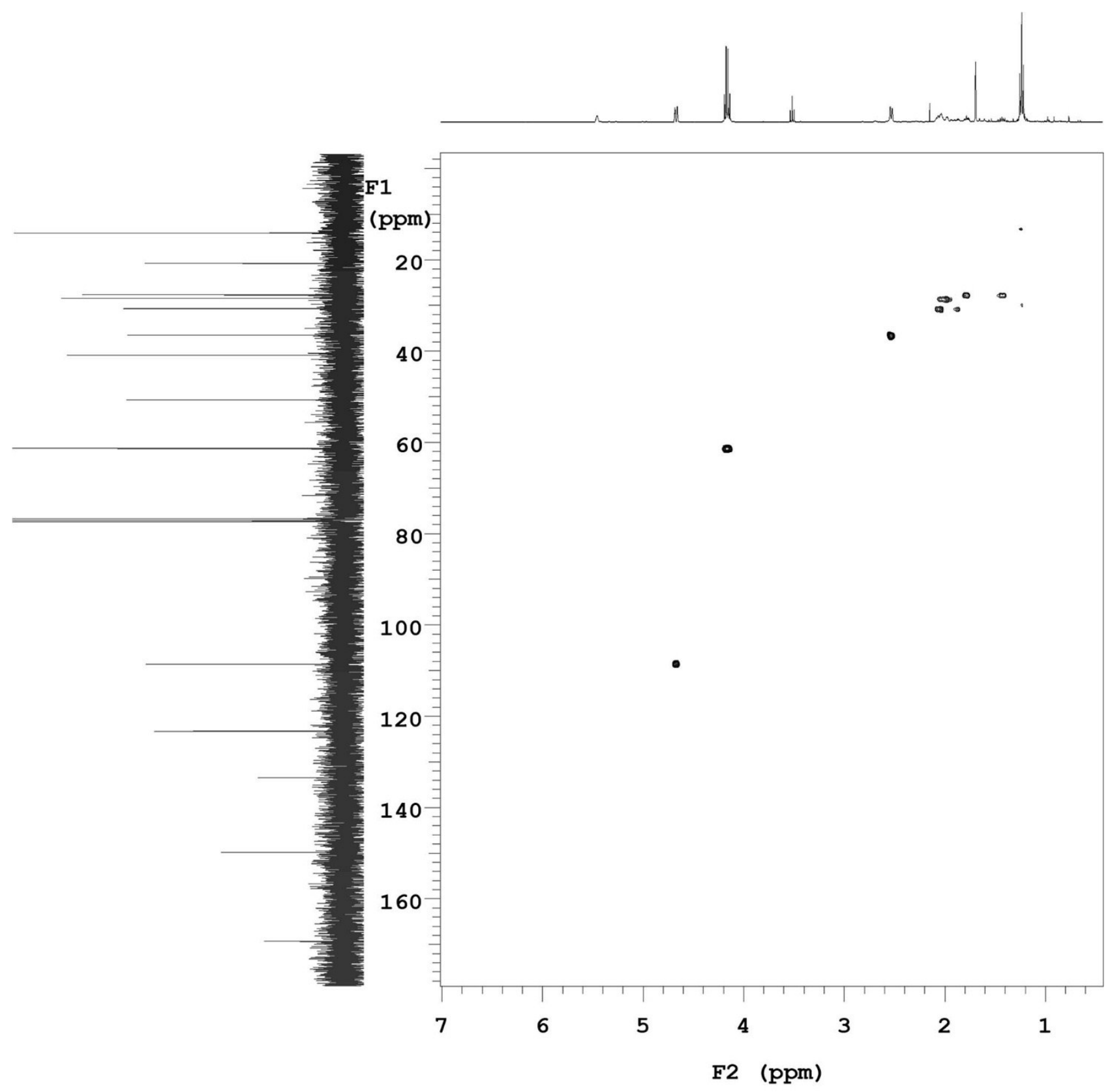

g-HSQC of 5k'. Negative contours $\left(\mathrm{CH}_{2}\right)$ are shown. 